\title{
Intermolecular cyclopropanation of styrenes using iodine and visible light via carbon-iodine bond cleavage
}

Kaoru Usami, Yoshitomo Nagasawa, Eiji Yamaguchi, Norihiro Tada, Akichika Itoh *

Gifu Pharmaceutical University, 1-25-4 Daigaku-nishi, Gifu 501-1196, Japan

E-mail: itoha@gifu-pu.ac.jp

Table of Contents

1. General Information

SI-2

2. Direct cyclopropanation of styrenes and active methylene compounds

SI-2

3. Reference

SI-10

4. $\quad{ }^{1} \mathrm{H}$ and ${ }^{13} \mathrm{C}$ spectra

SI-11 


\section{General Information.}

Unless otherwise noted, all reactants or reagents including dry solvents were obtained from commercial suppliers and used as received. Flash column chromatography was performed with Kanto silica gel 60N (Spherical, Neutral, 40-50 mm). Analytical thin-layer chromatography (TLC) was carried out using $0.25 \mathrm{~mm}$ commercial silica gel plates (Merck silica gel $60 \mathrm{~F}_{254}$ ). The developed chromatogram was analyzed by UV lamp (254 nm). ${ }^{1} \mathrm{H}$ NMR and ${ }^{13} \mathrm{C}$ NMR spectra were obtained on a JEOL ECA 500 (500 MHz for ${ }^{1} \mathrm{H}$ NMR and $125 \mathrm{MHz}$ for ${ }^{13} \mathrm{C}$ NMR) and JEOL AL $400\left(400 \mathrm{MHz}\right.$ for ${ }^{1} \mathrm{H} \mathrm{NMR}$ and $100 \mathrm{MHz}$ for $\left.{ }^{13} \mathrm{C} \mathrm{NMR}\right)$. Chemical shifts $(\delta)$ are expressed in parts per million and are internally referenced [0.00 ppm (tetramethylsilane) for ${ }^{1} \mathrm{H}$ NMR and $77.0 \mathrm{ppm}\left(\mathrm{CDCl}_{3}\right)$ for ${ }^{13} \mathrm{C}$ NMR. Infrared spectra were taken on a Perkin Elmer Spectrum $100 \mathrm{FTIR}$ and are reported in reciprocal centimeters $\left(\mathrm{cm}^{-1}\right)$. High-resolution mass spectra (HRMS) were obtained on a JEOL JMS-T100TD and JEOL JMS-T100GC and are reported as $\mathrm{m} / \mathrm{z}$ (relative intensity). Melting points were measured on a Yanaco Micro Melting Point Apparatus and are uncorrected.

\section{Direct cyclopropanation of styrenes and active methylene compounds}

A solution of styrene $(0.3 \mathrm{mmol})$, active methylene compound $(0.3 \mathrm{mmol}), \mathrm{I}_{2}(76.1 \mathrm{mg}, 0.3 \mathrm{mmol})$ and $\mathrm{K}_{2} \mathrm{CO}_{3}(41.5 \mathrm{mg}, 0.3 \mathrm{mmol})$ in EtOAc $(5 \mathrm{~mL})$ was irradiated with fluorescent lamp $(23 \mathrm{~W} \mathrm{x} 4)$ at approximately $6 \mathrm{~cm}$ away from the light source under an argon atmosphere and stirred for 20 h. The reaction mixture was washed with aq. $\mathrm{Na}_{2} \mathrm{~S}_{2} \mathrm{O}_{3}$, extracted with $\mathrm{Et}_{2} \mathrm{O}$, and then the organic layer was dried over $\mathrm{MgSO}_{4}$ and concentrated in vacuo. Purification of the crude product by flash chromatography on silica gel provided desired product.

\section{Ethyl 1-cyano-2-phenylcyclopropanecarboxylate (3a) (Table 1) ${ }^{1}$}

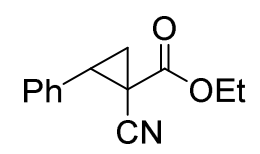

The product was isolated as a yellow oil $\left(55.5 \mathrm{mg}, 86 \%\right.$ yield). Diastereomeric ratio (6:1) was determined by ${ }^{1} \mathrm{H}$ NMR analysis of the crude mixture.

$\mathrm{R}_{f}=0.54(20 \%$ EtOAc in $n$-Hexane $)$.

${ }^{1} \mathrm{H}$ NMR (400 MHz, $\left.\mathrm{CDCl}_{3}\right) \delta$ Major 7.28-7.10 (m, 5H), 4.34-4.25 (m, 2H), $3.14(\mathrm{t}, J=8.7 \mathrm{~Hz}, 1 \mathrm{H}), 2.14(\mathrm{dd}, J=5.3 \mathrm{~Hz}, J=9.2 \mathrm{~Hz}, 1 \mathrm{H}), 2.08$ $(\mathrm{dd}, J=5.3 \mathrm{~Hz}, J=8.2 \mathrm{~Hz}, 1 \mathrm{H}), 1.35(\mathrm{t}, J=7.3 \mathrm{~Hz}, 3 \mathrm{H})$.

Minor 7.28- $7.10(\mathrm{~m}, 5 \mathrm{H}), 4.02-3.96(\mathrm{~m}, 2 \mathrm{H}), 3.26(\mathrm{t}, J=9.2 \mathrm{~Hz}, 1 \mathrm{H}), 2.36-2.31(\mathrm{~m}, 1 \mathrm{H}), 2.00(\mathrm{dd}, J=5.3 \mathrm{~Hz}, J=9.2 \mathrm{~Hz}, 1 \mathrm{H}), 1.05(\mathrm{t}, J=7.2 \mathrm{~Hz}$, $3 \mathrm{H})$.

${ }^{13} \mathrm{C}$ NMR $\left(100 \mathrm{MHz}, \mathrm{CDCl}_{3}\right) \delta 167.3,164.1,138.4,138.0,129.8,129.7,129.1,129.0,128.9,128.1,119.1,116.4,62.9,62.4,36.3,35.4,22.7,22.6$, $21.1,19.9,14.0,13.8$.

Ethyl 1-cyano-2-(4-methoxyphenyl)cyclopropanecarboxylate (3b) (Table 2)

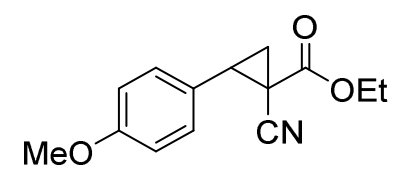

The product was isolated as a yellow oil ( $15.5 \mathrm{mg}, 21 \%$ yield). Diastereomeric ratio (4:1) was determined by ${ }^{1} \mathrm{H}$ NMR analysis of the crude mixture. 
$\mathrm{R}_{f}=0.35(20 \%$ EtOAc in $n$-Hexane)

${ }^{1} \mathrm{H}$ NMR (400 MHz, $\left.\mathrm{CDCl}_{3}\right) \delta$ Major 7.26-7.20 (m, $\left.2 \mathrm{H}\right), 6.91-6.90(\mathrm{~m}, 2 \mathrm{H}), 4.33-4.28(\mathrm{~m}, 2 \mathrm{H}), 3.81(\mathrm{~s}, 3 \mathrm{H}), 3.13(\mathrm{t}, J=8.7 \mathrm{~Hz}, 1 \mathrm{H}), 2.14(\mathrm{dd}$, $J=5.3 \mathrm{~Hz}, J=9.2 \mathrm{~Hz}, 1 \mathrm{H}), 2.06(\mathrm{dd}, J=5.3 \mathrm{~Hz}, J=8.2 \mathrm{~Hz}, 1 \mathrm{H}), 1.36(\mathrm{t}, J=7.2 \mathrm{~Hz}, 3 \mathrm{H})$.

Minor 7.26-7.20 (m, $2 \mathrm{H}), 6.85-6.83(\mathrm{~m}, 2 \mathrm{H}), 4.01-4.00(\mathrm{~m}, 2 \mathrm{H}), 3.79(\mathrm{~s}, 3 \mathrm{H}), 3.25$ (t, $J=9.2 \mathrm{~Hz}, 1 \mathrm{H}), 2.34(\mathrm{dd}, J=5.3 \mathrm{~Hz}, J=8.7 \mathrm{~Hz}, 1 \mathrm{H})$, $2.00(\mathrm{dd}, J=5.3 \mathrm{~Hz}, J=9.6 \mathrm{~Hz}, 1 \mathrm{H}), 1.07(\mathrm{t}, J=7.2 \mathrm{~Hz}, 3 \mathrm{H})$.

${ }^{13} \mathrm{C}$ NMR $\left(100 \mathrm{MHz}, \mathrm{CDCl}_{3}\right) \delta 167.4,159.7,132.8,130.4,129.5,129.2,127.1,124.8,116.6,114.2,113.9,113.7,63.0,62.5,55.3,55.1,36.2,35.2$, $29.7,29.3,22.9,20.0,14.1,13.9$.

IR (ATR) 2937, 2839, 2244, 1729, 1249, $1098 \mathrm{~cm}^{-1}$.

HRMS (DART) Found 246.1140, Calcd. for $\mathrm{C}_{14} \mathrm{H}_{16} \mathrm{NO}_{3},[\mathrm{M}+\mathrm{H}]^{+} 246.1130$.

\section{Ethyl 1-cyano-2-(4-tert-butylphenyl)cyclopropanecarboxylate (3c) (Table 2)}

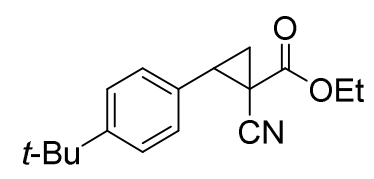

The product was isolated as a yellow viscous oil (67.6 mg, 83\% yield). Diastereomeric ratio (5:1) was determined by ${ }^{1} \mathrm{H}$ NMR analysis of the crude mixture.

$\mathrm{R}_{f}=0.60(20 \%$ EtOAc in $n$-Hexane).

${ }^{1} \mathrm{H}$ NMR (500 MHz, $\mathrm{CDCl}_{3}$ ) $\delta$ Major 7.40-7.20 (m, $\left.4 \mathrm{H}\right), 4.29(\mathrm{q}, J=6.9 \mathrm{~Hz}, 2 \mathrm{H}), 3.13(\mathrm{t}, J=9.2 \mathrm{~Hz}, 1 \mathrm{H}), 2.14(\mathrm{dd}, J=5.2 \mathrm{~Hz}, J=9.8 \mathrm{~Hz}, 1 \mathrm{H})$, $2.07(\mathrm{dd}, J=5.2 \mathrm{~Hz}, J=8.6 \mathrm{~Hz}, 1 \mathrm{H}), 1.35$ (t, $J=6.9 \mathrm{~Hz}, 3 \mathrm{H}), 1.31(\mathrm{~s}, 9 \mathrm{H})$.

Minor 7.40-7.20 (m, $4 \mathrm{H}), 3.96$ (q, $J=6.9 \mathrm{~Hz}, 2 \mathrm{H}), 3.25$ (t, $J=9.2 \mathrm{~Hz}, 1 \mathrm{H}), 2.35(\mathrm{dd}, J=5.7 \mathrm{~Hz}, J=9.2 \mathrm{~Hz}, 1 \mathrm{H}), 1.99(\mathrm{dd}, J=5.7 \mathrm{~Hz}, J=9.2$ $\mathrm{Hz}, 1 \mathrm{H}), 1.28(\mathrm{~s}, 9 \mathrm{H}), 0.95(\mathrm{t}, J=6.9 \mathrm{~Hz}, 3 \mathrm{H})$.

${ }^{13} \mathrm{C}$ NMR $\left(125 \mathrm{MHz}, \mathrm{CDCl}_{3}\right) \delta 167.3,164.1,151.4,151.2,129.7,128.8,127.8,125.7,125.6,125.3,125.1,119.1,116.4,62.9,62.3,36.1,35.2$, $34.5,31.1,29.6,22.8,20.9,19.8,14.0,13.6$.

IR (ATR) 2963, 2870, 2244, 1731, $1264 \mathrm{~cm}^{-1}$.

HRMS (DART) Found 272.1677, Calcd. for $\mathrm{C}_{17} \mathrm{H}_{22} \mathrm{NO}_{2},[\mathrm{M}+\mathrm{H}]^{+} 272.1651$.

\section{Ethyl 1-cyano-2-(4-methylphenyl)cyclopropanecarboxylate (3d) (Table 2)}

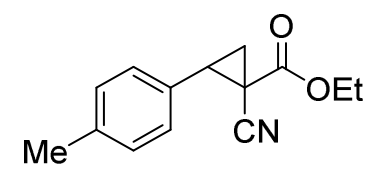

The product was isolated as a yellow oil $\left(54.3 \mathrm{mg}, 79 \%\right.$ yield). Diastereomeric ratio (5:1) was determined by ${ }^{1} \mathrm{H}$ NMR analysis of the crude mixture.

$\mathrm{R}_{f}=0.45(20 \%$ EtOAc in $n$-Hexane).

${ }^{1} \mathrm{H}$ NMR (400 MHz, $\mathrm{CDCl}_{3}$ ) $\delta$ Major 7.25-7.12 (m, $\left.4 \mathrm{H}\right), 4.29(\mathrm{q}, J=6.8 \mathrm{~Hz}, 2 \mathrm{H}), 3.14(\mathrm{dd}, J=8.2 \mathrm{~Hz}, J=9.2 \mathrm{~Hz}, 1 \mathrm{H}), 2.34(\mathrm{~s}, 3 \mathrm{H}), 2.14(\mathrm{dd}, J$ $=5.3 \mathrm{~Hz}, J=9.2 \mathrm{~Hz}, 1 \mathrm{H}), 2.07(\mathrm{dd}, J=5.3 \mathrm{~Hz}, J=8.2 \mathrm{~Hz}, 1 \mathrm{H}), 1.35(\mathrm{t}, J=6.8 \mathrm{~Hz}, 3 \mathrm{H})$ 
Minor 7.25-7.12 (m, $4 \mathrm{H}), 3.99$ (q, $J=7.3 \mathrm{~Hz}, 2 \mathrm{H}), 3.25$ (dd, $J=9.2 \mathrm{~Hz}, J=9.7 \mathrm{~Hz}, 1 \mathrm{H}), 2.31(\mathrm{~s}, 3 \mathrm{H}), 2.18-2.06(\mathrm{~m}, 1 \mathrm{H}), 2.00(\mathrm{dd}, J=5.8 \mathrm{~Hz}$, $J=9.7 \mathrm{~Hz}, 1 \mathrm{H}), 1.05(\mathrm{t}, \mathrm{J}=7.2 \mathrm{~Hz}, 3 \mathrm{H})$.

13C NMR (100 MHz, CDCl3) $\delta 167.3,138.3,129.8,129.4,129.3,129.1,129.0,128.9,128.6,128.1,125.2,116.5,63.0,62.4,36.3,35.7,35.3$, $34.1,22.9,22.7,21.1,20.0,14.0,13.8$.

IR (ATR) 2984, 2924, 2245, 1730, $1263 \mathrm{~cm}^{-1}$.

HRMS (DART) Found 230.1192, Calcd. for $\mathrm{C}_{14} \mathrm{H}_{16} \mathrm{NO}_{2}$, [M+ H] $]^{+} 230.1181$.

\section{Ethyl 1-cyano-2-(2-methylphenyl)cyclopropanecarboxylate (3e) (Table 2)}

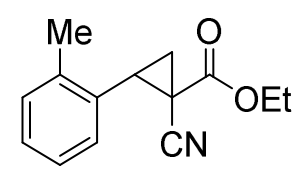

The product was isolated as a yellow oil $\left(50.2 \mathrm{mg}, 73 \%\right.$ yield). Diastereomeric ratio (6:1) was determined by ${ }^{1} \mathrm{H}$ NMR analysis of the crude mixture.

$\mathrm{R}_{f}=0.44(20 \%$ EtOAc in $n$-Hexane)

${ }^{1} \mathrm{H}$ NMR (400 MHz, $\left.\mathrm{CDCl}_{3}\right) \delta$ Major 7.27-7.11 (m, $\left.4 \mathrm{H}\right), 4.34$ (q, $\left.J=7.2 \mathrm{~Hz}, 2 \mathrm{H}\right), 3.08(\mathrm{dd}, J=8.7 \mathrm{~Hz}, J=9.2 \mathrm{~Hz}, 1 \mathrm{H}), 2.35(\mathrm{~s}, 3 \mathrm{H}), 2.18(\mathrm{dd}, J$ $=5.3 \mathrm{~Hz}, J=9.2 \mathrm{~Hz}, 1 \mathrm{H}), 2.14(\mathrm{dd}, J=5.3 \mathrm{~Hz}, J=8.7 \mathrm{~Hz}, 1 \mathrm{H}) 1.36(\mathrm{t}, J=7.2 \mathrm{~Hz}, 3 \mathrm{H})$.

Minor 7.27-7.11 (m, $4 \mathrm{H}), 3.96$ (q, J=7.2 Hz, $2 \mathrm{H}), 3.15$ (m, $1 \mathrm{H}), 2.39$ (s, $3 \mathrm{H}), 2.40-2.36(\mathrm{~m}, 1 \mathrm{H}), 2.07$ (m, $1 \mathrm{H}), 0.99$ (t, $J=7.2 \mathrm{~Hz}, 3 \mathrm{H})$.

${ }^{13} \mathrm{C} \mathrm{NMR}\left(100 \mathrm{MHz}, \mathrm{CDCl}_{3}\right) \delta 167.5,164.2,138.5,138.3,131.7,130.7,130.3,129.8,128.8,128.7,128.3,127.3,126.2,125.6,119.0,116.2,63.0$, $62.4,35.7,34.3,29.6,22.4,21.9,20.6,20.1,19.3,14.1,13.7$.

IR (ATR) 2982, 2924, 2245, 1730, $1263 \mathrm{~cm}^{-1}$.

HRMS (DART) Found 230.1171, Calcd. for $\mathrm{C}_{14} \mathrm{H}_{16} \mathrm{NO}_{2}$, [M+ H] $]^{+} 230.1181$.

\section{Ethyl 1-cyano-2-(3-methylphenyl)cyclopropanecarboxylate (3f) (Table 2)}

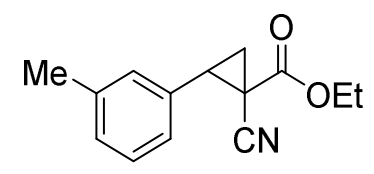

The product was isolated as a brown oil $\left(65.3 \mathrm{mg}, 95 \%\right.$ yield). Diastereomeric ratio $(5: 1)$ was determined by ${ }^{1} \mathrm{H}$ NMR analysis of the crude mixture.

$\mathrm{R}_{f}=0.56(20 \%$ EtOAc in $n$-Hexane $)$.

${ }^{1} \mathrm{H}$ NMR $\left(500 \mathrm{MHz}, \mathrm{CDCl}_{3}\right) \delta$ Major 7.27-7.03 (m, $\left.4 \mathrm{H}\right), 4.28(\mathrm{q}, J=6.9 \mathrm{~Hz}, 2 \mathrm{H}), 3.13(\mathrm{dd}, J=8.6 \mathrm{~Hz}, J=9.2 \mathrm{~Hz}, 1 \mathrm{H}), 2.35(\mathrm{~s}, 3 \mathrm{H}), 2.12(\mathrm{dd}, J$ $=5.2 \mathrm{~Hz}, J=9.2 \mathrm{~Hz}, 1 \mathrm{H}), 2.08(\mathrm{dd}, J=5.2 \mathrm{~Hz}, J=8.6 \mathrm{~Hz}, 1 \mathrm{H}), 1.35(\mathrm{t}, J=6.9 \mathrm{~Hz}, 3 \mathrm{H})$.

Minor 7.27-7.03 (m, $4 \mathrm{H}), 3.96$ (q, $J=6.9 \mathrm{~Hz}, 2 \mathrm{H}), 3.24$ (t, $J=9.2 \mathrm{~Hz}, 1 \mathrm{H}), 2.38-2.33(\mathrm{~m}, 1 \mathrm{H}), 2.31(\mathrm{~s}, 3 \mathrm{H}), 1.98(\mathrm{dd}, J=5.7 \mathrm{~Hz}, J=9.7 \mathrm{~Hz}, 1$ H), $1.02(\mathrm{t}, J=6.9 \mathrm{~Hz}, 3 \mathrm{H})$.

${ }^{13} \mathrm{C} \mathrm{NMR}\left(125 \mathrm{MHz}, \mathrm{CDCl}_{3}\right) \delta 167.2,164.1,138.3,137.8,132.7,131.9,129.9,129.2,129.0,128.9,128.5,128.1,126.1,125.1,119.0,116.3,62.9$, $62.3,36.3,35.3,22.7,22.6,21.2,21.1,20.7,19.8,14.0,13.7$. 
IR (ATR) 2983, 2924, 2245, 1730, $1263 \mathrm{~cm}^{-1}$.

HRMS (DART) Found 230.1178, Calcd. For $\mathrm{C}_{14} \mathrm{H}_{16} \mathrm{NO}_{2},[\mathrm{M}+\mathrm{H}]^{+} 230.1181$.

4

Ethyl 1-cyano-2-(4-chrolophenyl)cyclopropanecarboxylate (3g) (Table 2)

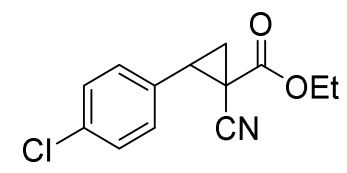

The product was isolated as a yellow oil $\left(67.4 \mathrm{mg}, 90 \%\right.$ yield). Diastereomeric ratio $(5.5: 1)$ was determined by ${ }^{1} \mathrm{H}$ NMR analysis of the crude mixture.

$\mathrm{R}_{f}=0.40(20 \%$ EtOAc in $n$-Hexane).

${ }^{1} \mathrm{H}$ NMR (400 MHz, $\left.\mathrm{CDCl}_{3}\right) \delta$ Major 7.37-7.19 (m, $\left.4 \mathrm{H}\right), 4.31$ (q, $\left.J=6.8 \mathrm{~Hz}, 2 \mathrm{H}\right), 3.13(\mathrm{t}, J=8.7 \mathrm{~Hz}, 1 \mathrm{H}), 2.17(\mathrm{dd}, J=5.3 \mathrm{~Hz}, J=9.2 \mathrm{~Hz}, 1 \mathrm{H})$, $2.06(\mathrm{dd}, J=5.3 \mathrm{~Hz}, J=8.2 \mathrm{~Hz}, 1 \mathrm{H}), 1.36(\mathrm{t}, J=6.8 \mathrm{~Hz}, 3 \mathrm{H})$.

Minor 7.37-7.19 (m, $4 \mathrm{H}), 4.01$ (q, $J=7.2 \mathrm{~Hz}, 2 \mathrm{H}), 3.24(\mathrm{t}, J=9.2 \mathrm{~Hz}, 1 \mathrm{H}), 2.33(\mathrm{dd}, J=5.3 \mathrm{~Hz}, J=9.2 \mathrm{~Hz}, 1 \mathrm{H}), 2.07-2.02(\mathrm{~m}, 1 \mathrm{H}), 1.08(\mathrm{t}, J$ $=7.2 \mathrm{~Hz}, 3 \mathrm{H})$.

${ }^{13} \mathrm{C}$ NMR $\left(100 \mathrm{MHz}, \mathrm{CDCl}_{3}\right) \delta 167.0,163.9,134.4,134.2,131.5,130.6,130.5,129.6,128.9,128.5,118.7,116.1,63.2,62.7,35.6,34.5,22.8,22.7$, $20.9,20.0,14.0,13.9$.

IR (ATR) 2985, 2926, 2245, 1731, $1264 \mathrm{~cm}^{-1}$.

HRMS (DART) Found 250.0641, Calcd. for $\mathrm{C}_{13} \mathrm{H}_{13} \mathrm{ClNO}_{2},[\mathrm{M}+\mathrm{H}]^{+} 250.0635$.

\section{Ethyl 1-cyano-2-(4-bromophenyl)cyclopropanecarboxylate (3h) (Table 2)}

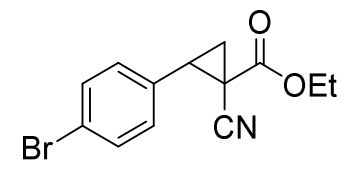

The product was isolated as a brown viscous oil (73.2 $\mathrm{mg}, 83 \%$ yield). Diastereomeric ratio (5:1) was determined by ${ }^{1} \mathrm{H}$ NMR analysis of the crude mixture.

$\mathrm{R}_{f}=0.43(20 \%$ EtOAc in $n$-Hexane)

${ }^{1} \mathrm{H}$ NMR (500 MHz, $\left.\mathrm{CDCl}_{3}\right) \delta$ Major 7.52-7.49 (m, $\left.2 \mathrm{H}\right), 7.17-7.13(\mathrm{~m}, 2 \mathrm{H}), 4.29(\mathrm{q}, J=6.9 \mathrm{~Hz}, 2 \mathrm{H}), 3.11(\mathrm{t}, J=8.6 \mathrm{~Hz}, 1 \mathrm{H}), 2.16(\mathrm{dd}, J=5.2$ $\mathrm{Hz}, J=9.2 \mathrm{~Hz}, 1 \mathrm{H}), 2.05(\mathrm{dd}, J=5.2 \mathrm{~Hz}, J=8.0 \mathrm{~Hz}, 1 \mathrm{H}), 1.35(\mathrm{t}, J=6.9 \mathrm{~Hz}, 3 \mathrm{H})$.

Minor 7.49-7.43 (m, $2 \mathrm{H}), 7.17-7.13(\mathrm{~m}, 2 \mathrm{H}), 4.01(\mathrm{q}, J=7.5 \mathrm{~Hz}, 2 \mathrm{H}), 3.21(\mathrm{~m}, 1 \mathrm{H}), 2.32(\mathrm{dd}, J=5.8 \mathrm{~Hz}, J=9.2 \mathrm{~Hz}, 1 \mathrm{H}), 2.06-2.02(\mathrm{~m}, 1 \mathrm{H})$, $1.25(\mathrm{t}, J=7.5 \mathrm{~Hz}, 3 \mathrm{H})$.

${ }^{13} \mathrm{C}$ NMR $\left(125 \mathrm{MHz}, \mathrm{CDCl}_{3}\right): \delta 166.9,163.9,131.9,131.8,131.4,131.0,130.8,129.9,122.5,116.0,63.1,62.6,60.3,35.6,34.4,22.7,22.6,20.8$, $19.9,14.1,14.0,13.8$.

IR (ATR) 2983, 2244, 1730, $1264 \mathrm{~cm}^{-1}$.

HRMS (DART) Found 294.0141, Calcd. for $\mathrm{C}_{13} \mathrm{H}_{13} \mathrm{BrNO}_{2},[\mathrm{M}+\mathrm{H}]^{+} 294.0130$. 
Ethyl 1-cyano-2-(4-nitrophenyl)cyclopropanecarboxylate (3i) (Table 2)

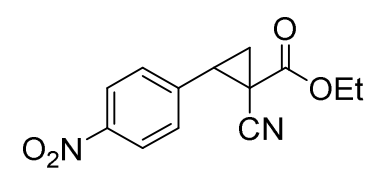

The product was isolated as a yellow oil $\left(70.3 \mathrm{mg}, 90 \%\right.$ yield). Diastereomeric ratio (7:1) was determined by ${ }^{1} \mathrm{H}$ NMR analysis of the crude mixture.

$\mathrm{R}_{f}=0.42(29 \%$ EtOAc in $n$-Hexane).

${ }^{1} \mathrm{H}$ NMR (400 MHz, $\mathrm{CDCl}_{3}$ ) $\delta$ Major 8.27-8.24 (m, $\left.2 \mathrm{H}\right), 7.50-7.46(\mathrm{~m}, 2 \mathrm{H}), 4.34(\mathrm{q}, J=7.2 \mathrm{~Hz}, 2 \mathrm{H}), 3.25(\mathrm{dd}, J=8.7 \mathrm{~Hz}, J=9.2 \mathrm{~Hz}, 1 \mathrm{H}), 2.26$ $(\mathrm{dd}, J=5.8 \mathrm{~Hz}, J=9.2 \mathrm{~Hz}, 1 \mathrm{H}), 2.15(\mathrm{dd}, J=5.8 \mathrm{~Hz}, J=8.7 \mathrm{~Hz}, 1 \mathrm{H}), 1.38(\mathrm{t}, J=7.2 \mathrm{~Hz}, 3 \mathrm{H})$.

Minor $8.19(\mathrm{~d}, J=8.7 \mathrm{~Hz}, 2 \mathrm{H}), 7.50-7.46(\mathrm{~m}, 2 \mathrm{H}), 4.04(\mathrm{q}, J=7.2 \mathrm{~Hz}, 2 \mathrm{H}), 3.35(\mathrm{t}, J=9.2 \mathrm{~Hz}, 1 \mathrm{H}), 2.41(\mathrm{dd}, J=5.8 \mathrm{~Hz}, J=9.2 \mathrm{~Hz}, 1 \mathrm{H})$, 2.18-2.14 (m, $1 \mathrm{H}), 1.11(\mathrm{t}, J=7.2 \mathrm{~Hz}, 3 \mathrm{H})$.

${ }^{13} \mathrm{C} \mathrm{NMR}\left(100 \mathrm{MHz}, \mathrm{CDCl}_{3}\right) \delta 166.5,163.7,147.8,147.6,140.3,139.4,130.3,129.3,123.9,123.4,118.1,115.6,63.4,63.0,35.2,33.9,23.2,22.9$, $21.1,20.3,14.0,13.9$.

IR (ATR) 3119, 2988, 2244, 1727, 1519, 1344, $1262 \mathrm{~cm}^{-1}$.

HRMS (DART) Found 261.0882, Calcd. for $\mathrm{C}_{13} \mathrm{H}_{13} \mathrm{~N}_{2} \mathrm{O}_{4},[\mathrm{M}+\mathrm{H}]^{+} 261.0875$.

\section{Ethyl 1-cyano-2-(2-pyridyl)cyclopropanecarboxylate (3j) (Table 2)}

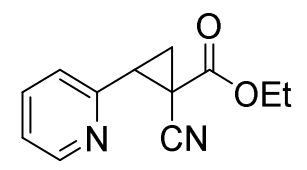

The product was isolated as a brown viscous oil $\left(17.5 \mathrm{mg}, 27 \%\right.$ yield). Diastereomeric ratio (5:1) was determined by ${ }^{1} \mathrm{H}$ NMR analysis of the crude mixture.

$\mathrm{R}_{f}=0.43(20 \%$ EtOAc in $n$-Hexane).

${ }^{1} \mathrm{H}$ NMR $\left(400 \mathrm{MHz}, \mathrm{CDCl}_{3}\right) \delta$ Major $8.60(\mathrm{~d}, J=3.9,1 \mathrm{H}), 7.71-7.67(\mathrm{~m}, 1 \mathrm{H}), 7.37(\mathrm{~d}, J=7.7,1 \mathrm{H}), 7.26-7.22(\mathrm{~m}, 1 \mathrm{H}), 4.30(\mathrm{q}, J=7.2 \mathrm{~Hz}, 2$ H), $3.23(\mathrm{dd}, J=8.2 \mathrm{~Hz}, J=9.2 \mathrm{~Hz}, 1 \mathrm{H}), 2.61(\mathrm{dd}, J=4.8 \mathrm{~Hz}, J=8.2 \mathrm{~Hz}, 1 \mathrm{H}), 2.12(\mathrm{dd}, J=4.8 \mathrm{~Hz}, J=9.2 \mathrm{~Hz}, 1 \mathrm{H}), 1.35(\mathrm{t}, J=7.2 \mathrm{~Hz}, 3 \mathrm{H})$. Minor $8.54(\mathrm{~m}, 1 \mathrm{H}), 7.71-7.67(\mathrm{~m}, 1 \mathrm{H}), 7.26-7.22(\mathrm{~m}, 2 \mathrm{H}), 4.01(\mathrm{q}, J=7.2 \mathrm{~Hz}, 2 \mathrm{H}), 3.40(\mathrm{~m}, 1 \mathrm{H}), 2.48(\mathrm{dd}, J=5.8 \mathrm{~Hz}, J=8.7 \mathrm{~Hz}, 1 \mathrm{H})$, 2.14-2.09 (m, $1 \mathrm{H}), 1.08(\mathrm{t}, J=7.2 \mathrm{~Hz}, 3 \mathrm{H})$.

${ }^{13} \mathrm{C} \mathrm{NMR}\left(100 \mathrm{MHz}, \mathrm{CDCl}_{3}\right) \delta 187.1,167.0,152.2,149.4,149.2,136.4,136.3,124.3,124.1,123.0,122.9,115.8,110.5,63.0,62.5,38.7,36.9$, $35.5,22.9,22.0,20.3,13.9,13.7,7.8$.

IR (ATR) 2986, 2245, 1729, $1262 \mathrm{~cm}^{-1}$.

HRMS (DART) Found 217.0997, Calcd. for $\mathrm{C}_{12} \mathrm{H}_{13} \mathrm{~N}_{2} \mathrm{O}_{2},[\mathrm{M}+\mathrm{H}]^{+}$217.0977. 


\section{Ethyl 1-cyano-2-methyl-2-phenylcyclopropanecarboxylate (3k) (Table 2)}

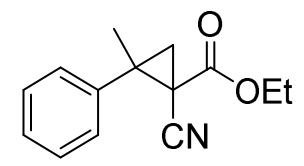

The product was isolated as a clear oil $\left(47.5 \mathrm{mg}, 69 \%\right.$ yield). Diastereomeric ratio $(1.3: 1)$ was determined by ${ }^{1} \mathrm{H}$ NMR analysis of the crude mixture.

$\mathrm{R}_{f}=0.38(20 \%$ EtOAc in $n$-Hexane $)$.

${ }^{1} \mathrm{H}$ NMR $\left(500 \mathrm{MHz}, \mathrm{CDCl}_{3}\right) \delta$ Major 7.41-7.23 (m, $\left.5 \mathrm{H}\right), 3.92(\mathrm{~m}, 2 \mathrm{H}), 2.45(\mathrm{~d}, J=5.8 \mathrm{~Hz}, 1 \mathrm{H}), 1.76(\mathrm{~s}, 3 \mathrm{H}), 1.74(\mathrm{~d}, J=5.2 \mathrm{~Hz}, 1 \mathrm{H}), 1.01(\mathrm{t}, J$ $=6.9 \mathrm{~Hz}, 3 \mathrm{H})$.

Minor 7.41-7.23 (m, $5 \mathrm{H}), 4.32$ (q, $J=6.9 \mathrm{~Hz}, 2 \mathrm{H}), 2.13$ (d, $J=5.8 \mathrm{~Hz}, 1 \mathrm{H}), 2.10$ (d, J=5.8 Hz, $1 \mathrm{H}), 1.59(\mathrm{~s}, 3 \mathrm{H}), 1.37$ (t, $J=6.9 \mathrm{~Hz}, 3 \mathrm{H})$.

${ }^{13} \mathrm{C}$ NMR $\left(125 \mathrm{MHz}, \mathrm{CDCl}_{3}\right) \delta 166.1,164.9,140.1,138.7,129.1,128.9,128.7,128.4,128.3,128.0,118.1,117.8,63.0,62.5,41.4,40.8,27.4,27.4$, 26.7, 26.3, 26.2, 20.9, 14.3, 13.9 .

IR (ATR) 2982, 2934, 2240, 1733, $1260 \mathrm{~cm}^{-1}$.

HRMS (DART) Found 230.1179, Calcd. for $\mathrm{C}_{14} \mathrm{H}_{16} \mathrm{NO}_{2},[\mathrm{M}+\mathrm{H}]^{+} 230.1181$.

\section{Ethyl 1-cyano-2-methyl-3-phenylcyclopropanecarboxylate (3I) (Table 2)}

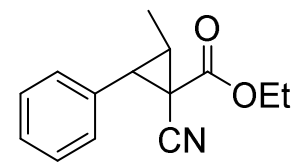

The product was isolated as a clear oil (44.7 mg, 65\% yield). Diastereomeric ratio (3.8:3.2:1) was determined by ${ }^{1} \mathrm{H}$ NMR analysis of the crude mixture.

$\mathrm{R}_{f}=0.52(20 \%$ EtOAc in $n$-Hexane)

${ }^{1} \mathrm{H}$ NMR $\left(500 \mathrm{MHz}, \mathrm{CDCl}_{3}\right) \delta$ Diastereoisomer $17.39-7.32(\mathrm{~m}, 5 \mathrm{H}), 4.31$ (q, $\left.J=6.9 \mathrm{~Hz}, 2 \mathrm{H}\right), 3.25(\mathrm{~d}, J=9.8 \mathrm{~Hz}, 1 \mathrm{H}), 2.34(\mathrm{dq}, J=6.3 \mathrm{~Hz}, J=$ $9.8 \mathrm{~Hz}, 1 \mathrm{H}), 1.38$ (t, $J=6.9 \mathrm{~Hz}, 3 \mathrm{H}), 1.30(\mathrm{~d}, J=6.3 \mathrm{~Hz}, 3 \mathrm{H})$.

Diastereoisomer 2 7.31-7.24 (m, 5 H), 3.99 (q, $J=7.2 \mathrm{~Hz}, 2 \mathrm{H}), 2.97$ (d, $J=8.2 \mathrm{~Hz}, 1 \mathrm{H}), 2.64(\mathrm{~m}, 1 \mathrm{H}), 1.54(\mathrm{~d}, J=6.3 \mathrm{~Hz}, 3 \mathrm{H}), 1.04(\mathrm{t}, J=7.2$ $\mathrm{Hz}, 3 \mathrm{H})$.

Diastereoisomer 3 7.39-7.24 (m, 5 H), 4.33-4.29 (m, 2 H), 3.14 (d, J=8.6 Hz, $1 \mathrm{H}), 2.46-2.45$ (m, $1 \mathrm{H}), 1.45(\mathrm{~d}, J=6.3 \mathrm{~Hz}, 3 \mathrm{H}), 1.28-1.24(\mathrm{~m}, 3$ H)

${ }^{13} \mathrm{C}$ NMR $\left(125 \mathrm{MHz}, \mathrm{CDCl}_{3}\right) \delta 168.2,164.5,132.5,131.4,130.0,129.2,128.9,128.4,128.3,128.1,117.6,115.8,63.1,62.5,44.2,38.5,30.4,27.9$, $26.7,26.2,15.1,14.2,14.0,11.5$ 
IR (ATR) 2982, 2937, 2241, 1731, $1255 \mathrm{~cm}^{-1}$.

HRMS (DART) Found 230.1171, Calcd. for $\mathrm{C}_{14} \mathrm{H}_{16} \mathrm{NO}_{2}$, [M+ H] $]^{+} 230.1181$.

\section{Ethyl 1-cyano-2,3-diphenylcyclopropanecarboxylate (3m) (Table 2)}

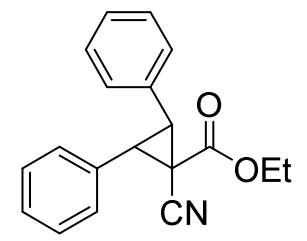

The product was isolated as a yellow oil $\left(35.0 \mathrm{mg}, 40 \%\right.$ yield). Diastereomeric ratio $(2.4: 1)$ was determined by ${ }^{1} \mathrm{H}$ NMR analysis of the crude mixture.

$\mathrm{R}_{f}=0.28(10 \%$ EtOAc in $n$-Hexane).

${ }^{1} \mathrm{H}$ NMR (500 MHz, $\left.\mathrm{CDCl}_{3}\right) \delta$ Major 7.28-7.25 (m, $\left.6 \mathrm{H}\right), 7.10-7.08(\mathrm{~m}, 4 \mathrm{H}), 4.37(\mathrm{q}, J=7.5 \mathrm{~Hz}, 2 \mathrm{H}), 3.51(\mathrm{~s}, 1 \mathrm{H}), 1.40(\mathrm{t}, J=7.5 \mathrm{~Hz}, 3 \mathrm{H})$.

Minor 7.42-7.35 (m, $10 \mathrm{H}), 3.96(\mathrm{dq}, J=4.0 \mathrm{~Hz}, J=7.5 \mathrm{~Hz}, 2 \mathrm{H}), 3.87(\mathrm{~d}, J=8.6 \mathrm{~Hz}, 1 \mathrm{H}), 3.71(\mathrm{~d}, J=9.2 \mathrm{~Hz}, 1 \mathrm{H}), 1.09$ (t, $J=7.5 \mathrm{~Hz}, 3 \mathrm{H})$.

${ }^{13} \mathrm{C}$ NMR $\left(125 \mathrm{MHz}, \mathrm{CDCl}_{3}\right) \delta 167.8,164.0,133.2,131.2,130.8,130.2,129.4,129.3,129.0,128.7,128.6,128.5,128.4,128.1,116.8,115.1,63.5$, $62.9,40.7,39.1,36.4,29.9,28.4,14.2,14.0$.

IR (ATR) 2985, 2243, 1730, $1246 \mathrm{~cm}^{-1}$.

HRMS (DART) Found 292.1351, Calcd. for $\mathrm{C}_{19} \mathrm{H}_{18} \mathrm{NO}_{2},[\mathrm{M}+\mathrm{H}]^{+} 292.1338$.

\section{Methyl 1-cyano-2-phenylcyclopropanecarboxylate (3p) (Table 3)}

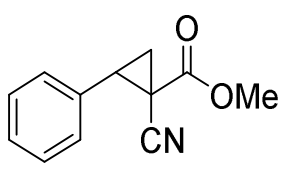

The product was isolated as a colorless oil ( $48.3 \mathrm{mg}, 80 \%$ yield). Diastereomeric ratio $(5.5: 1)$ was determined by ${ }^{1} \mathrm{H}$ NMR analysis of the crude mixture.

$\mathrm{R}_{f}=0.40$ (20\% EtOAc in $n$-Hexane).

${ }^{1} \mathrm{H}$ NMR (400 MHz, $\left.\mathrm{CDCl}_{3}\right) \delta$ Major 7.41-7.26 (m, $\left.5 \mathrm{H}\right), 3.87$ (s, $\left.3 \mathrm{H}\right), 3.19(\mathrm{dd}, J=7.7 \mathrm{~Hz}, J=8.2 \mathrm{~Hz}, 1 \mathrm{H}), 2.19-2.11(\mathrm{~m}, 2 \mathrm{H})$.

Minor 7.41-7.26 (m, 5 H), $3.55(\mathrm{~s}, 3 \mathrm{H}), 3.31(\mathrm{~m}, 1 \mathrm{H}), 2.40-2.36(\mathrm{~m}, 1 \mathrm{H}), 2.07-2.04(\mathrm{~m}, 1 \mathrm{H})$.

${ }^{13} \mathrm{C} \mathrm{NMR}\left(100 \mathrm{MHz}, \mathrm{CDCl}_{3}\right) \delta 167.8,132.7,129.2,128.9,128.7,128.6,128.5,128.4,128.3,128.2,128.1,125.9,116.3,53.7,53.2,36.6,35.5$, $22.8,22.7,20.2$.

IR (ATR) 3032, 2956, 2244, 1733, $1265 \mathrm{~cm}^{-1}$. 
HRMS (DART) Found 202.0874, Calcd. for $\mathrm{C}_{12} \mathrm{H}_{12} \mathrm{NO}_{2},[\mathrm{M}+\mathrm{H}]^{+} 202.0868$.

\section{1-Cyano-2-phenylcyclopropane-2-furyl ketone (3q) (Table 3)}

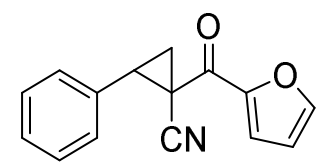

The product was isolated as a yellow solid (33.5 mg, 47\% yield). Diastereomeric ratio (12:1) was determined by ${ }^{1} \mathrm{H}$ NMR analysis of the crude mixture.

$\mathrm{R}_{f}=0.40(20 \%$ EtOAc in $n$-Hexane).

${ }^{1} \mathrm{H}$ NMR (400 MHz, $\left.\mathrm{CDCl}_{3}\right) \delta$ Major 7.80-7.71 (m, $\left.2 \mathrm{H}\right), 7.47-7.16(\mathrm{~m}, 5 \mathrm{H}), 6.61-6.60(\mathrm{~m}, 1 \mathrm{H}), 3.27(\mathrm{dd}, J=8.7 \mathrm{~Hz}, J=9.2 \mathrm{~Hz}, 1 \mathrm{H}), 2.48(\mathrm{dd}, J$ $=4.8 \mathrm{~Hz}, J=9.2 \mathrm{~Hz}, 1 \mathrm{H}), 2.21(\mathrm{dd}, J=4.8 \mathrm{~Hz}, J=8.7 \mathrm{~Hz}, 1 \mathrm{H})$.

Minor $7.61(\mathrm{~s}, 2 \mathrm{H}), 7.47-7.16(\mathrm{~m}, 5 \mathrm{H}), 6.52-6.51(\mathrm{~m}, 1 \mathrm{H}), 3.48(\mathrm{t}, J=9.2 \mathrm{~Hz}, 1 \mathrm{H}), 2.74(\mathrm{dd}, J=5.8 \mathrm{~Hz}, J=8.7 \mathrm{~Hz}, 1 \mathrm{H}), 2.01(\mathrm{dd}, J=5.8 \mathrm{~Hz}, J$ $=9.2 \mathrm{~Hz}, 1 \mathrm{H})$.

${ }^{13} \mathrm{C}$ NMR $\left(100 \mathrm{MHz}, \mathrm{CDCl}_{3}\right) \delta 177.8,174.9,150.2,147.9,147.5,133.0,128.9,128.7,128.6,128.4,128.3,128.2,128.1,125.7,121.2,120.5,120.1$, $118.4,112.7,112.5,82.0,38.6,38.3,27.2,23.8,18.0,16.6,5.3$.

IR (ATR) 3094, 3060, 2925, 2235, 1652, $1275 \mathrm{~cm}^{-1}$.

HRMS (DART) Found 238.0870, Calcd. for $\mathrm{C}_{15} \mathrm{H}_{12} \mathrm{NO}_{2},[\mathrm{M}+\mathrm{H}]^{+} 238.0868$.

Melting point $70-73{ }^{\circ} \mathrm{C}$.

\section{1-Cyano-2-phenylcyclopropane-2-toluoyl ketone (3r) (Table 3)}

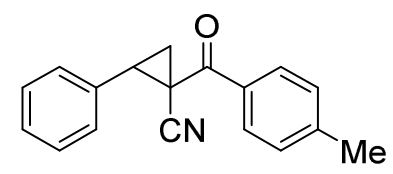

The product was isolated as a yellow viscous oil $\left(40.0 \mathrm{mg}, 51 \%\right.$ yield). Diastereomeric ratio (11:1) was determined by ${ }^{1} \mathrm{H}$ NMR analysis of the crude mixture.

$\mathrm{R}_{f}=0.41(20 \%$ EtOAc in $n$-Hexane).

${ }^{1} \mathrm{H}$ NMR $\left(500 \mathrm{MHz}, \mathrm{CDCl}_{3}\right) \delta$ Major $7.88(\mathrm{~d}, J=8.1 \mathrm{~Hz}, 2 \mathrm{H}), 7.45-7.19(\mathrm{~m}, 7 \mathrm{H}), 3.12(\mathrm{dd}, J=8.6 \mathrm{~Hz}, J=9.2 \mathrm{~Hz}, 1 \mathrm{H}), 2.52(\mathrm{dd}, J=5.2 \mathrm{~Hz}, J=$ $9.2 \mathrm{~Hz}, 1 \mathrm{H}), 2.41(\mathrm{~s}, 3 \mathrm{H}), 2.18(\mathrm{dd}, J=5.2 \mathrm{~Hz}, J=8.6 \mathrm{~Hz}, 1 \mathrm{H})$.

Minor $7.78(\mathrm{~d}, J=8.0 \mathrm{~Hz}, 2 \mathrm{H}), 7.45-7.19(\mathrm{~m}, 7 \mathrm{H}), 3.50(\mathrm{~m}, 1 \mathrm{H}), 2.77(\mathrm{dd}, J=5.8 \mathrm{~Hz}, J=8.6 \mathrm{~Hz}, 1 \mathrm{H}), 2.36(\mathrm{~s}, 3 \mathrm{H}), 2.02(\mathrm{dd}, J=5.7 \mathrm{~Hz}, J=$ $9.1 \mathrm{~Hz}, 1 \mathrm{H})$.

${ }^{13} \mathrm{C}$ NMR $\left(125 \mathrm{MHz}, \mathrm{CDCl}_{3}\right) \delta 190.9,171.1,151.7,144.7,133.2,132.8,129.3,129.2,129.0,128.9,128.8,128.7,128.5,128.3,128.2,128.1,119.3$, $118.7,38.5,37.5,29.6,28.4,23.8,22.0,21.7,21.0,17.8,14.1$.

IR (ATR) 3032, 2237, 1673, $1267 \mathrm{~cm}^{-1}$.

HRMS (DART) Found 262.1231, Calcd. for $\mathrm{C}_{18} \mathrm{H}_{16} \mathrm{NO}$, [M+ H] $]^{+} 262.1232$. 


\section{1-Cyano-2-phenylcyclopropane-2-pivaloyl ketone (3s) (Table 3)}

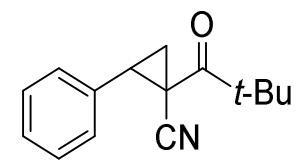

The product was isolated as a yellow viscous oil (50.5 mg, 74\% yield). Diastereomeric ratio (11.5:1) was determined by ${ }^{1} \mathrm{H}$ NMR analysis of the crude mixture.

$\mathrm{R}_{f}=0.67$ (20\% EtOAc in $n$-Hexane).

${ }^{1} \mathrm{H}$ NMR (400 MHz, $\left.\mathrm{CDCl}_{3}\right) \delta$ 7.40-7.24 (m, $\left.5 \mathrm{H}\right), 2.96(\mathrm{t}, J=8.7 \mathrm{~Hz}, 1 \mathrm{H}), 2.26(\mathrm{dd}, J=4.8 \mathrm{~Hz}, J=9.2 \mathrm{~Hz}, 1 \mathrm{H}), 2.05(\mathrm{dd}, J=4.8 \mathrm{~Hz}, J=8.2 \mathrm{~Hz}$, $1 \mathrm{H}), 1.39$ (s, $9 \mathrm{H})$.

${ }^{13} \mathrm{C} \mathrm{NMR}\left(100 \mathrm{MHz}, \mathrm{CDCl}_{3}\right) \delta 133.2,129.2,128.7,128.4,119.2,45.3,39.0,28.4,26.4,26.1,24.2$.

IR (ATR) 2975, 2239, 1693, 1267, $1189 \mathrm{~cm}^{-1}$.

HRMS (DART) Found 228.1398, Calcd. for $\mathrm{C}_{18} \mathrm{H}_{18} \mathrm{NO},[\mathrm{M}+\mathrm{H}]^{+} 228.1388$.

\section{1,1-Dicyano-2-phenylcyclopropane (3t) (Table 3) ${ }^{2}$}

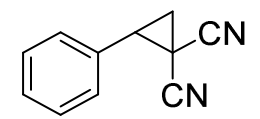

The product was isolated as a yellow oil ( $37.8 \mathrm{mg}, 75 \%$ yield).

$\mathrm{R}_{f}=0.34(20 \%$ EtOAc in $n$-Hexane).

${ }^{1} \mathrm{H}$ NMR (400 MHz, $\mathrm{CDCl}_{3}$ ) $\delta$ 7.43-7.26 (m, $\left.5 \mathrm{H}\right), 3.30$ (t, $\left.J=9.2 \mathrm{~Hz}, 1 \mathrm{H}\right), 2.29-2.22(\mathrm{~m}, 2 \mathrm{H})$.

${ }^{13} \mathrm{C}$ NMR $\left(100 \mathrm{MHz}, \mathrm{CDCl}_{3}\right) \delta 130.5,129.5,129.1,128.3,115.3,113.0,35.1,22.3,7.2$.

\section{2-Phenyl-1,1-cyclopropanedicarboxylic acid diethyl ester (3u) (Table 3) ${ }^{3}$}

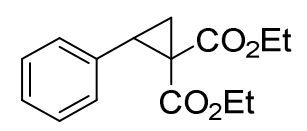

The product was isolated as a colorless oil ( $40.1 \mathrm{mg}, 51 \%$ yield).

$\mathrm{R}_{f}=0.39(20 \%$ EtOAc in $n$-Hexane).

${ }^{1} \mathrm{H}$ NMR $\left(400 \mathrm{MHz}, \mathrm{CDCl}_{3}\right) \delta$ 7.26-7.20 (m, $\left.5 \mathrm{H}\right), 4.29-4.17(\mathrm{~m}, 2 \mathrm{H}), 3.84(\mathrm{q}, J=7.2 \mathrm{~Hz}, 2 \mathrm{H}), 3.22(\mathrm{t}, J=8.7 \mathrm{~Hz}, 1 \mathrm{H}), 2.18(\mathrm{dd}, J=4.8 \mathrm{~Hz}, J=$ $8.2 \mathrm{~Hz}, 1 \mathrm{H}), 1.70(\mathrm{dd}, J=4.8 \mathrm{~Hz}, J=9.2 \mathrm{~Hz}, 1 \mathrm{H}), 1.29(\mathrm{t}, J=7.2 \mathrm{~Hz}, 3 \mathrm{H}), 0.85(\mathrm{t}, J=7.2 \mathrm{~Hz}, 3 \mathrm{H})$.

${ }^{13} \mathrm{C}$ NMR $\left(100 \mathrm{MHz}, \mathrm{CDCl}_{3}\right) \delta 169.8,166.6,134.6,128.5,128.1,127.3,61.7,61.1,37.4,32.1,18.7,14.0,13.6$. 


\section{Reference:}

${ }^{1}$ Marcoux, D.; Azzi, Charette, A. B. J. Am. Chem. Soc., 2009, 131, 6970.

${ }^{2}$ Gopinath, P.; chandrasekaran, S. J. Org. Chem. 2011, 76, 700.

${ }^{3}$ Goudreau, S. R.; Marcoux, D.; Charette, A. B. J. Org. Chem., 2009, 74, 470. 


\section{5. ${ }^{1} \mathrm{H}$ and ${ }^{13} \mathrm{C}$ spectra}

Ethyl 1-cyano-2-phenylcyclopropanecarboxylate (3a)<smiles>CCOC(=O)C1(C#N)CC1[PH2]c1ccccc1</smiles>
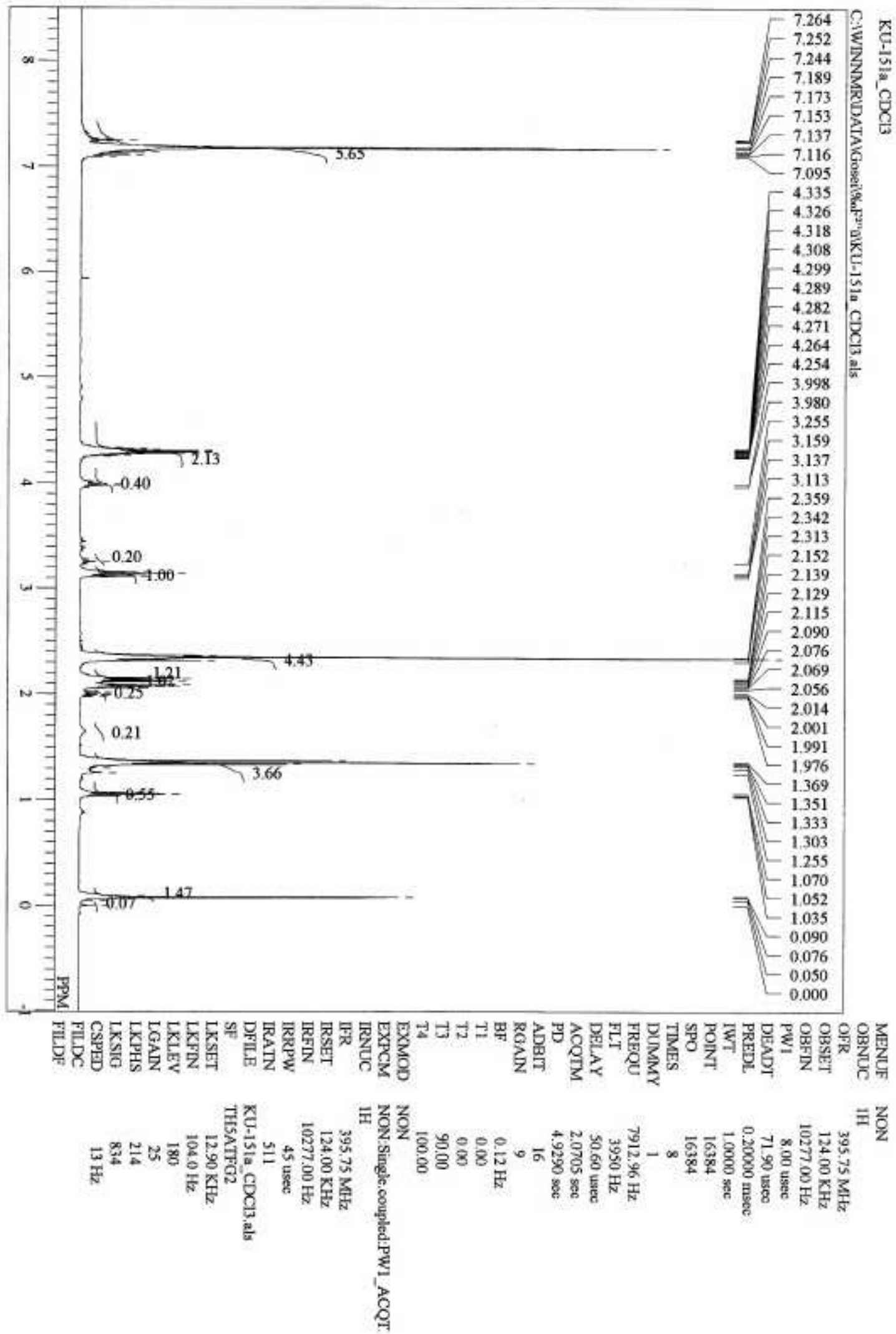


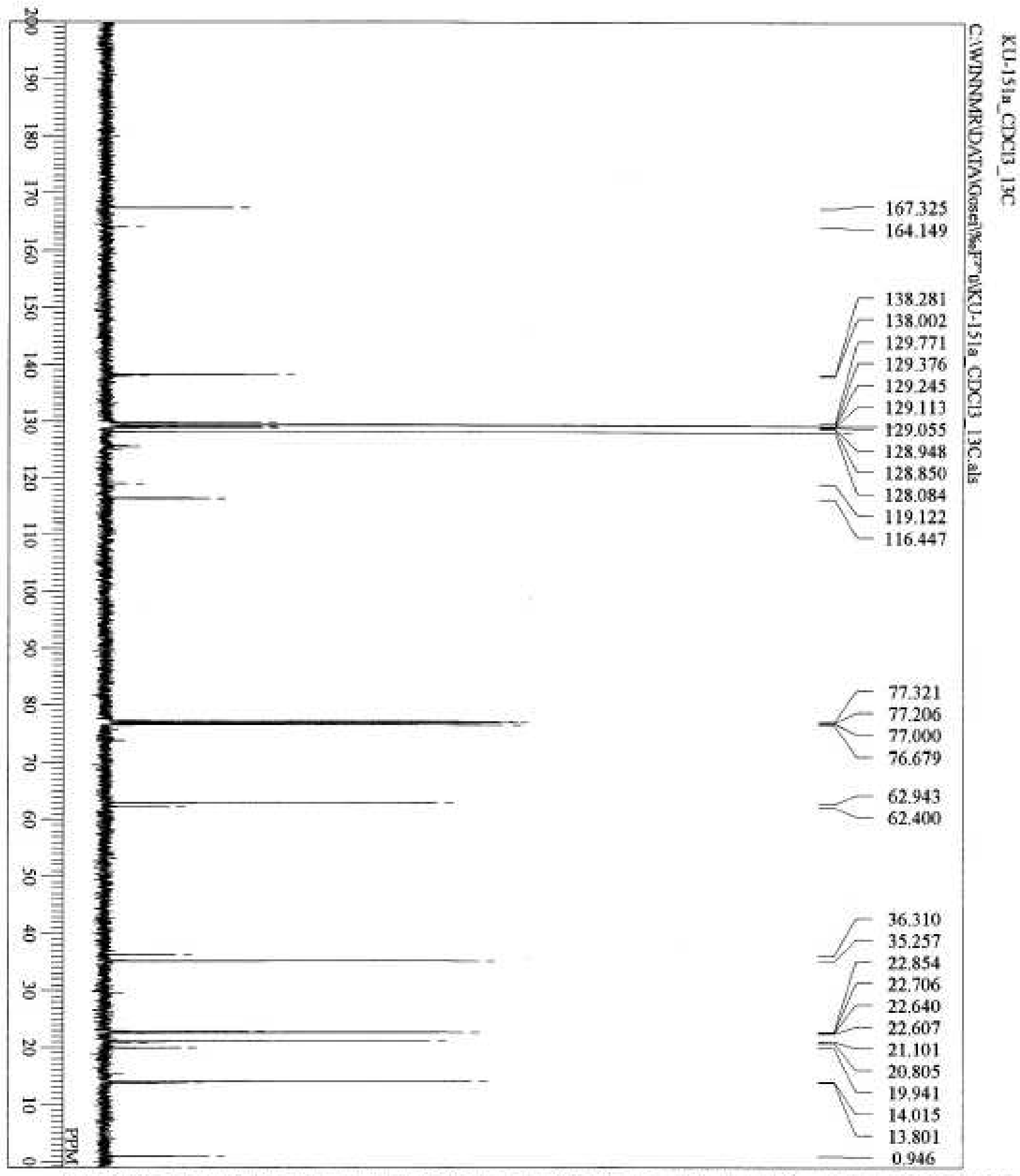

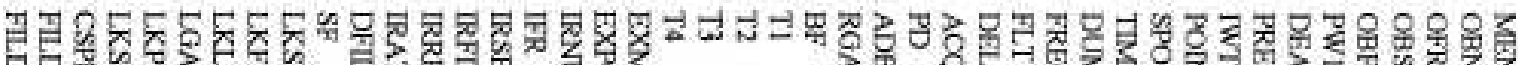

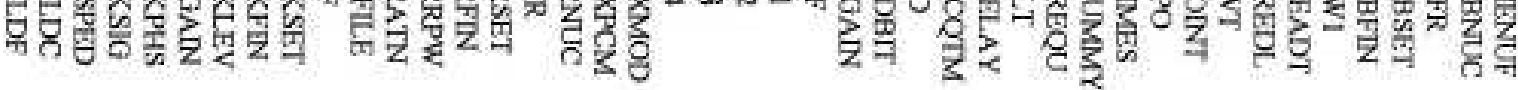

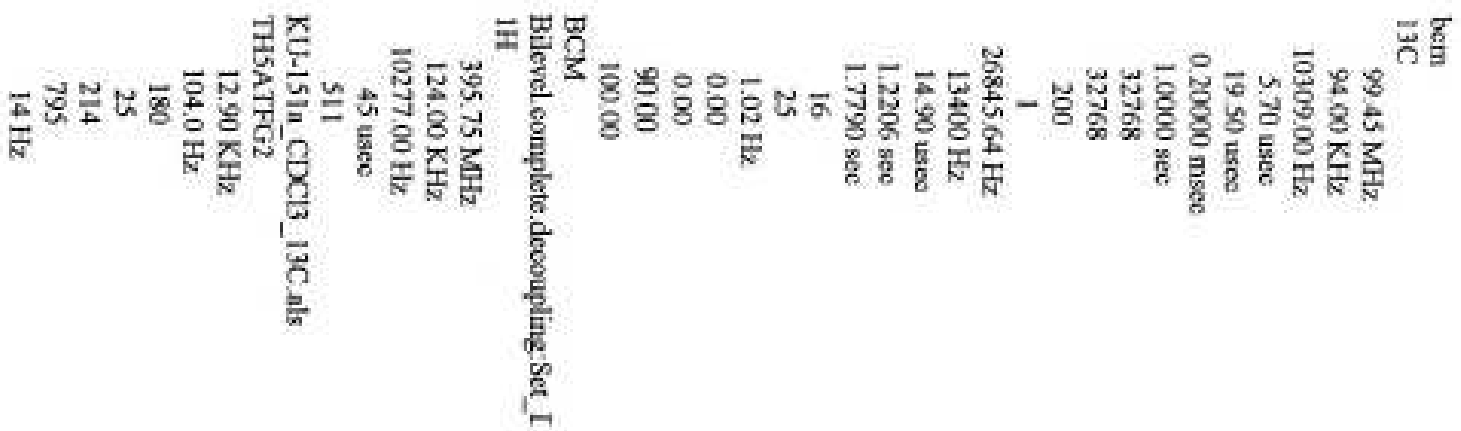


Ethyl 1-cyano-2-(4-methoxyphenyl)cyclopropanecarboxylate (3b)<smiles>CCOC(=O)C1(C#N)CC1c1ccc(OC)cc1</smiles>

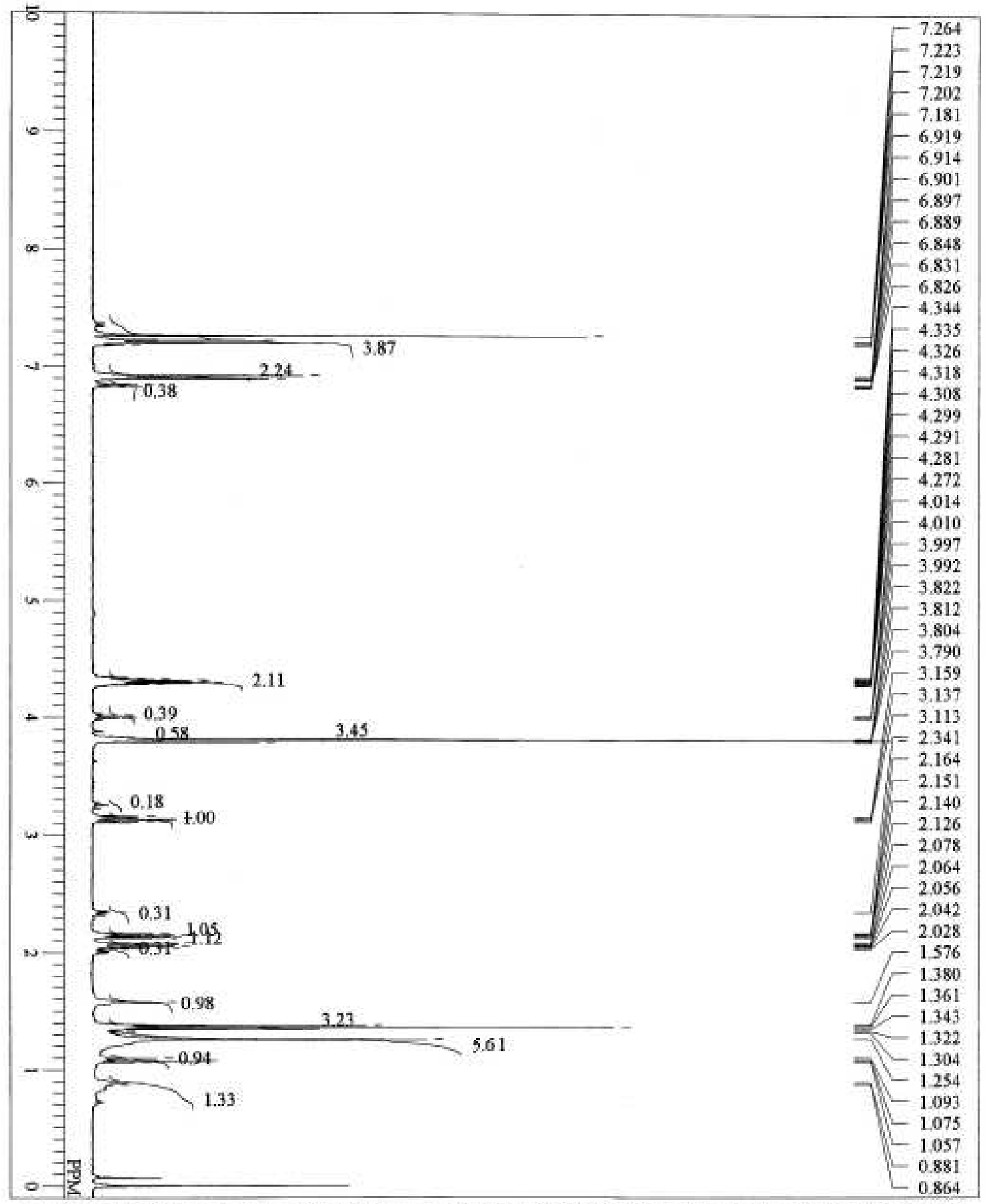

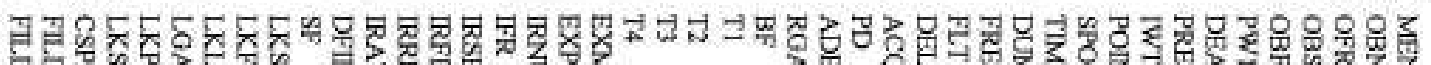

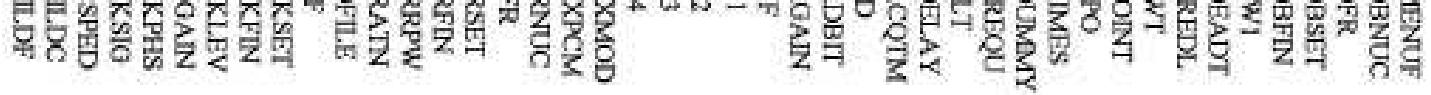

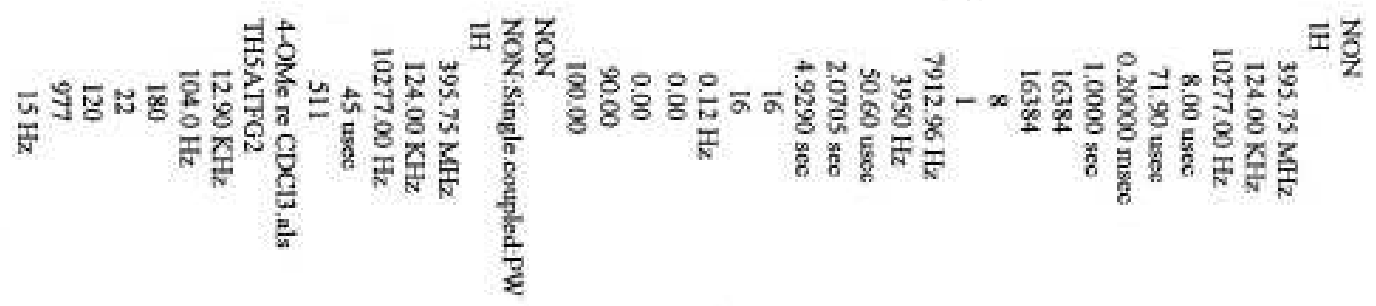




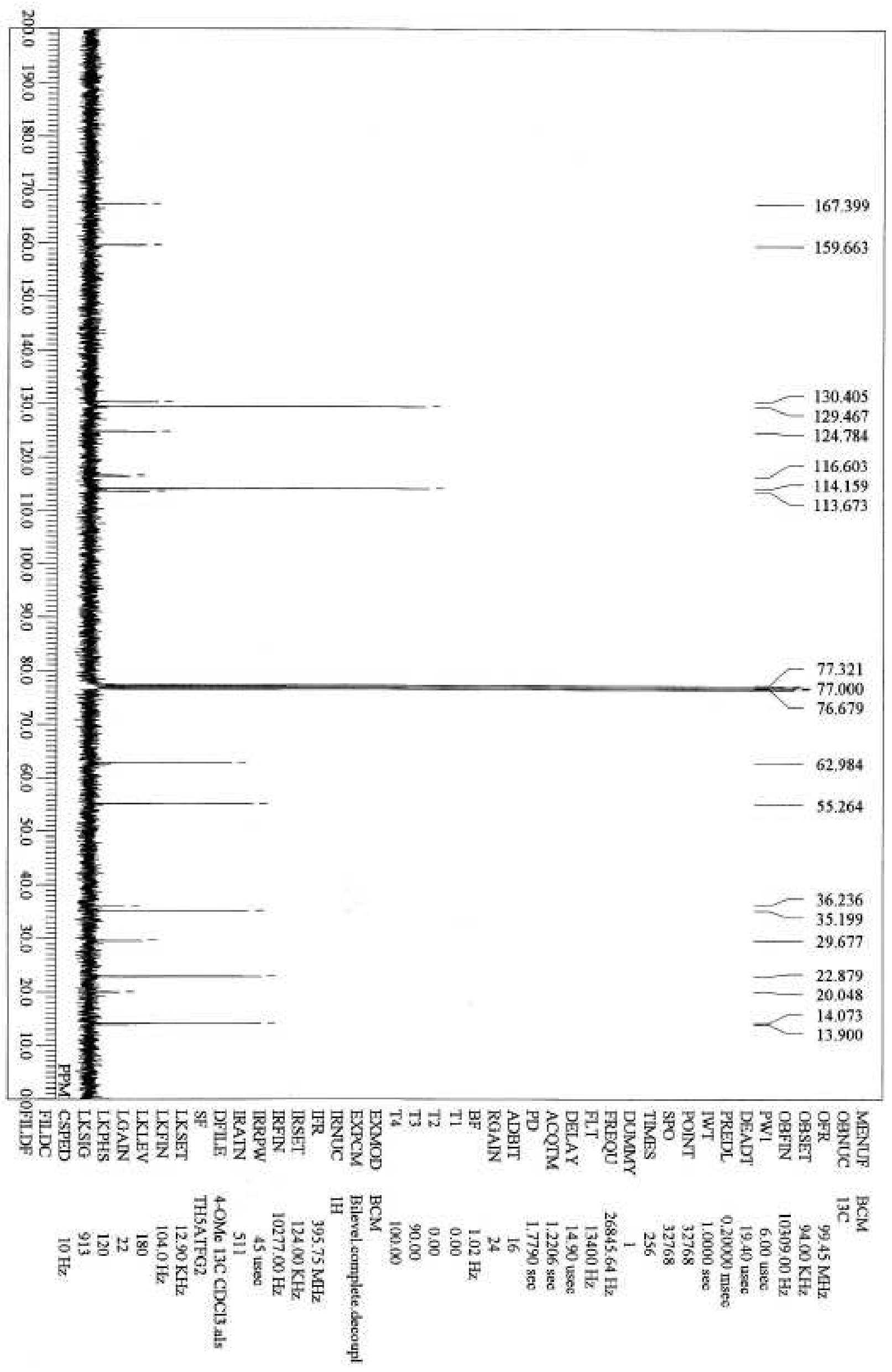


Ethyl 1-cyano-2-(4-tert-butylphenyl)cyclopropanecarboxylate (3c)<smiles>CCOC(=O)C1(C)CC1c1ccc(C(C)(C)C)cc1</smiles>

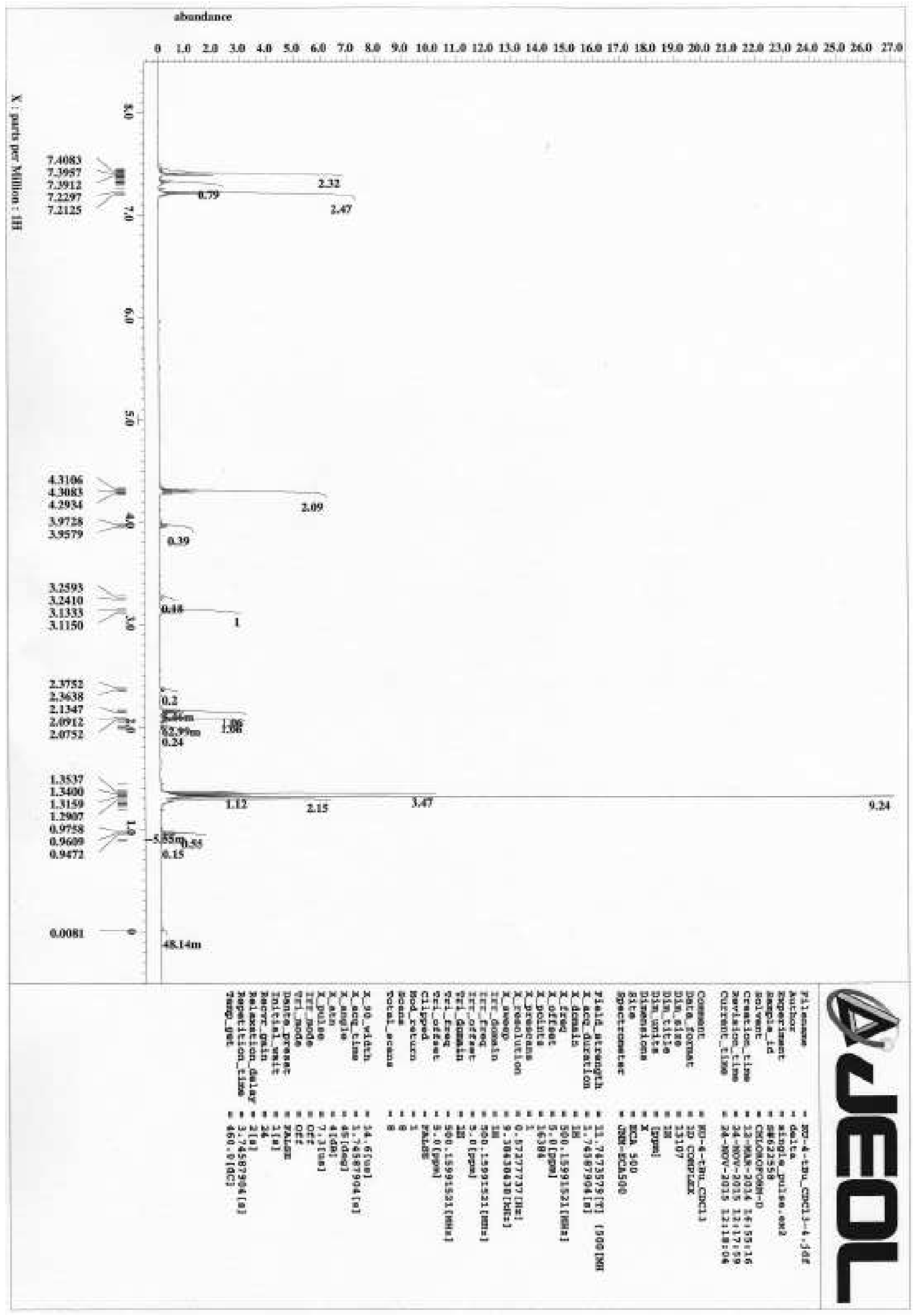




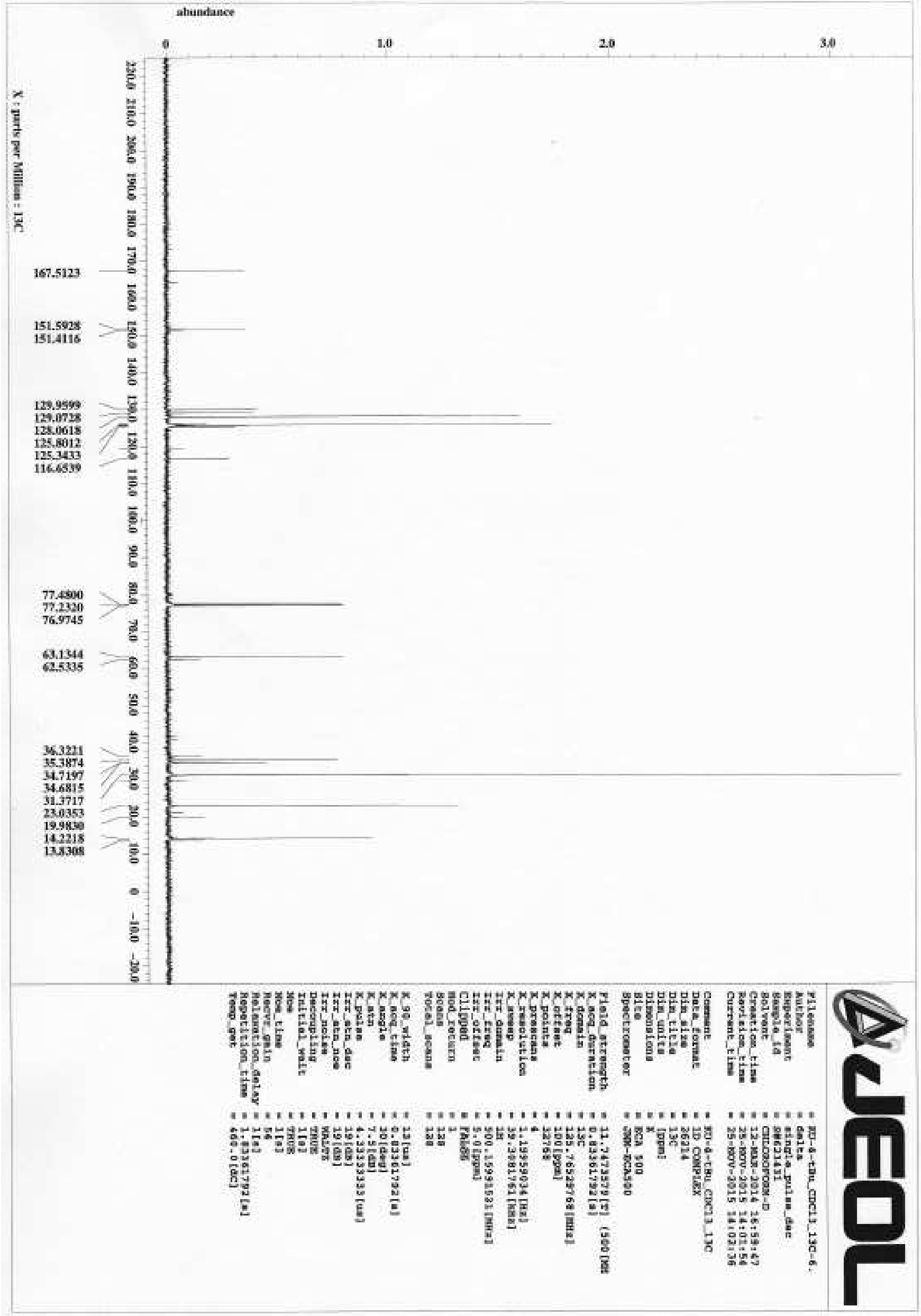


Ethyl 1-cyano-2-(4-methylphenyl)cyclopropanecarboxylate (3d)<smiles></smiles>

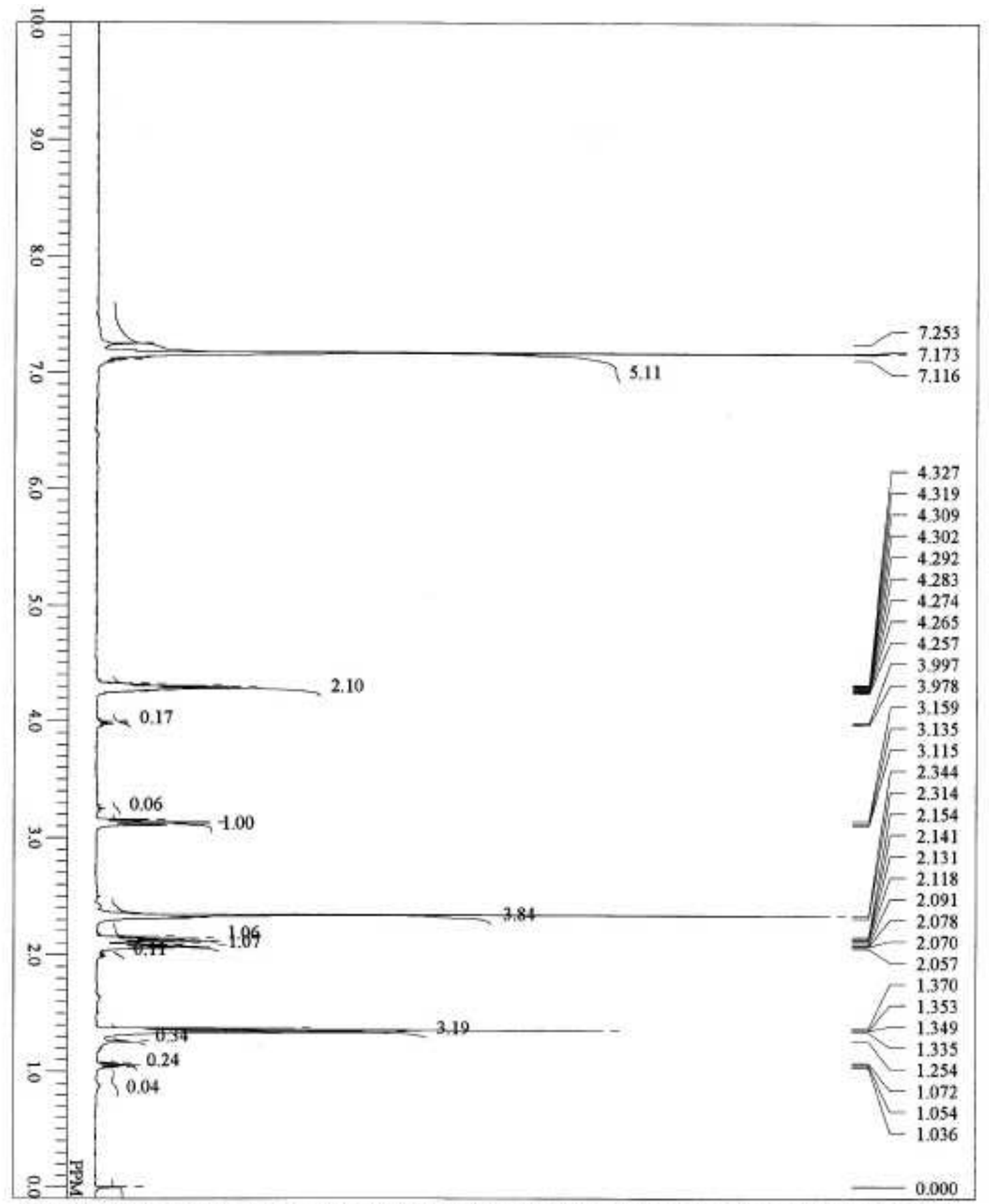

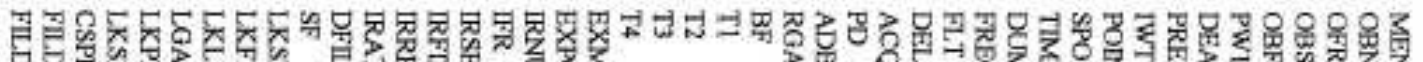

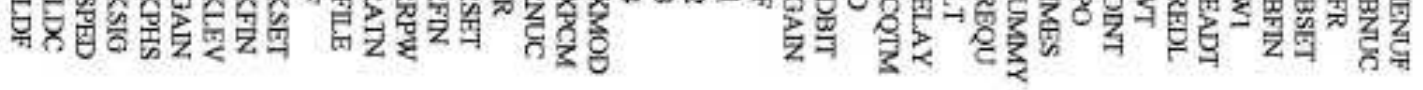

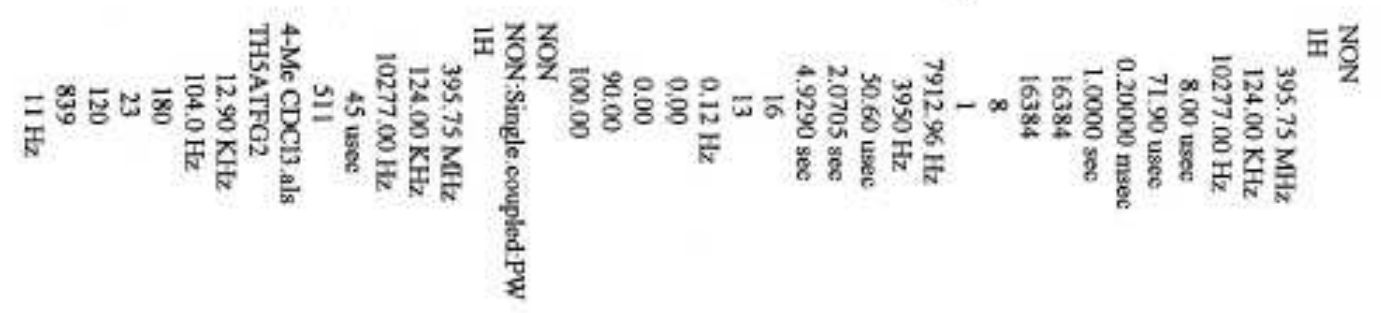




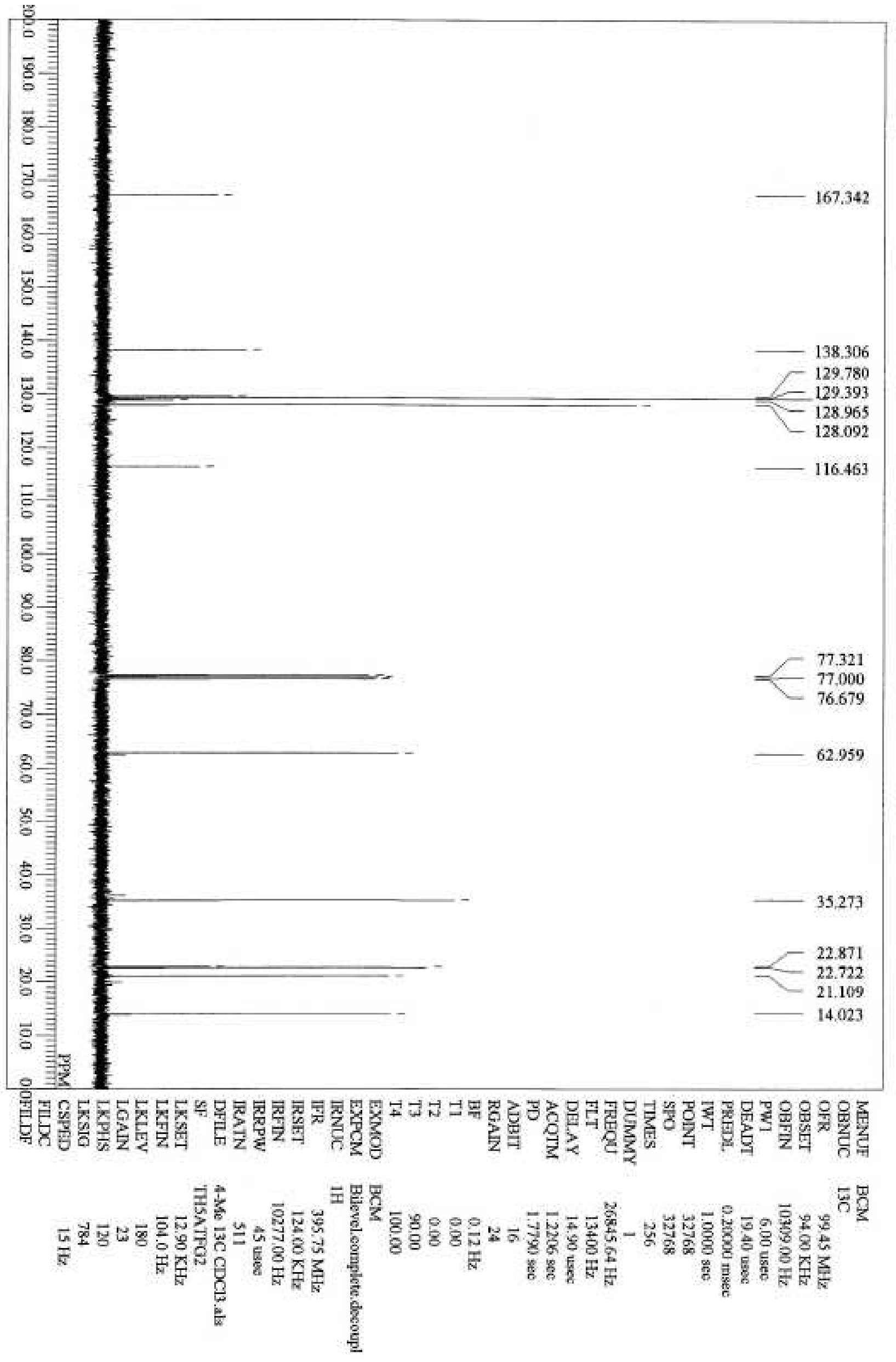


Ethyl 1-cyano-2-(2-methylphenyl)cyclopropanecarboxylate (3e)<smiles>CCOC(=O)C1(C#N)CC1c1ccccc1C</smiles>

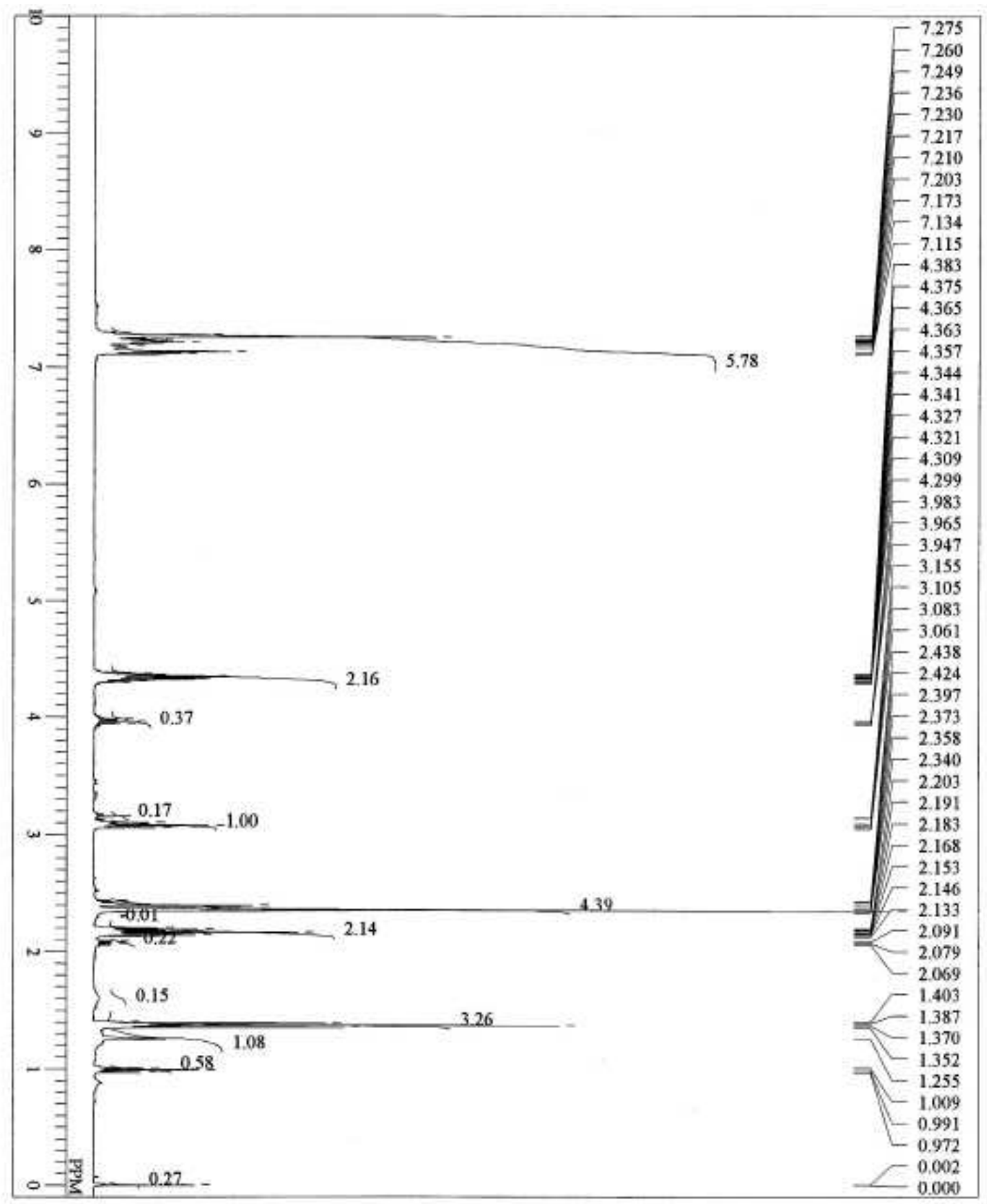

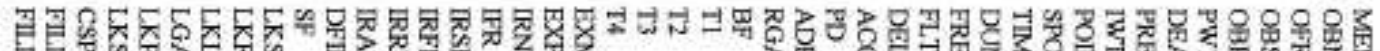

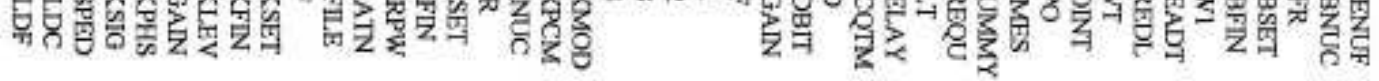

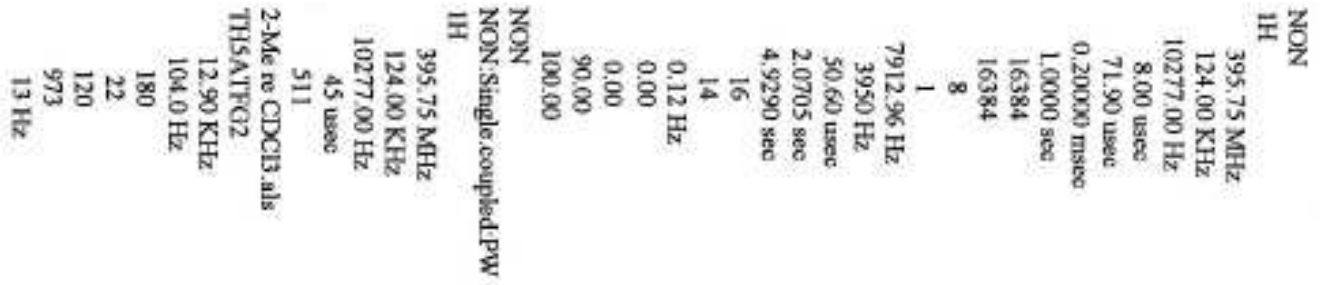




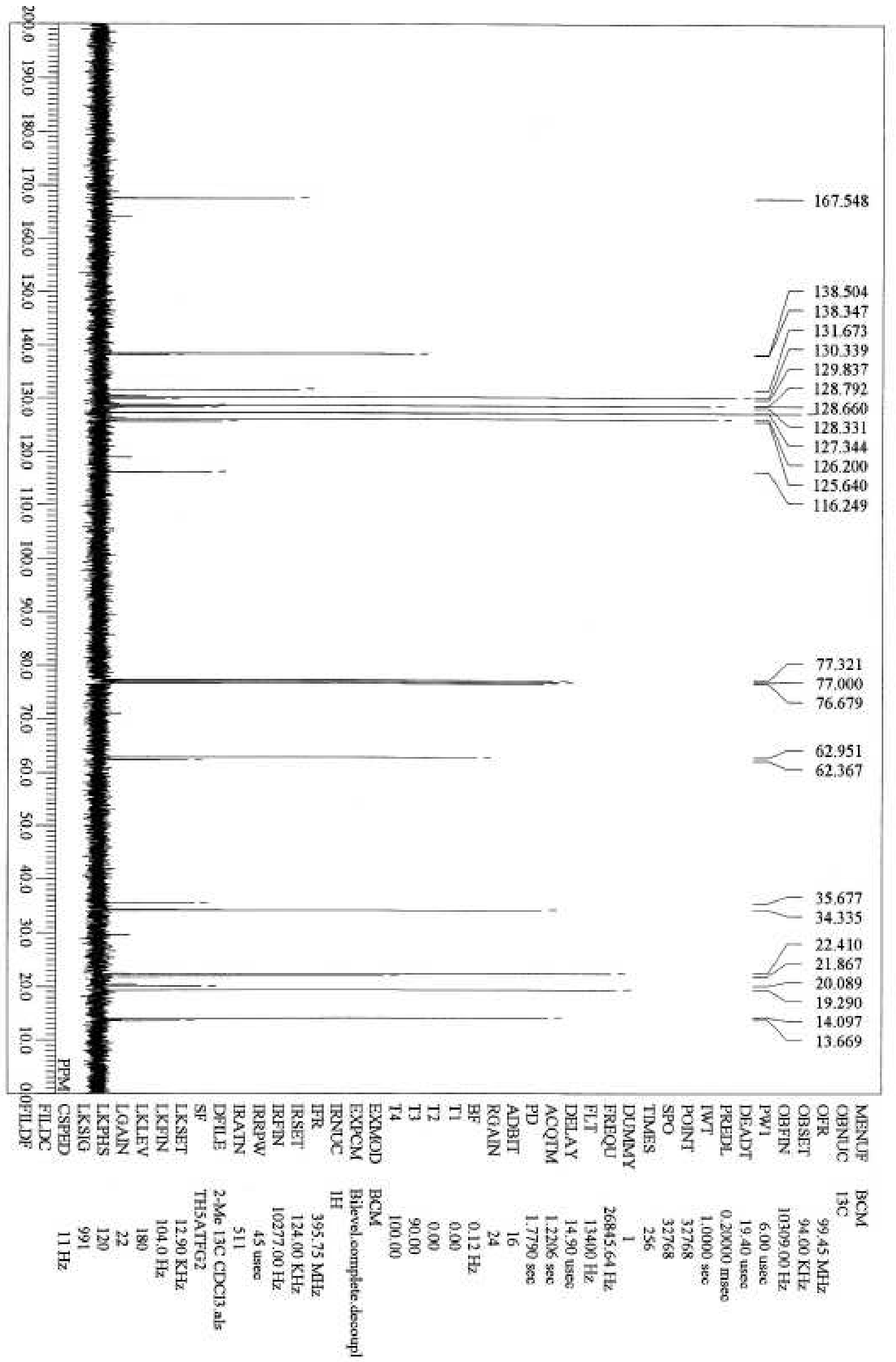


Ethyl 1-cyano-2-(3-methylphenyl)cyclopropanecarboxylate (3f)<smiles>CCOC(=O)C1(C#N)CC1c1cccc(C)c1</smiles>

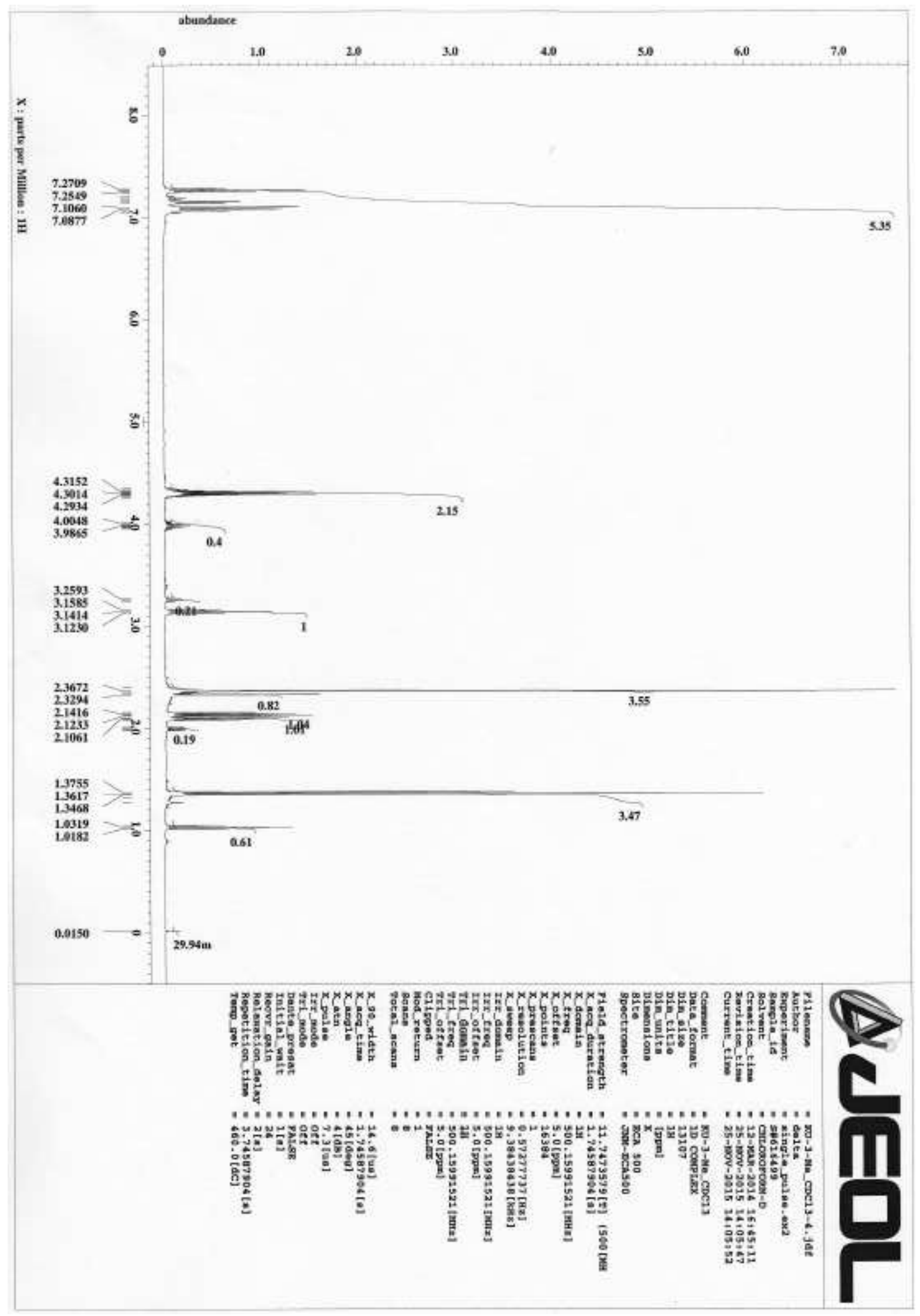




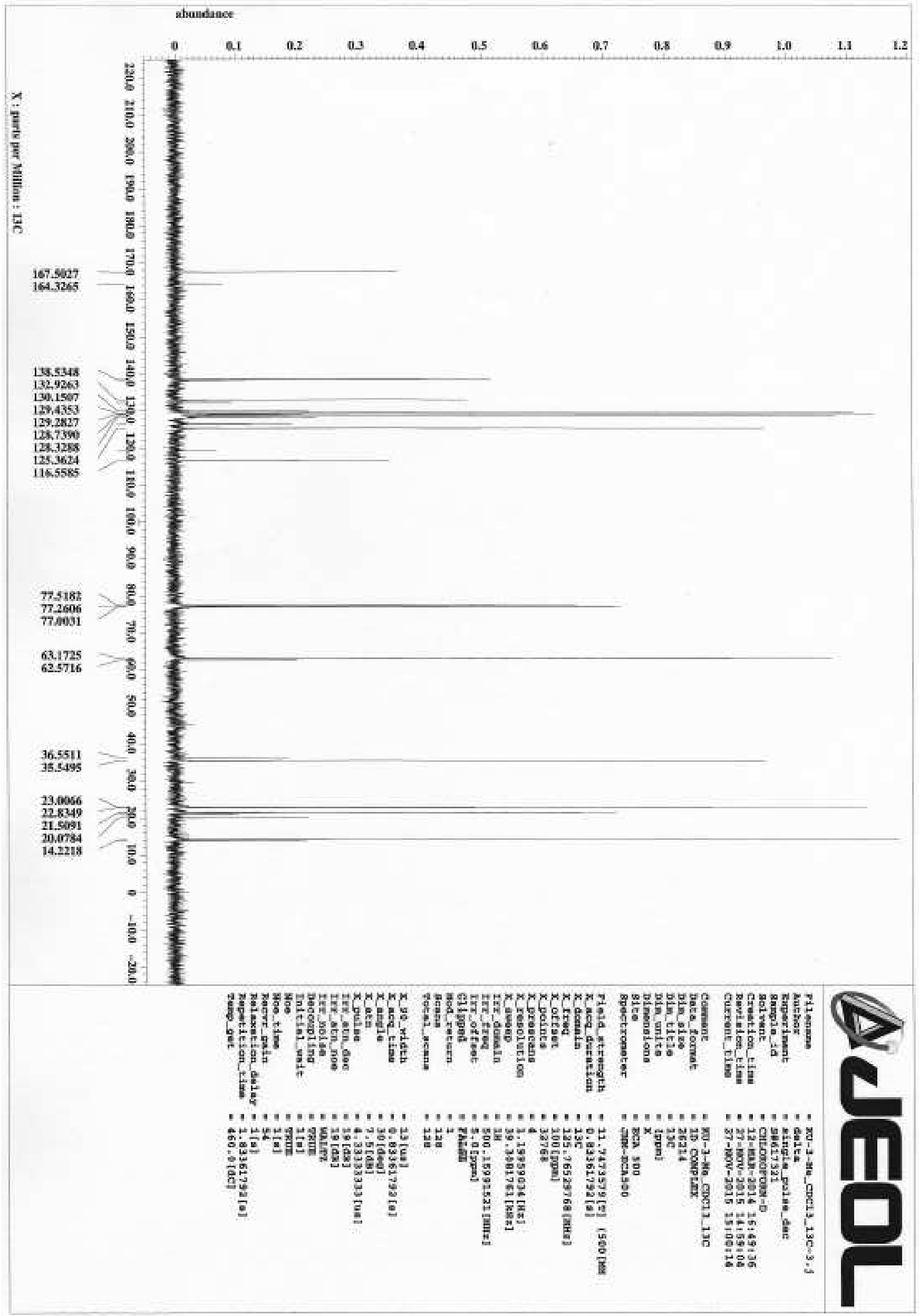




\section{Ethyl 1-cyano-2-(4-chrolophenyl)cyclopropanecarboxylate (3g)}<smiles>CCOC(=O)C1(C#N)CC1c1ccc(Cl)cc1</smiles>

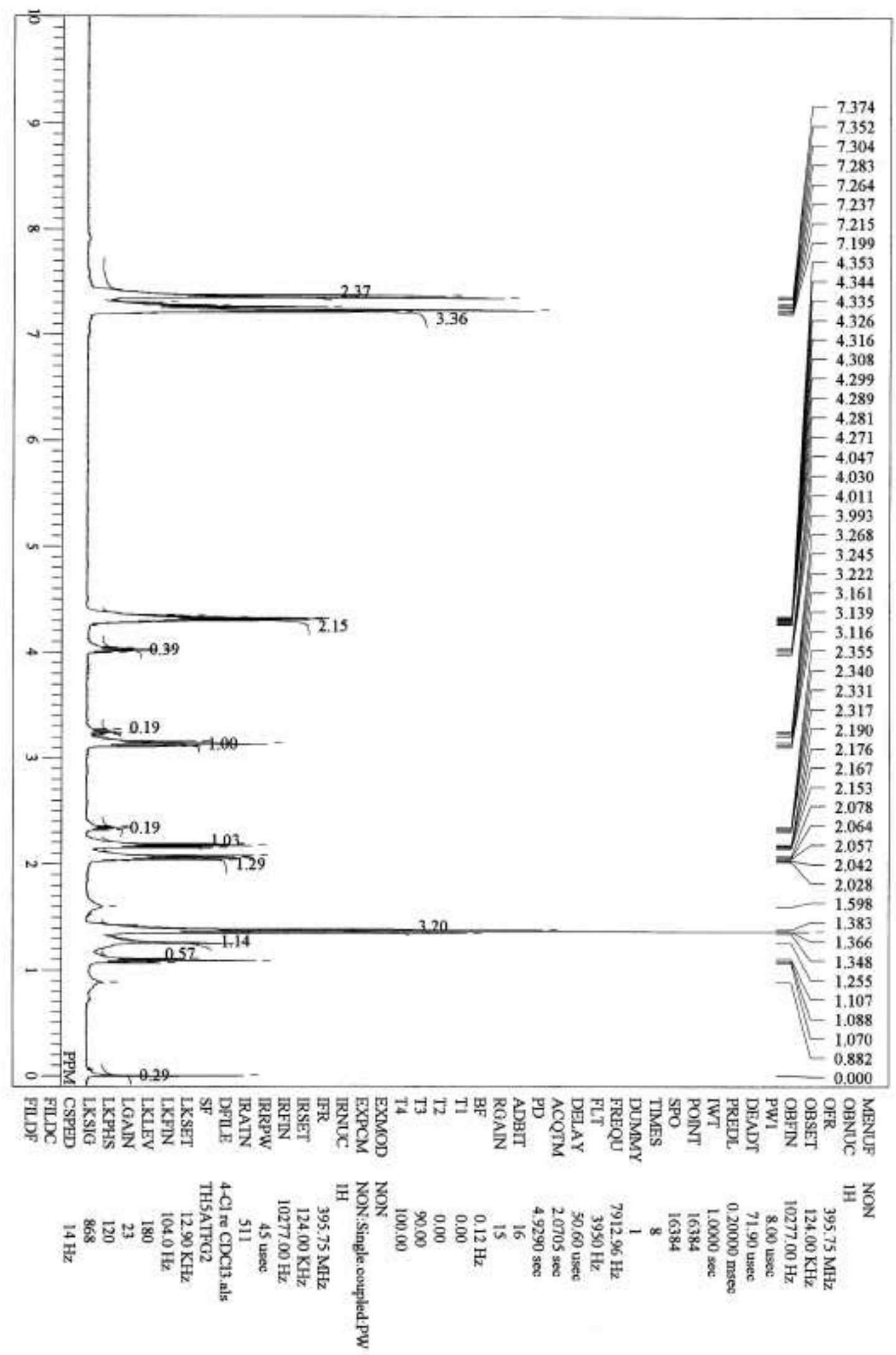







Ethyl 1-cyano-2-(4-bromophenyl)cyclopropanecarboxylate (3h)<smiles>CCOC(=O)C1(C)CC1c1ccc(Br)cc1</smiles>

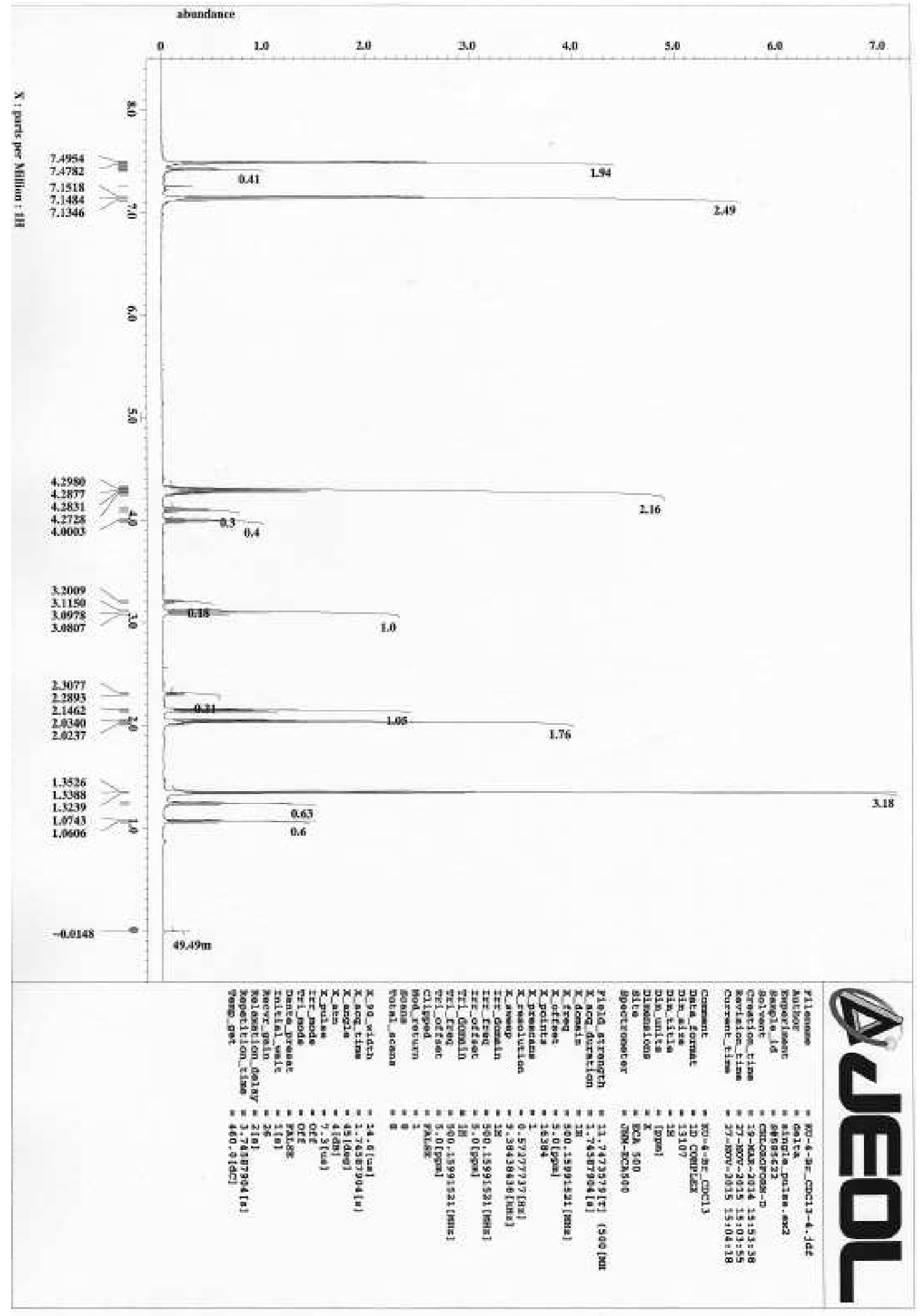




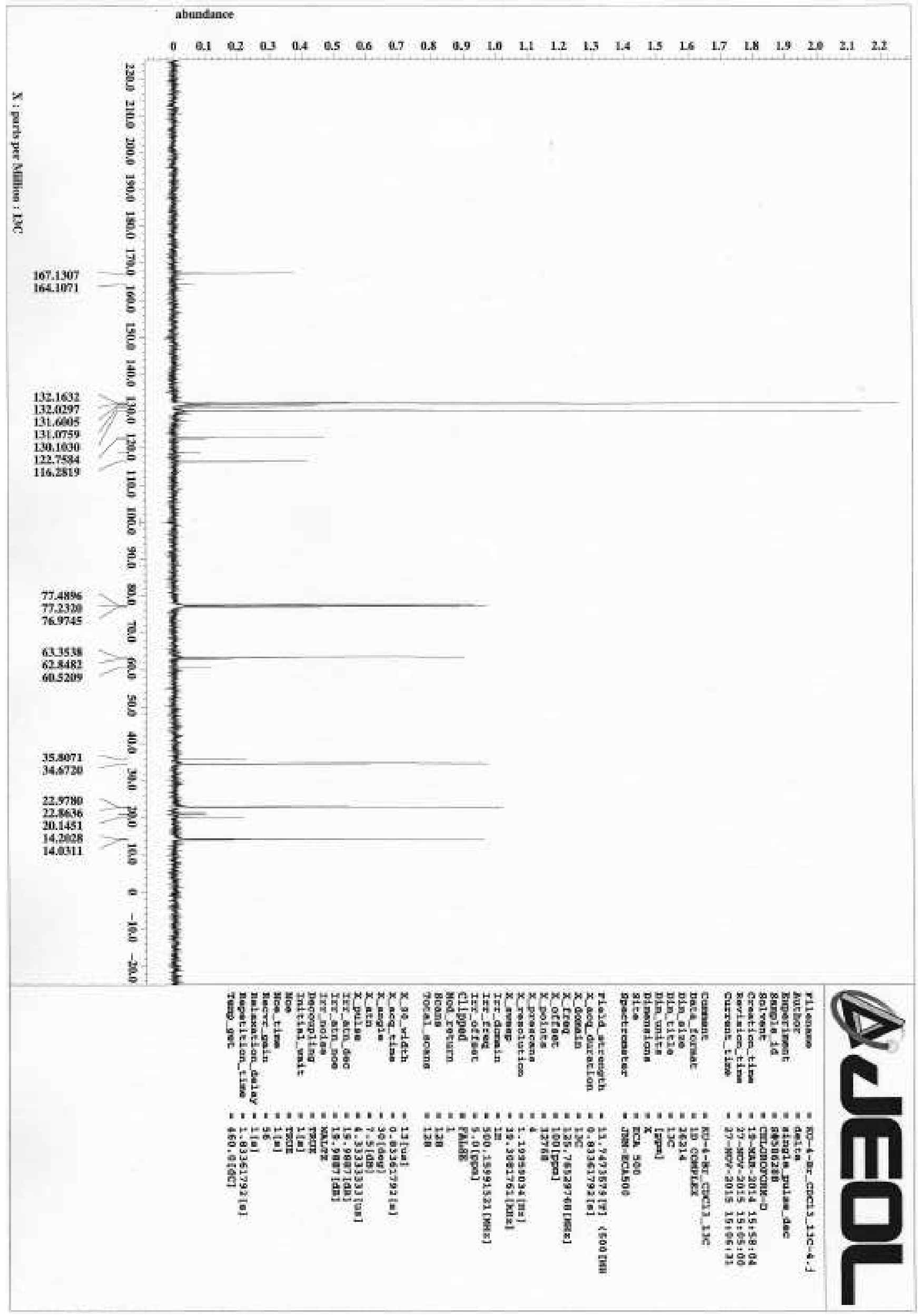


Ethyl 1-cyano-2-(4-nitrophenyl)cyclopropanecarboxylate (3i)<smiles>CCOC(=O)C1(C#N)CC1c1ccc([N+](=O)[O-])cc1</smiles>

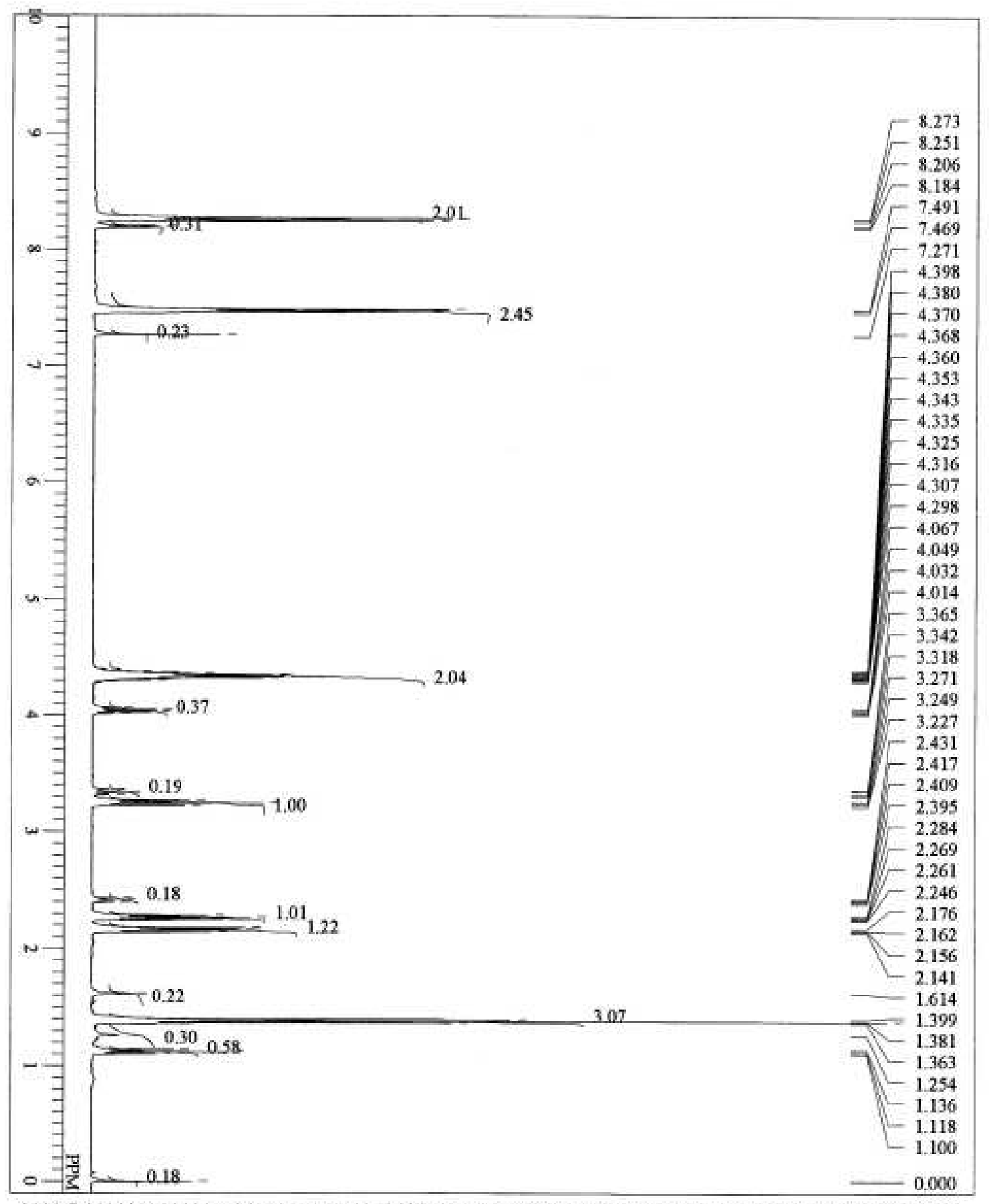

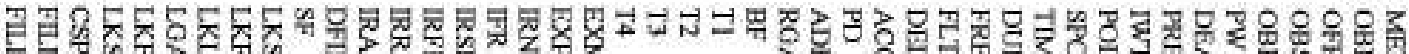

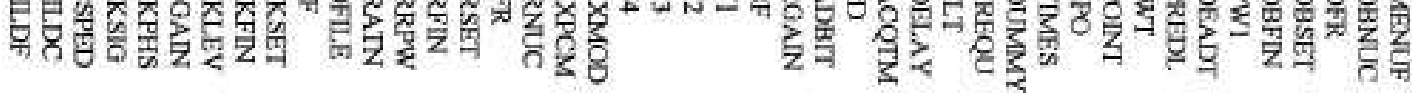

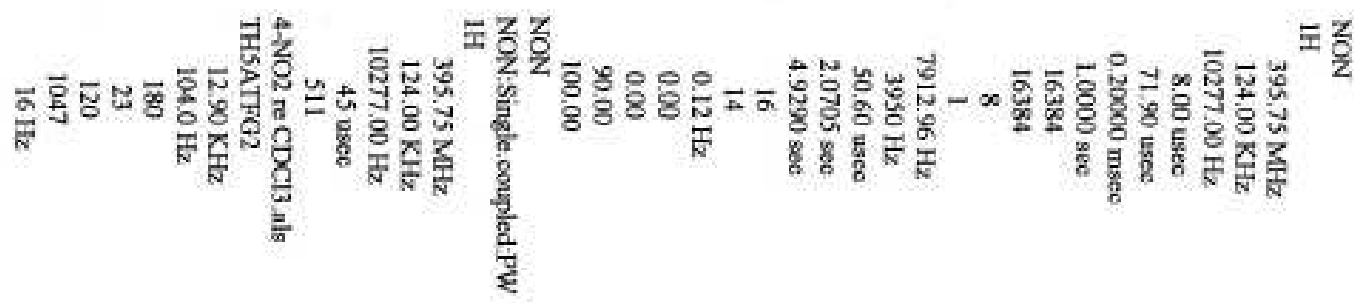




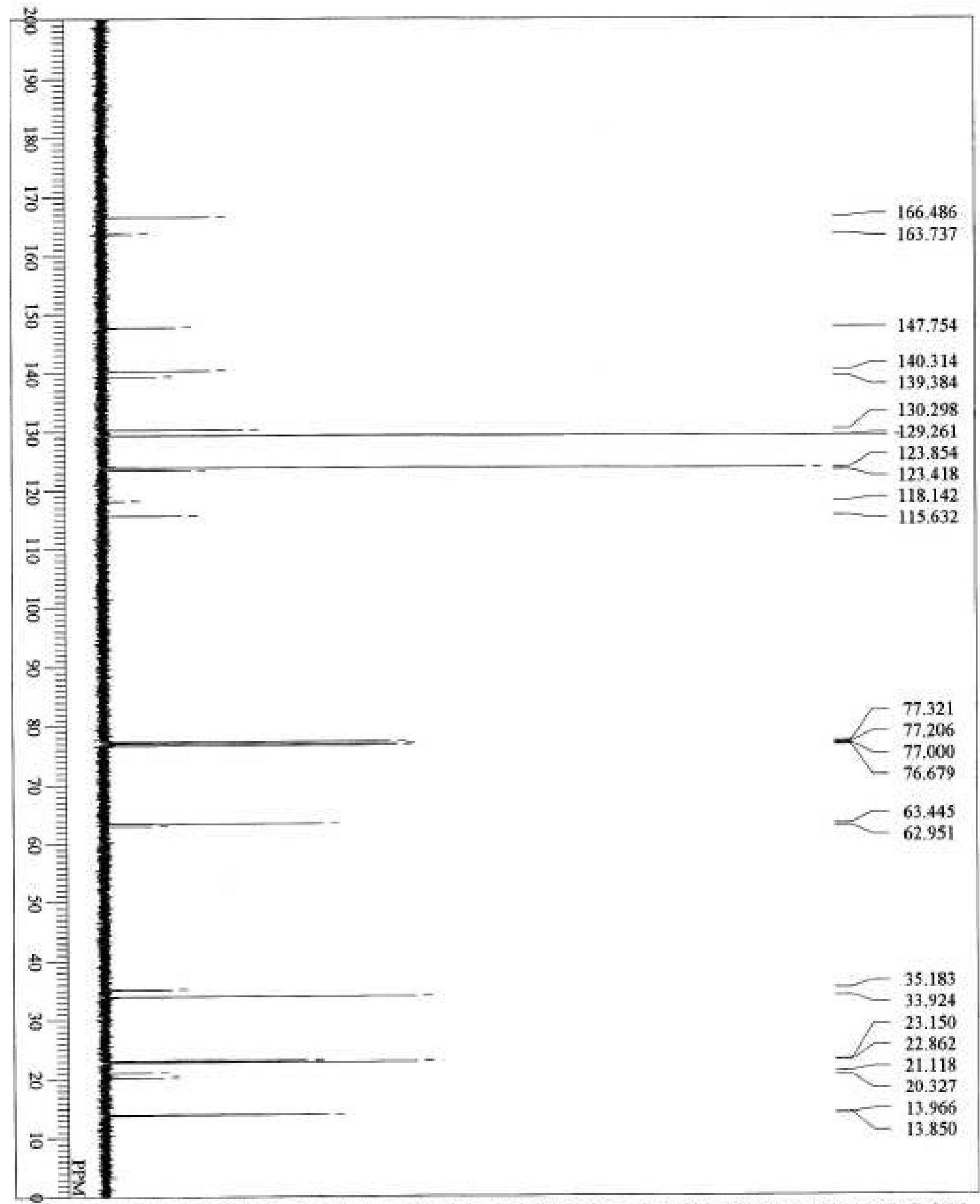

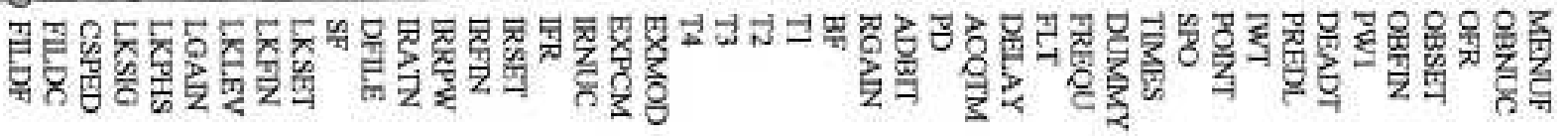

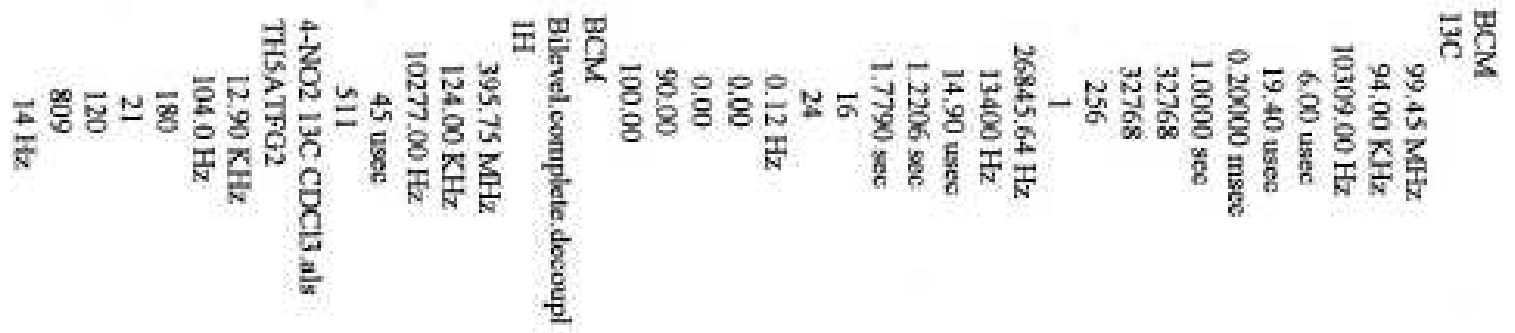


Ethyl 1-cyano-2-(2-pyridyl)cyclopropanecarboxylate (3j)<smiles>CCOC(=O)C1(C#N)CC1c1ccccn1</smiles>

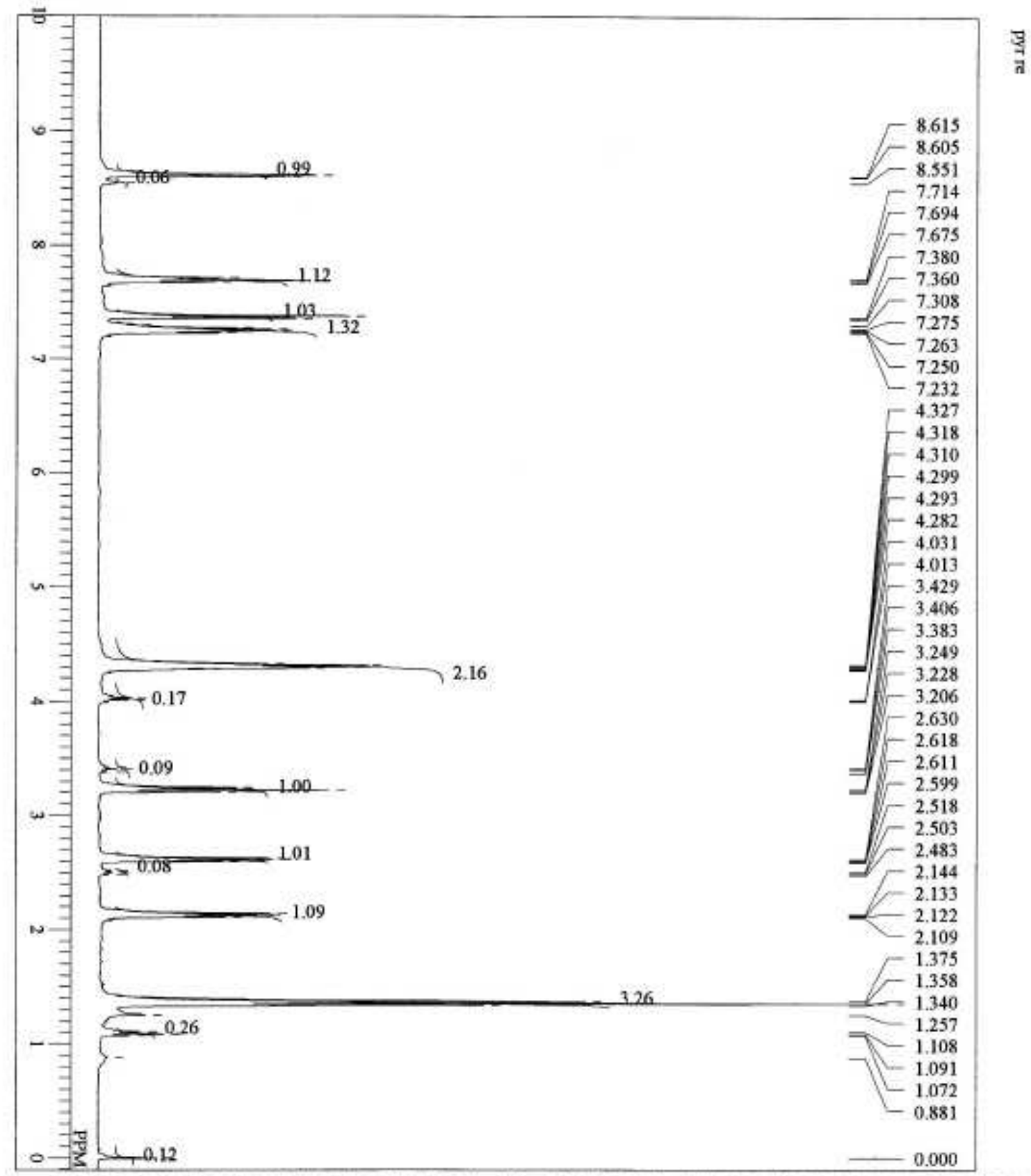

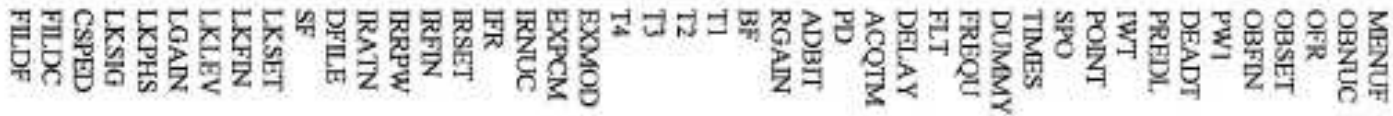

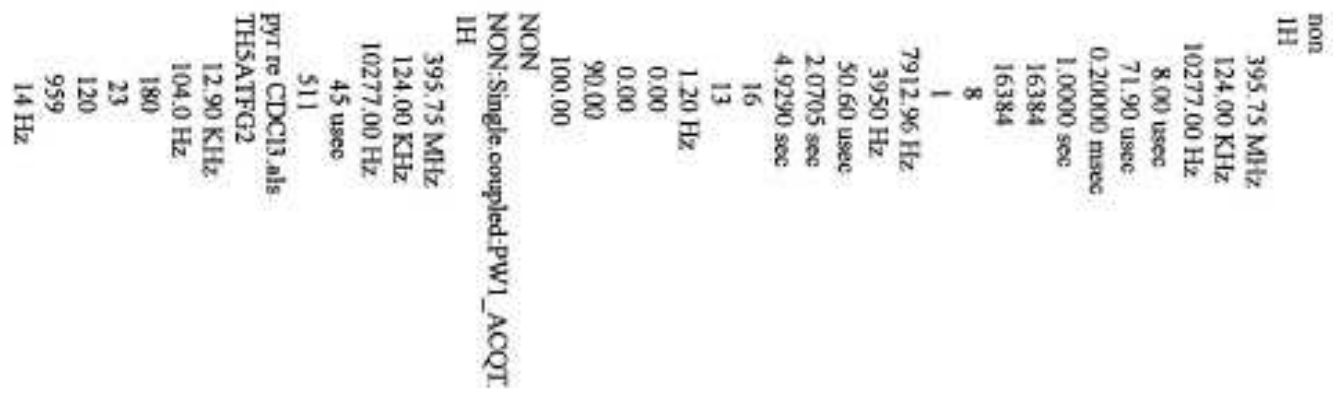




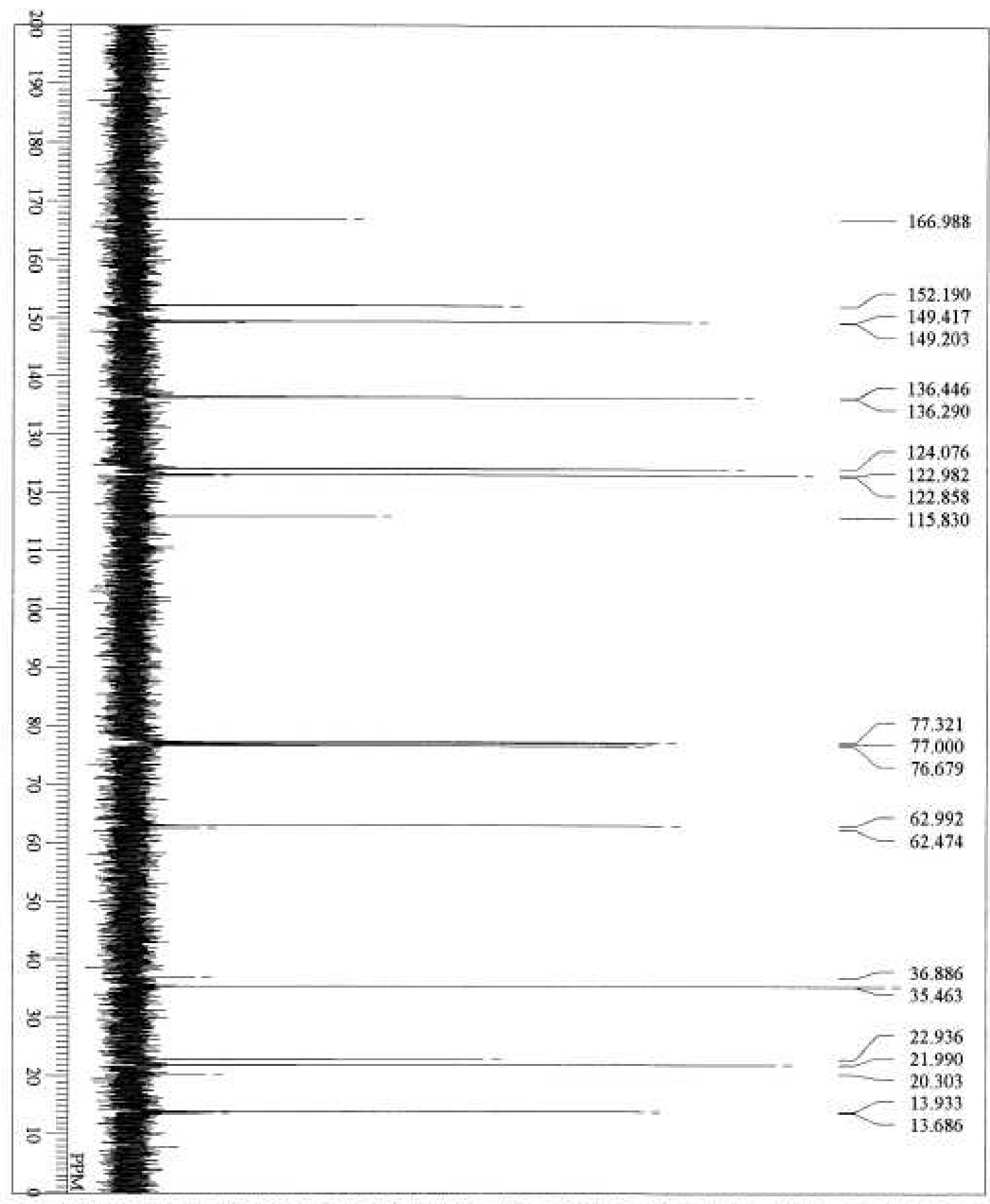

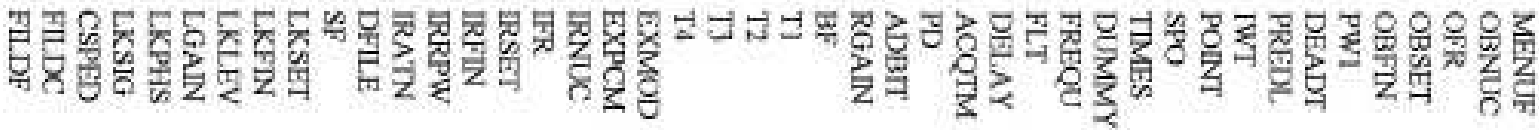

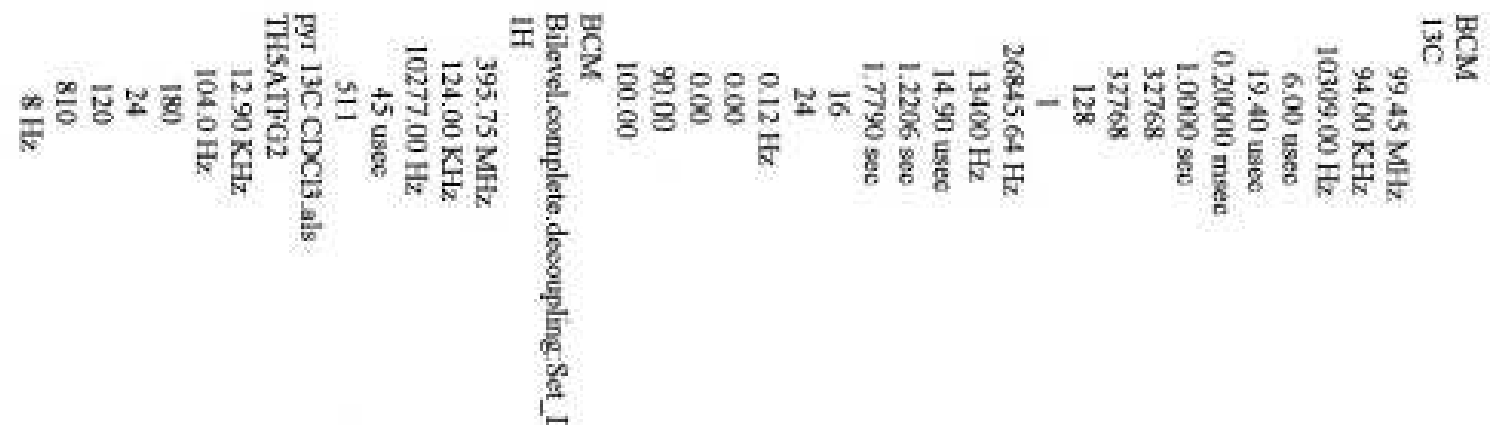


Ethyl 1-cyano-2-methyl-2-phenylcyclopropanecarboxylate (3k)<smiles>CCOC(=O)C1(C)OC1(C)c1ccccc1</smiles>

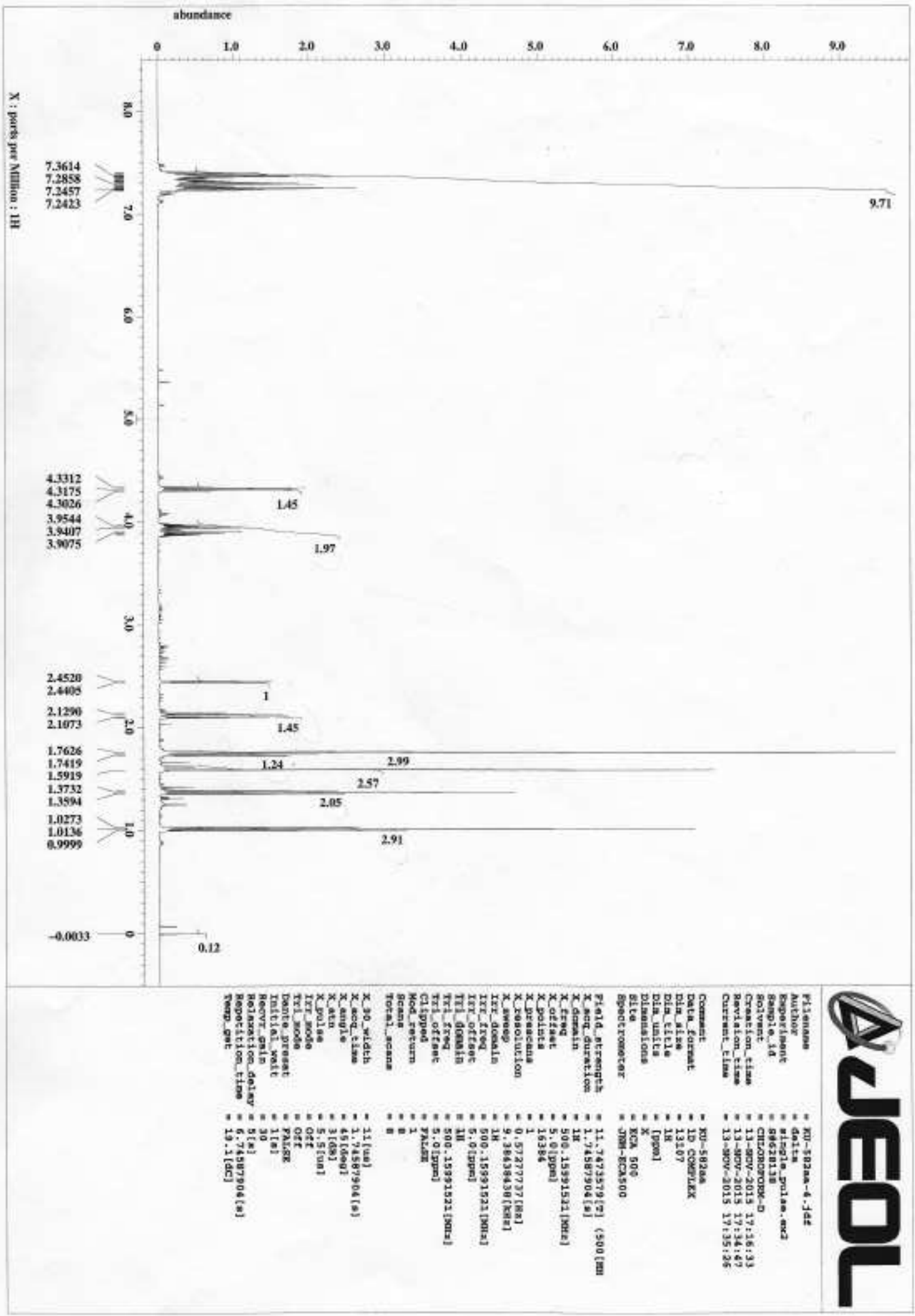




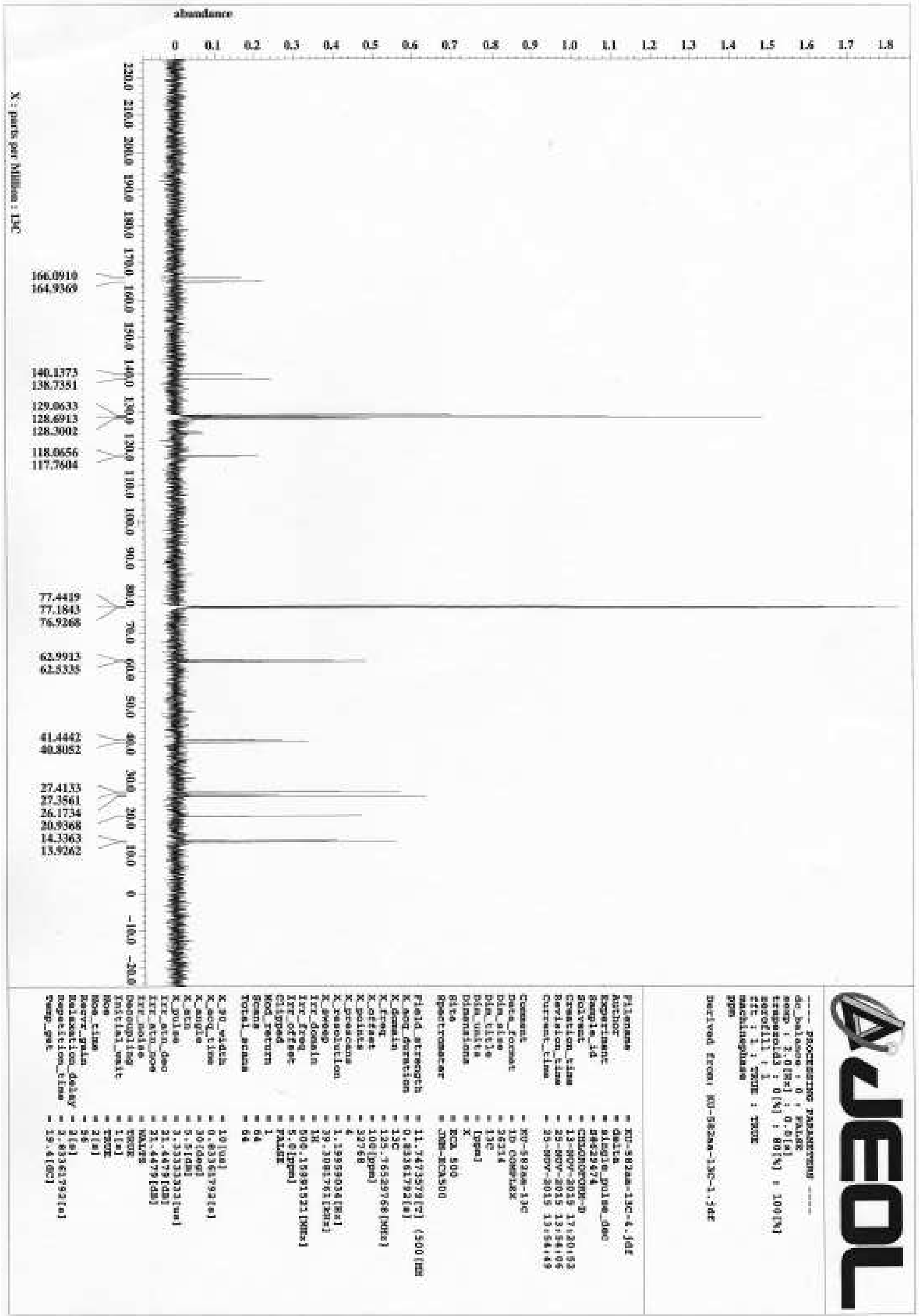


Ethyl 1-cyano-2-methyl-3-phenylcyclopropanecarboxylate (3l)<smiles>CCOC(=O)C(C)(C#N)C(C)c1ccccc1</smiles>

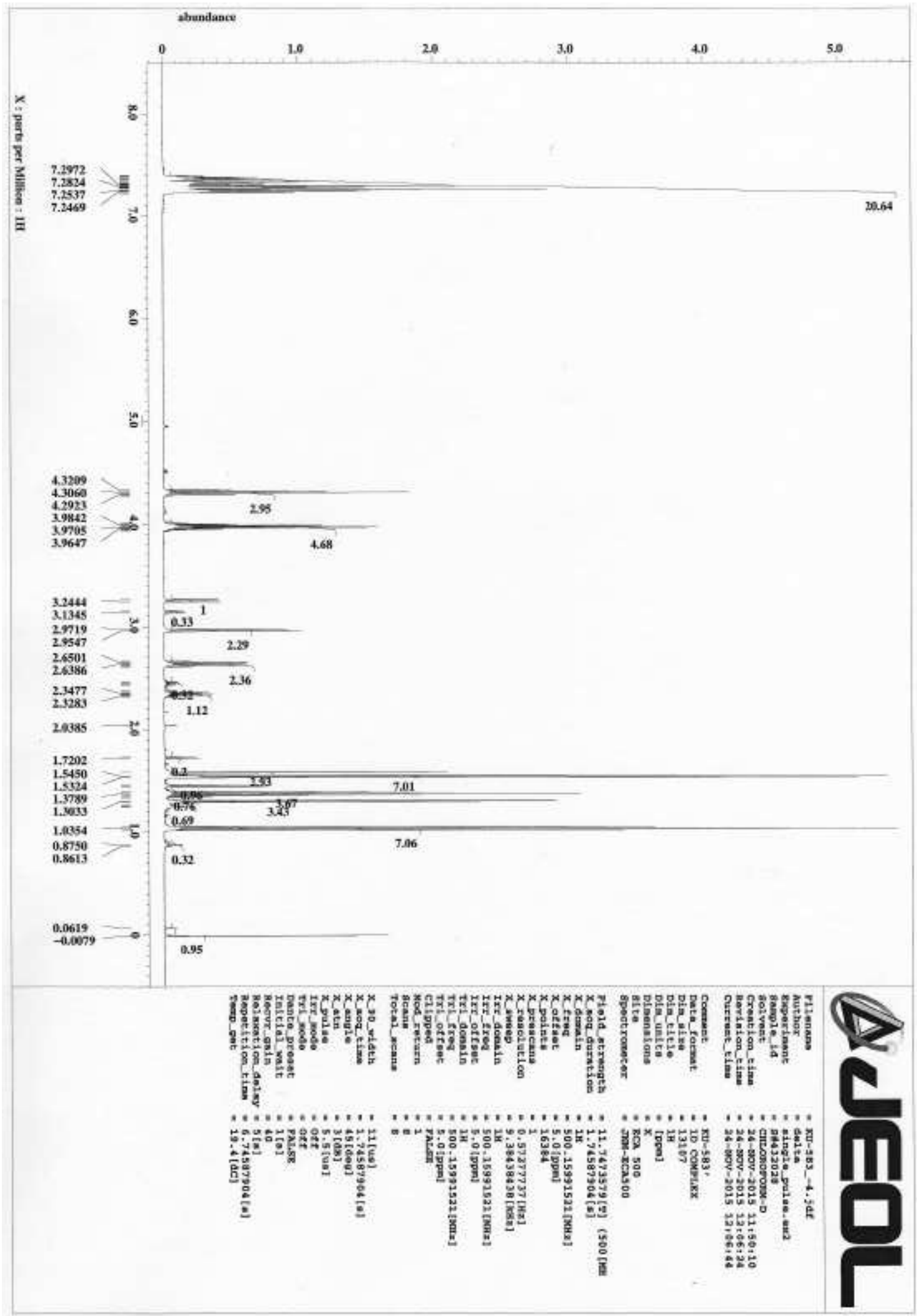




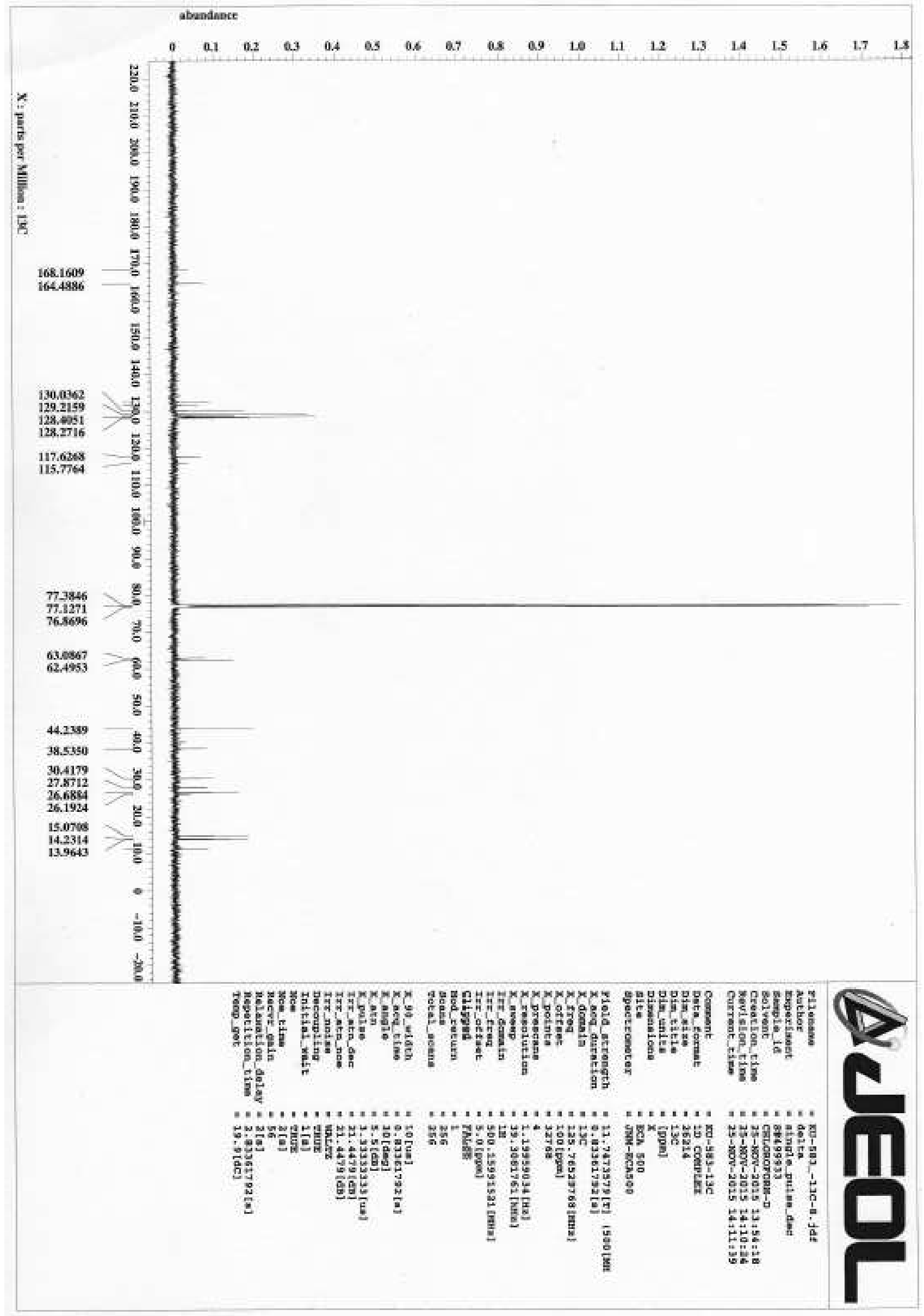


Ethyl 1-cyano-2,3-diphenylcyclopropanecarboxylate (3m)<smiles>CCOC(=O)C1(C#N)CC(c2ccccc2)CC1(C#N)c1ccccc1</smiles>

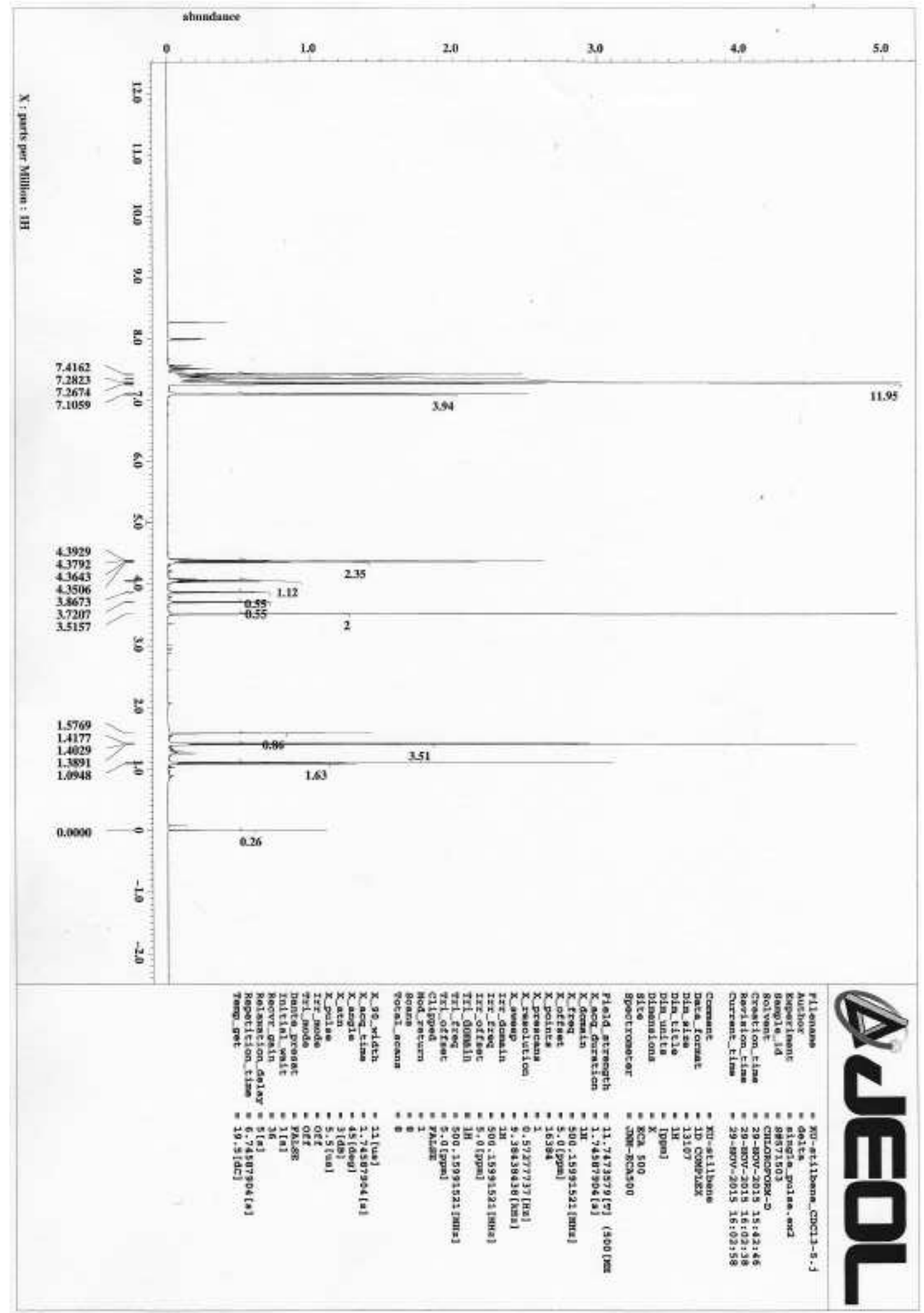




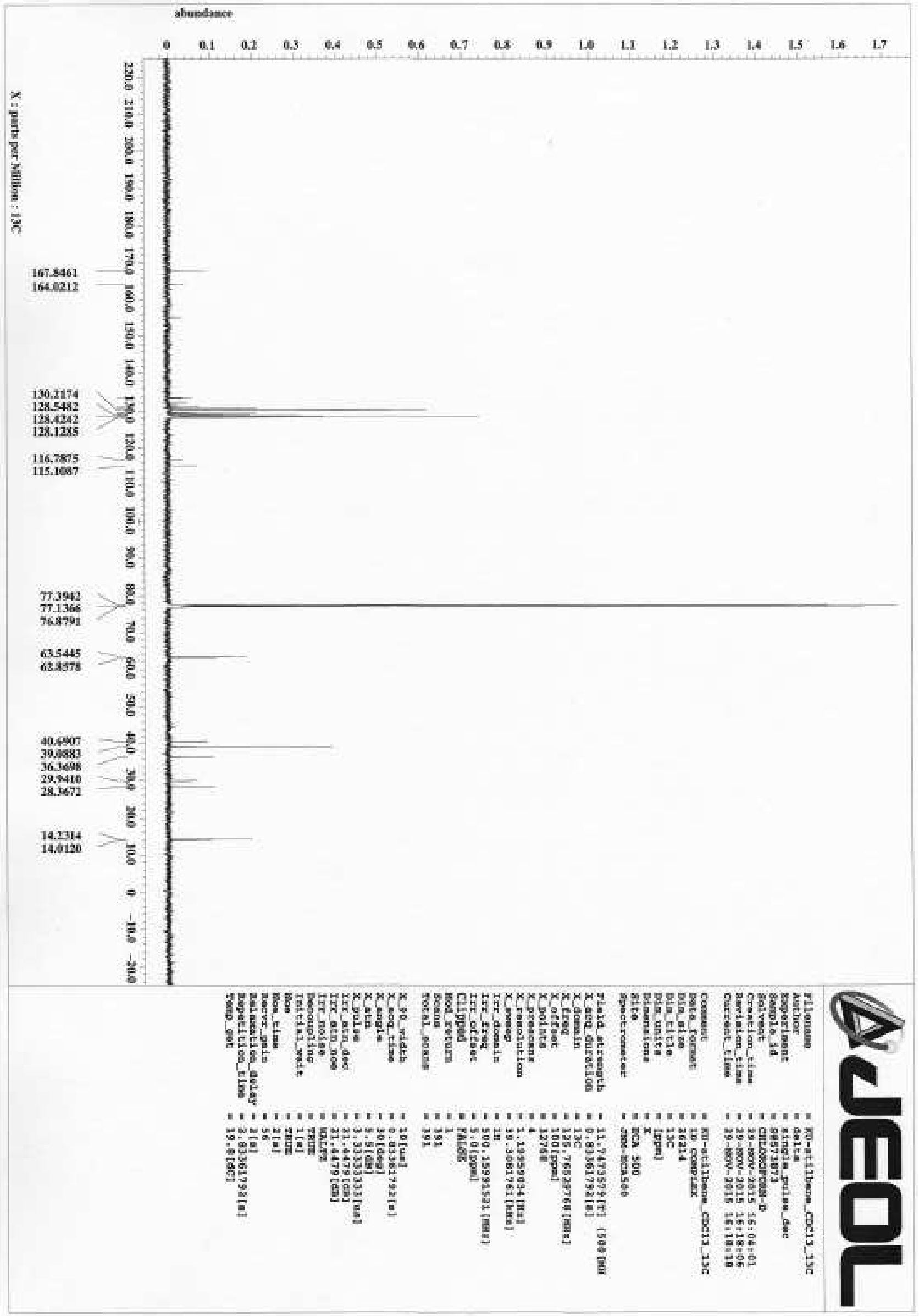


Methyl 1-cyano-2-phenylcyclopropanecarboxylate (3o)<smiles>COC(=O)C1(C#N)CC1c1ccccc1</smiles>
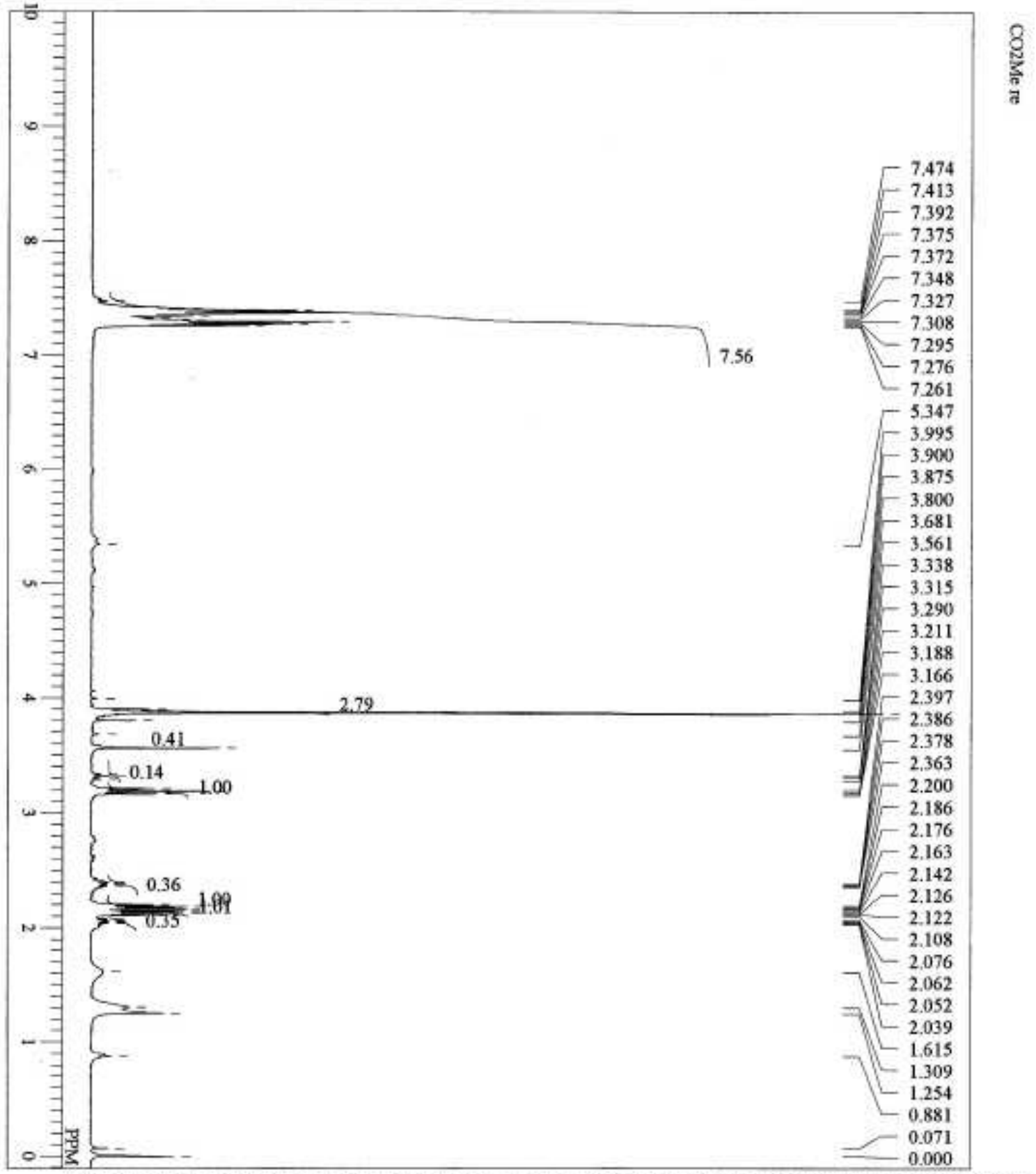

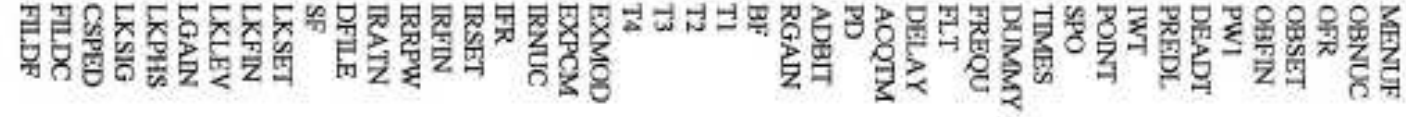

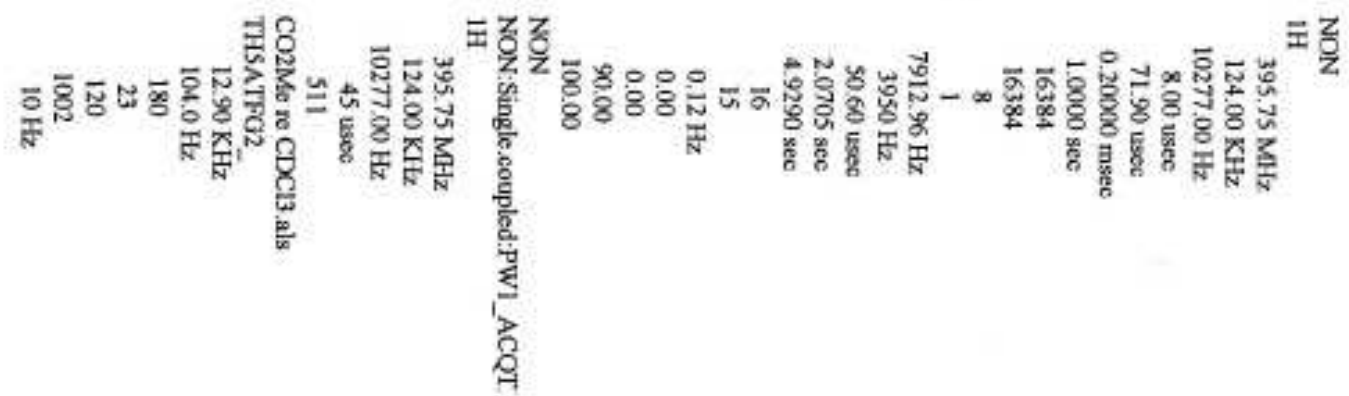




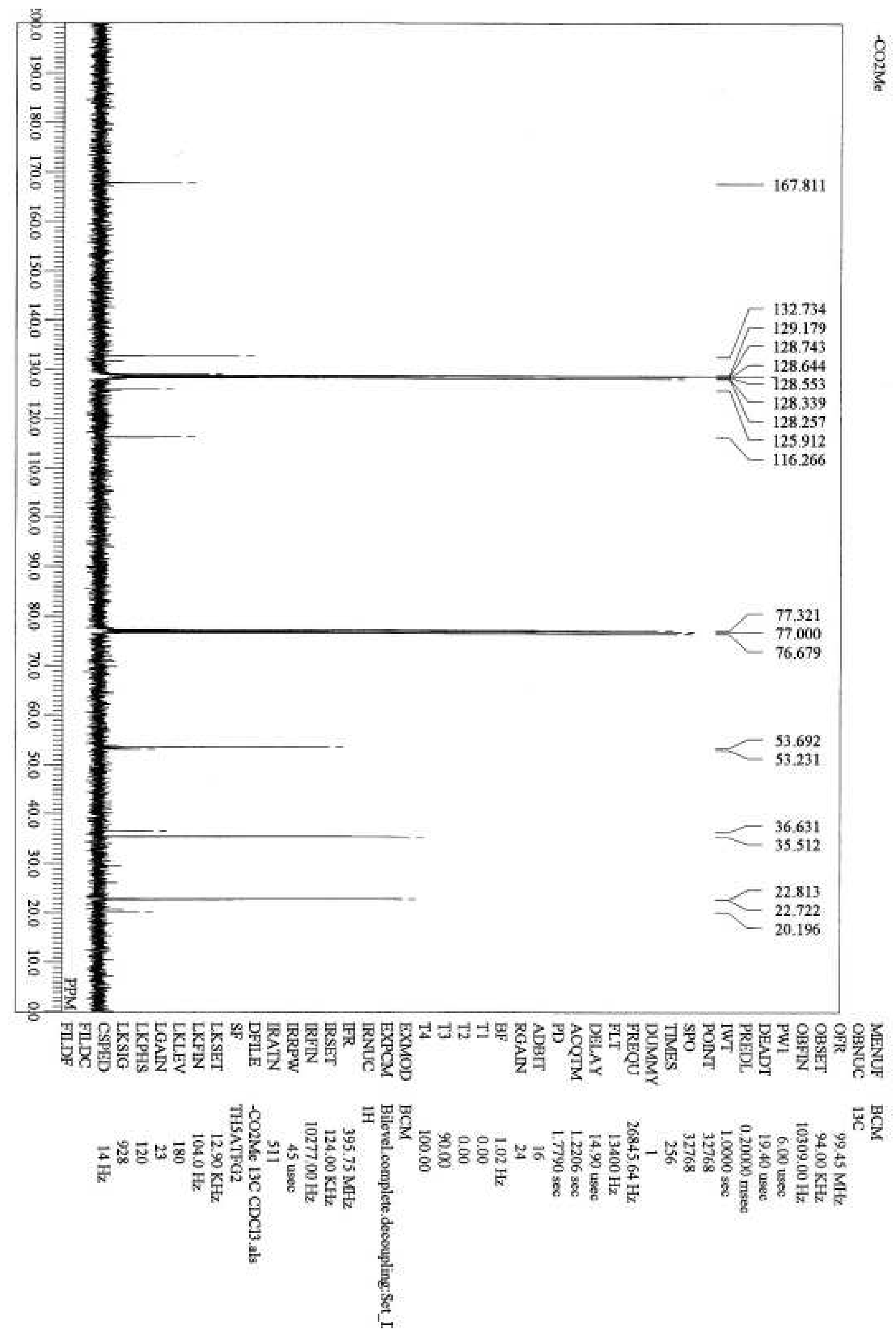




\section{1-Cyano-2-phenylcyclopropane-2-furyl ketone (3p)}<smiles>CC1(C(=O)c2ccco2)CC1c1ccccc1</smiles>

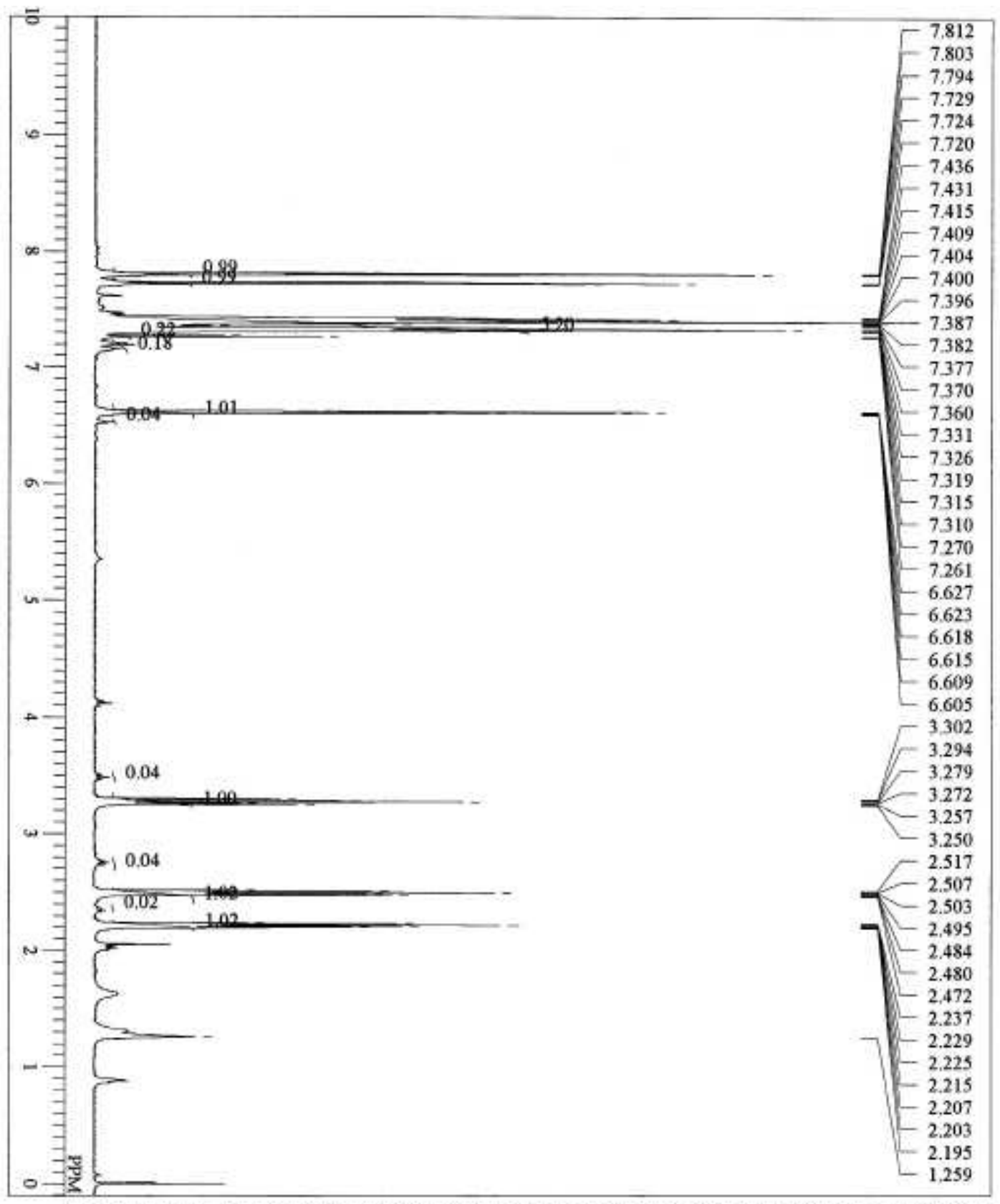

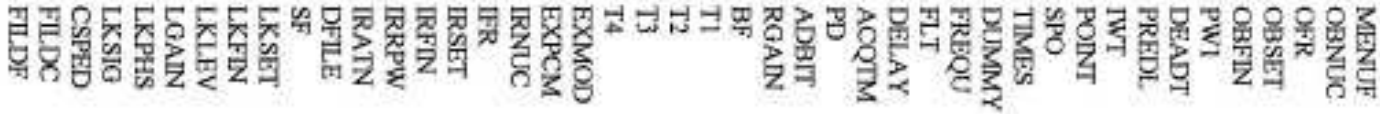

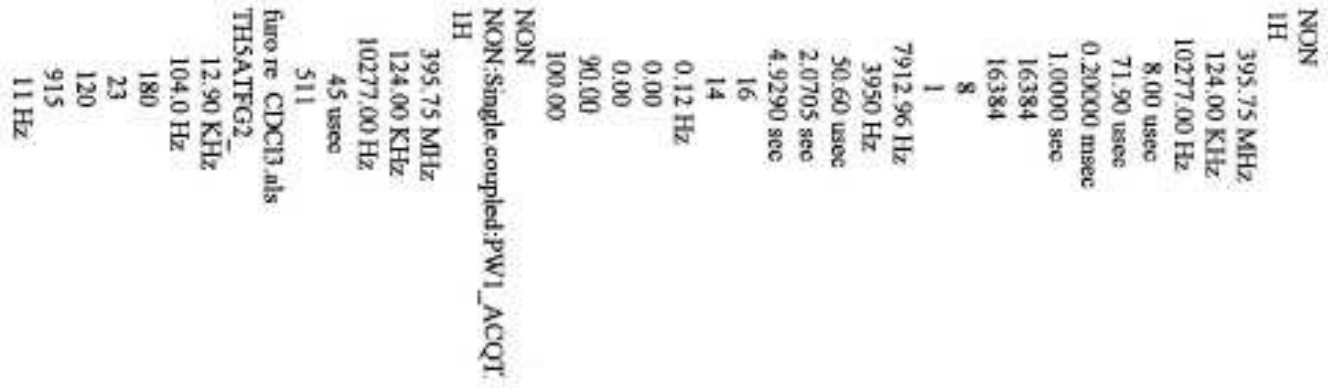




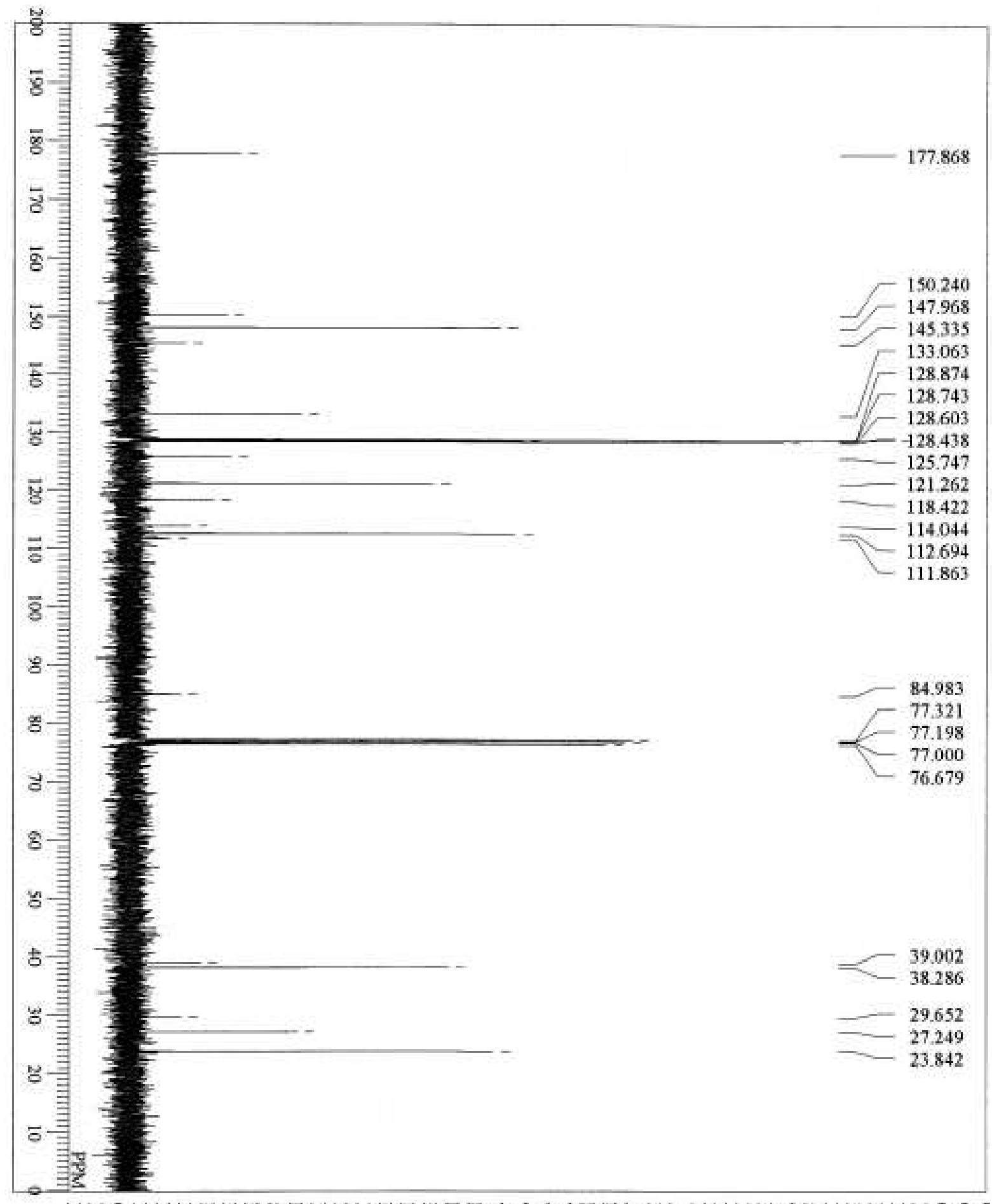

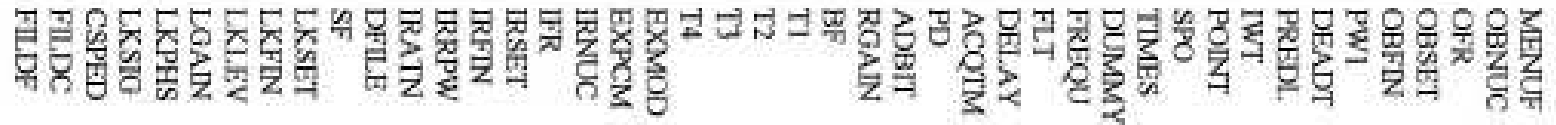

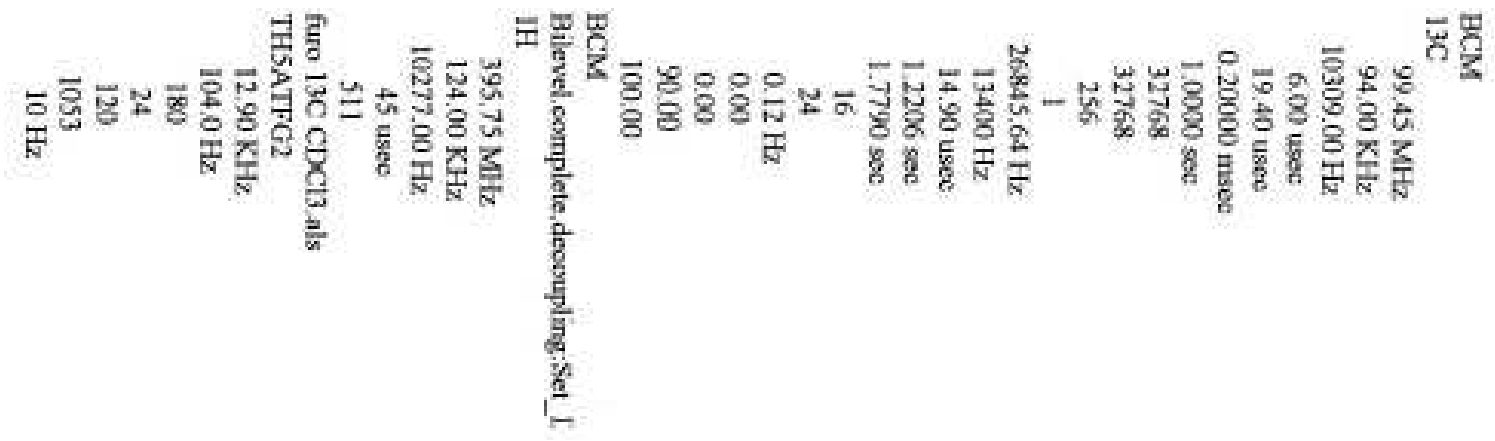


1-Cyano-2-phenylcyclopropane-2-toluoyl ketone (3q)<smiles>Cc1ccc(C(=O)C2(C#N)CC2c2ccccc2)cc1</smiles>

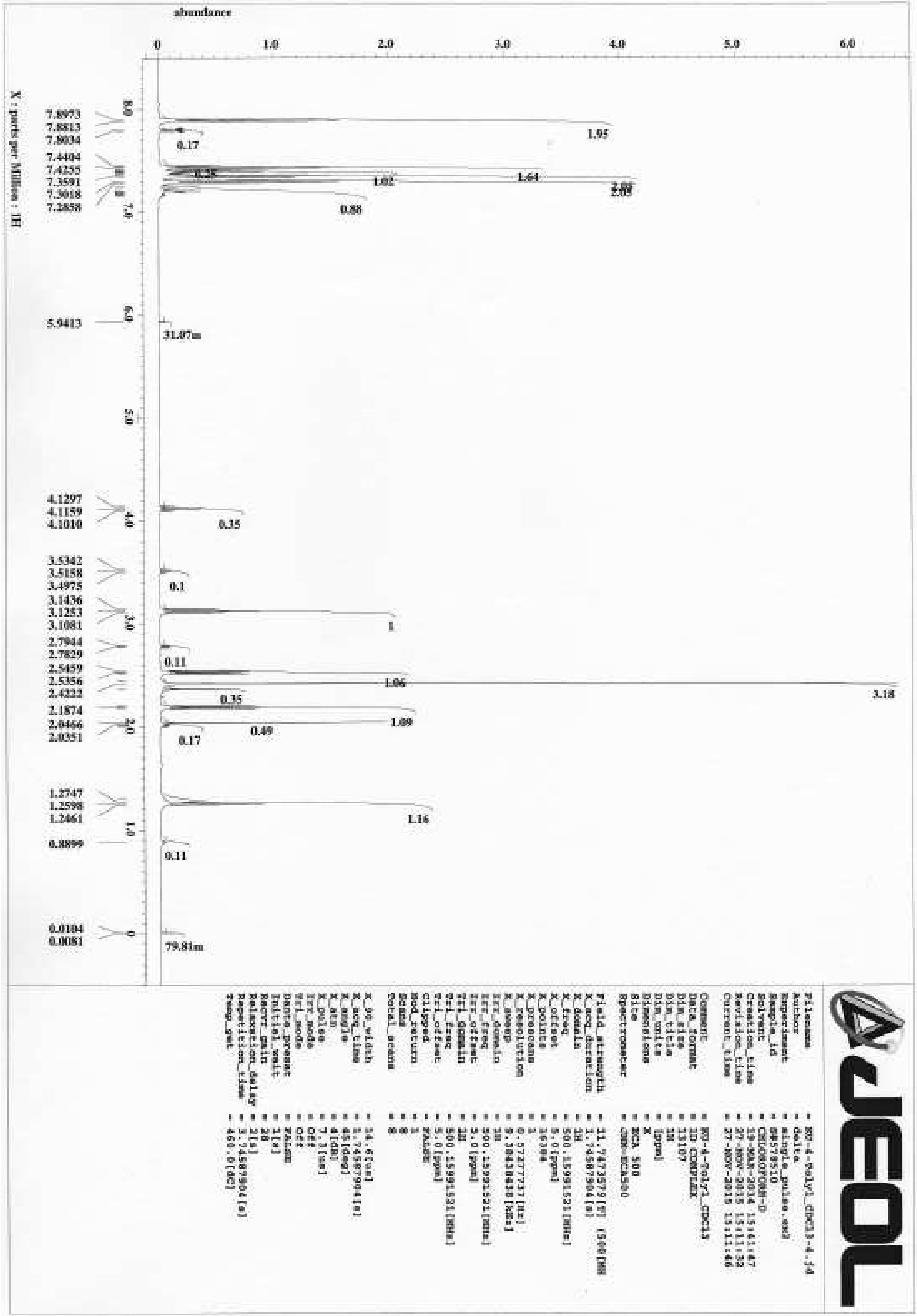




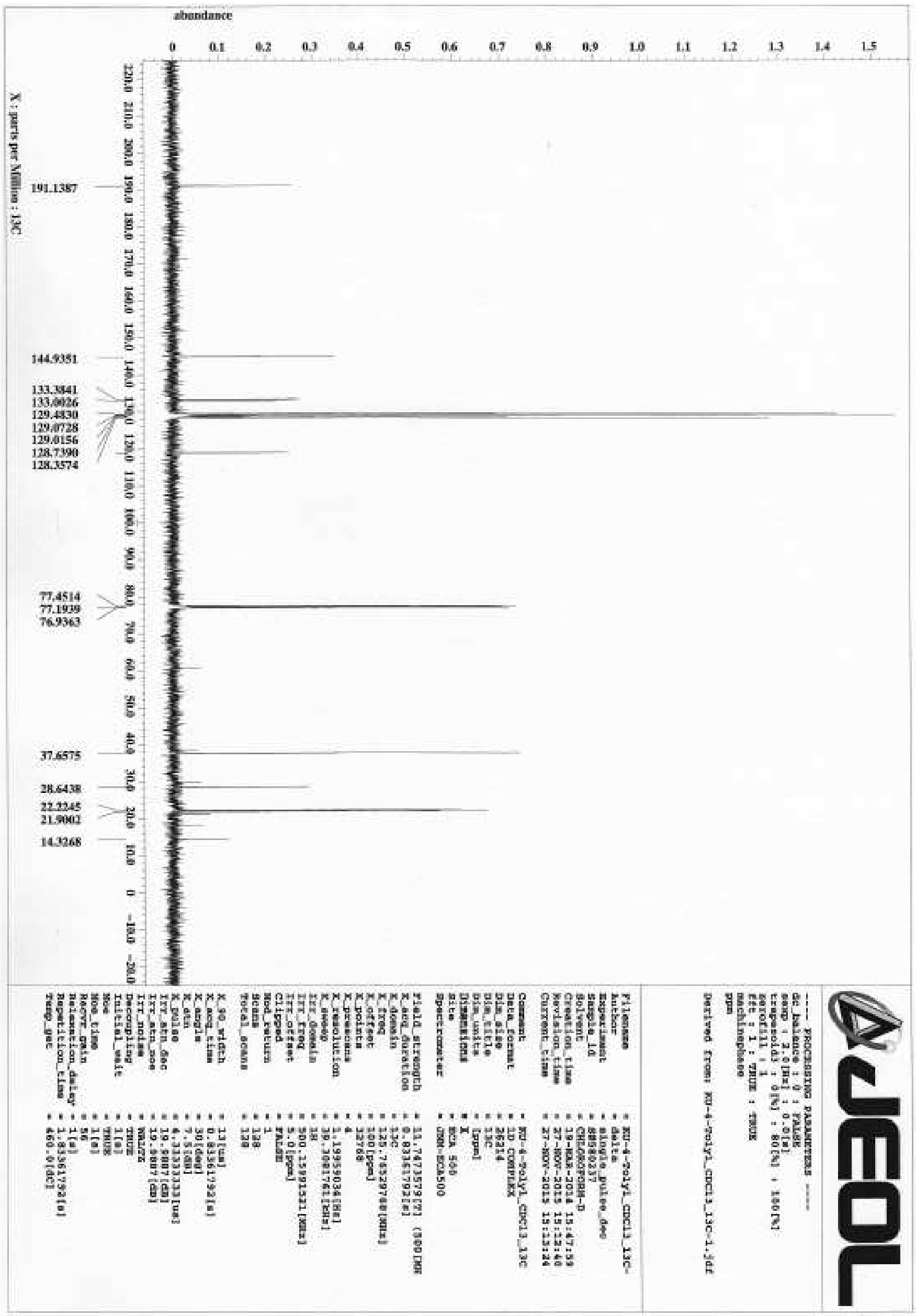




\section{1-Cyano-2-phenylcyclopropane-2-pivaloyl ketone (3r)}<smiles>CC(C)(C)C(=O)C1(C#N)CC1c1ccccc1</smiles>

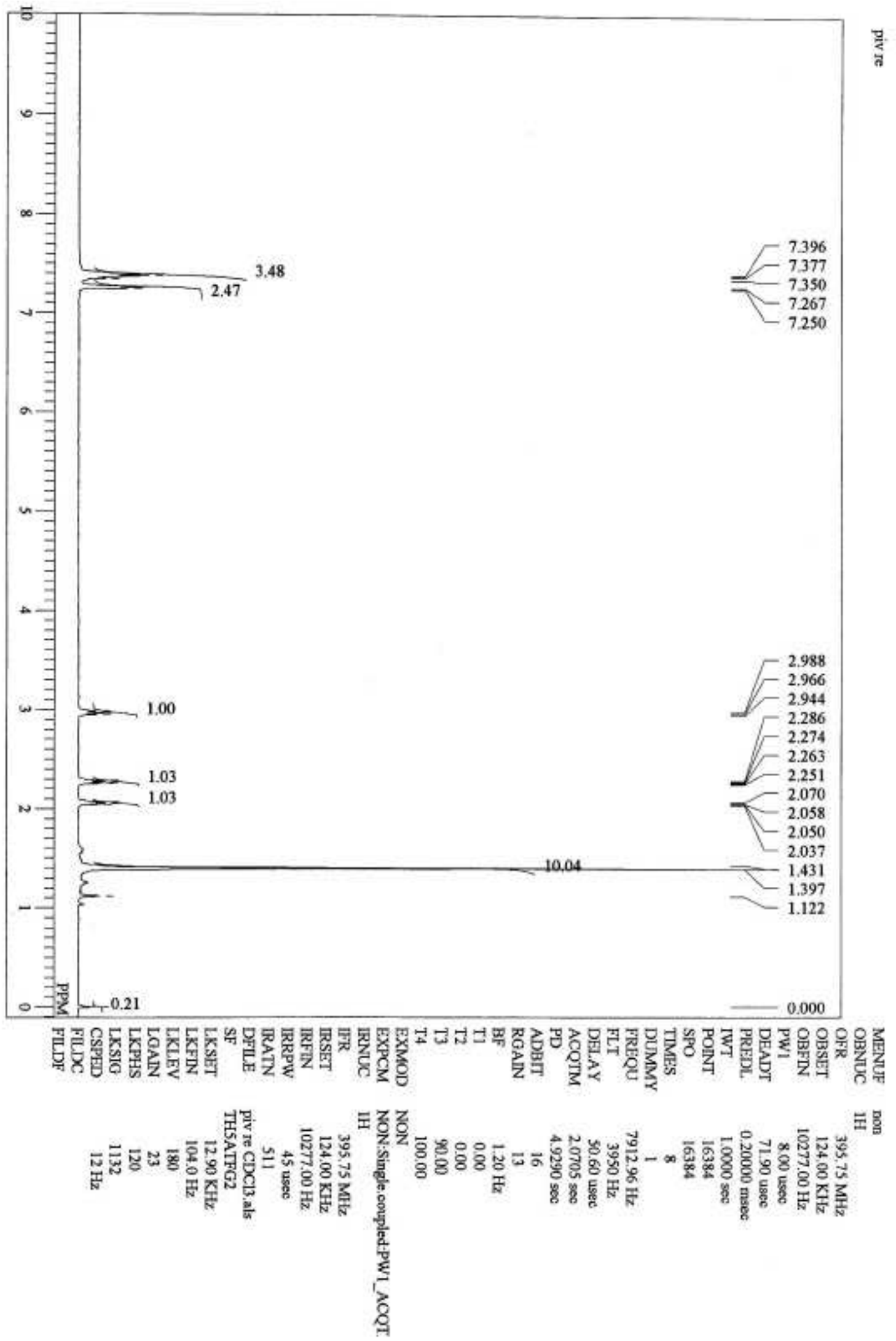




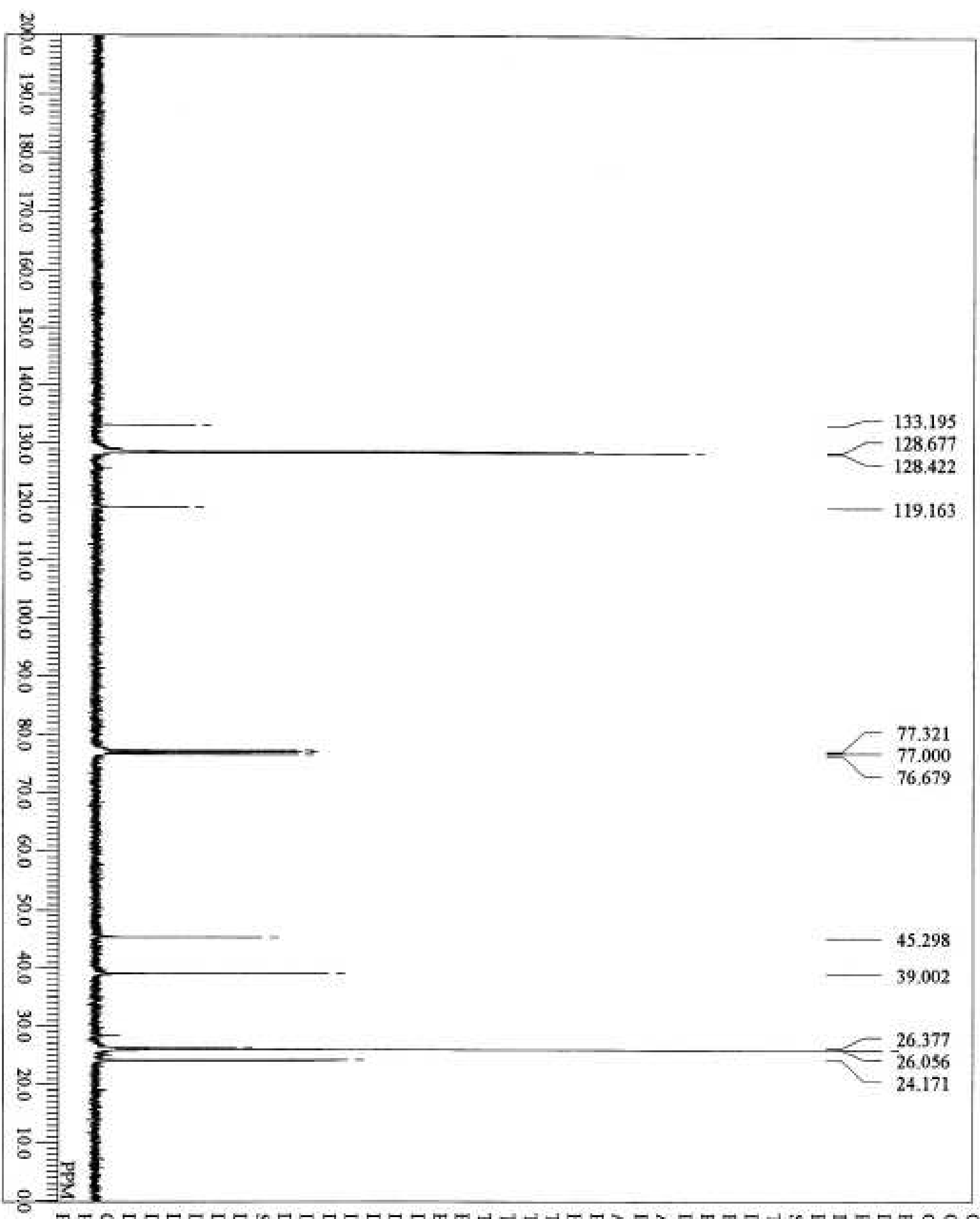

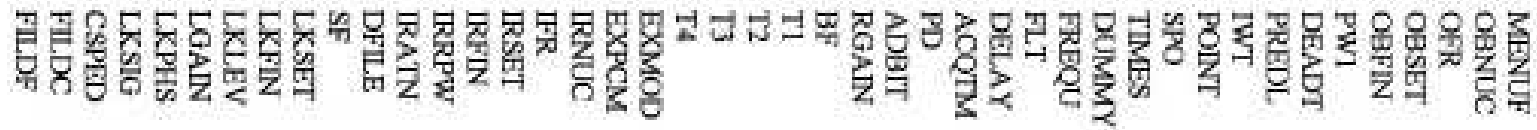

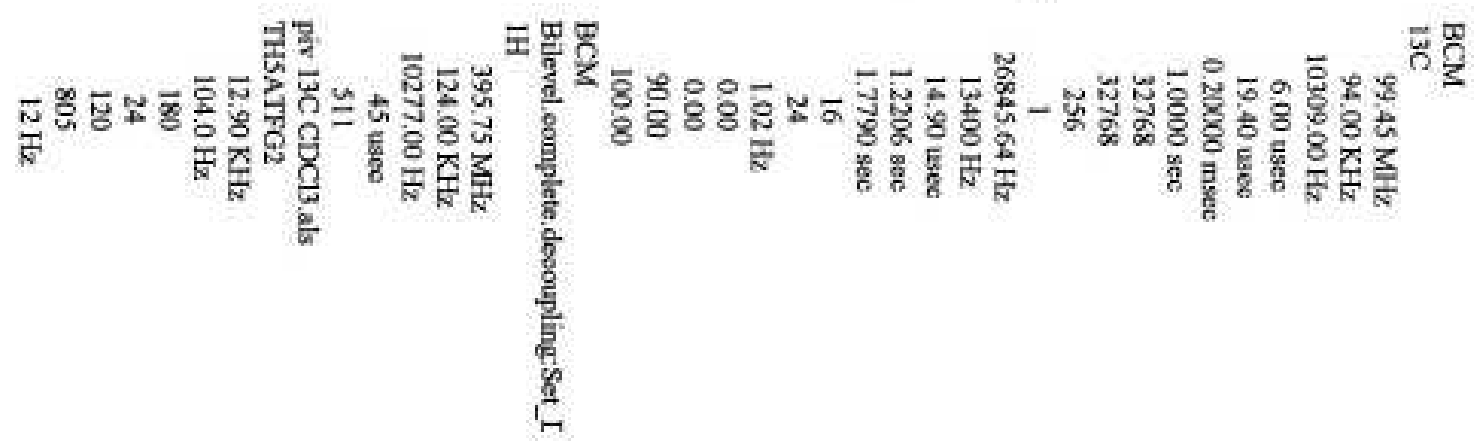


1,1-Dicyano-2-phenylcyclopropane (3s)<smiles>N#CC1(C#N)CC1c1ccccc1</smiles>

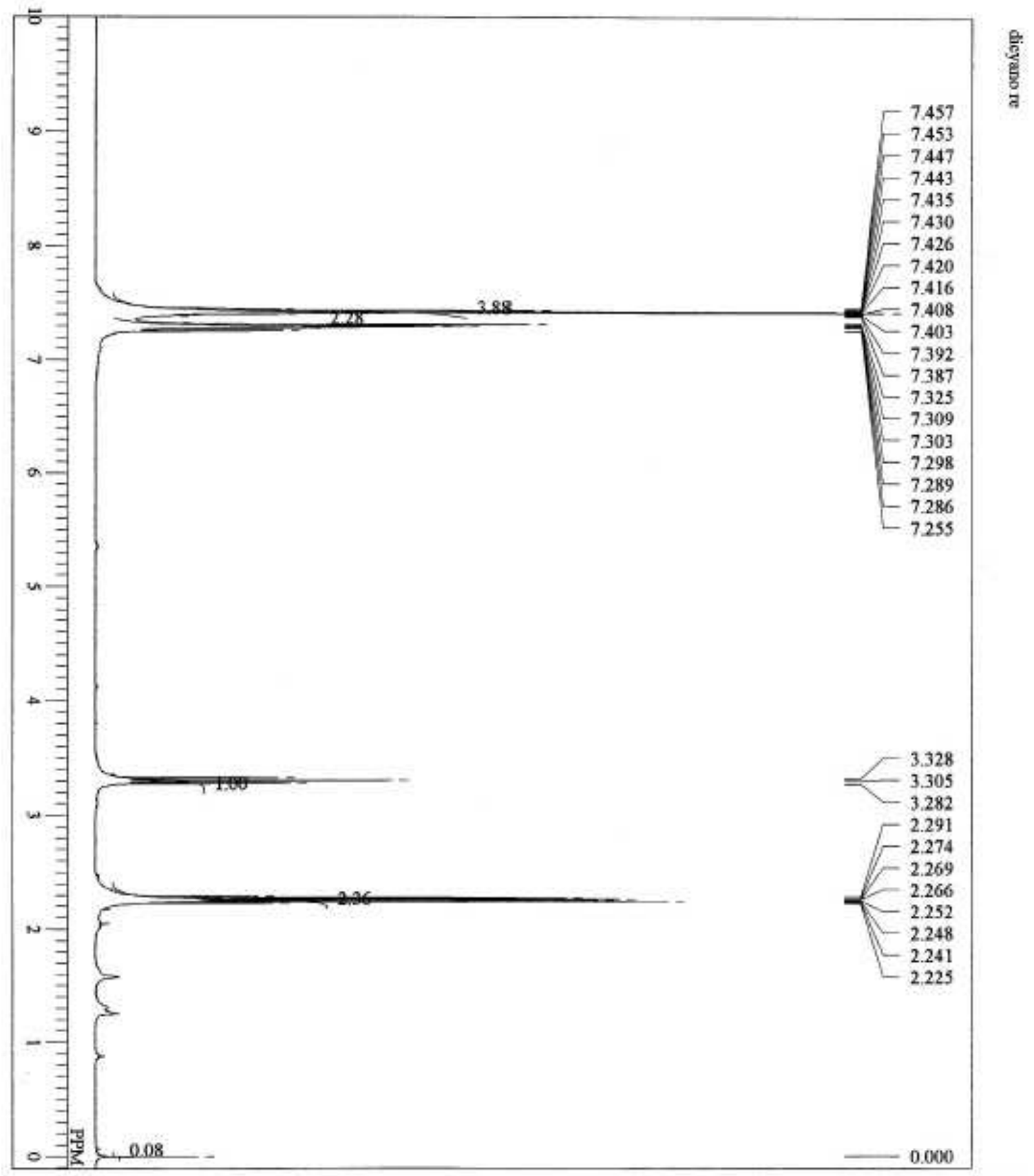

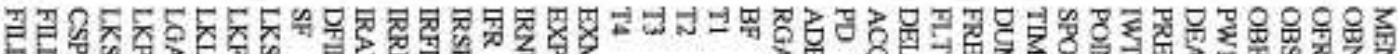

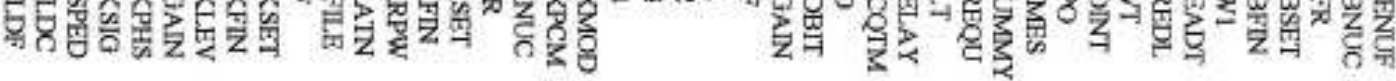

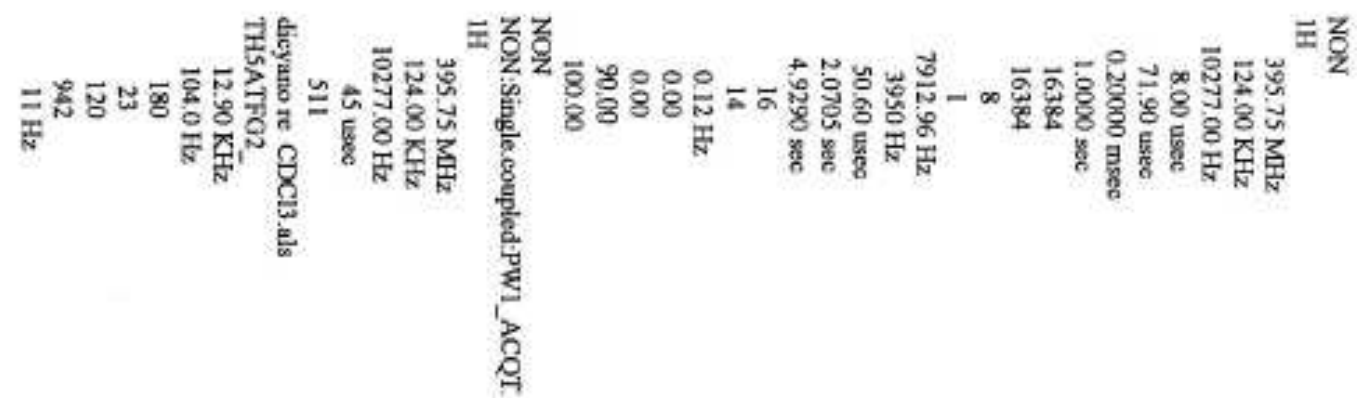




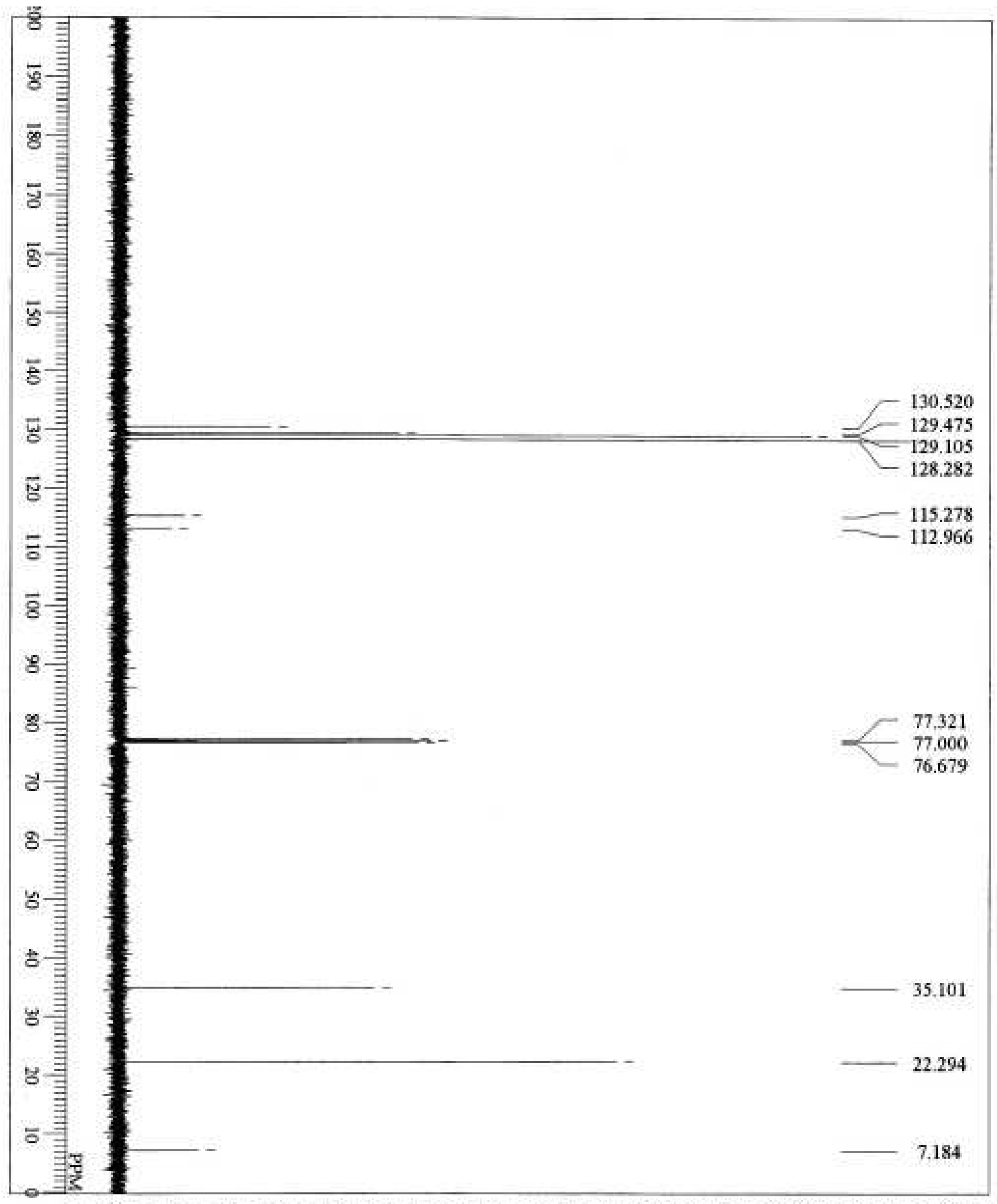

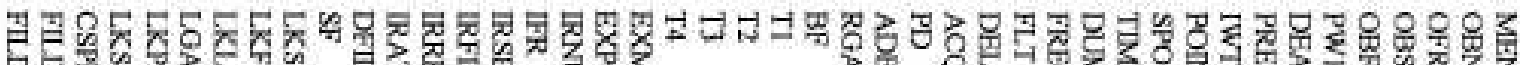

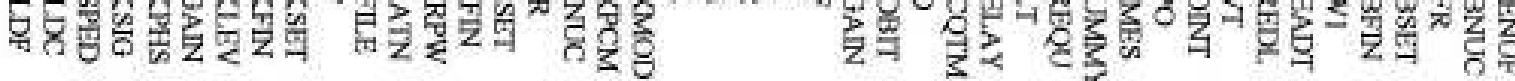

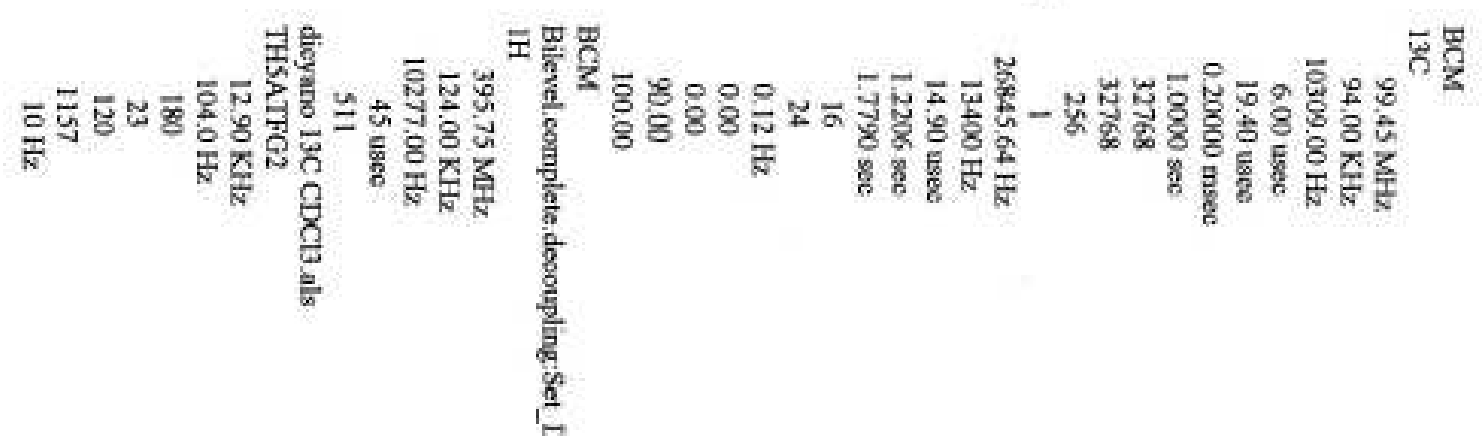


1-Phenyl-1,1-cyclopropanedicarboxylic acid diethyl ester (3t)<smiles>CCOCC(C)(OCC)C1CC1c1ccccc1</smiles>

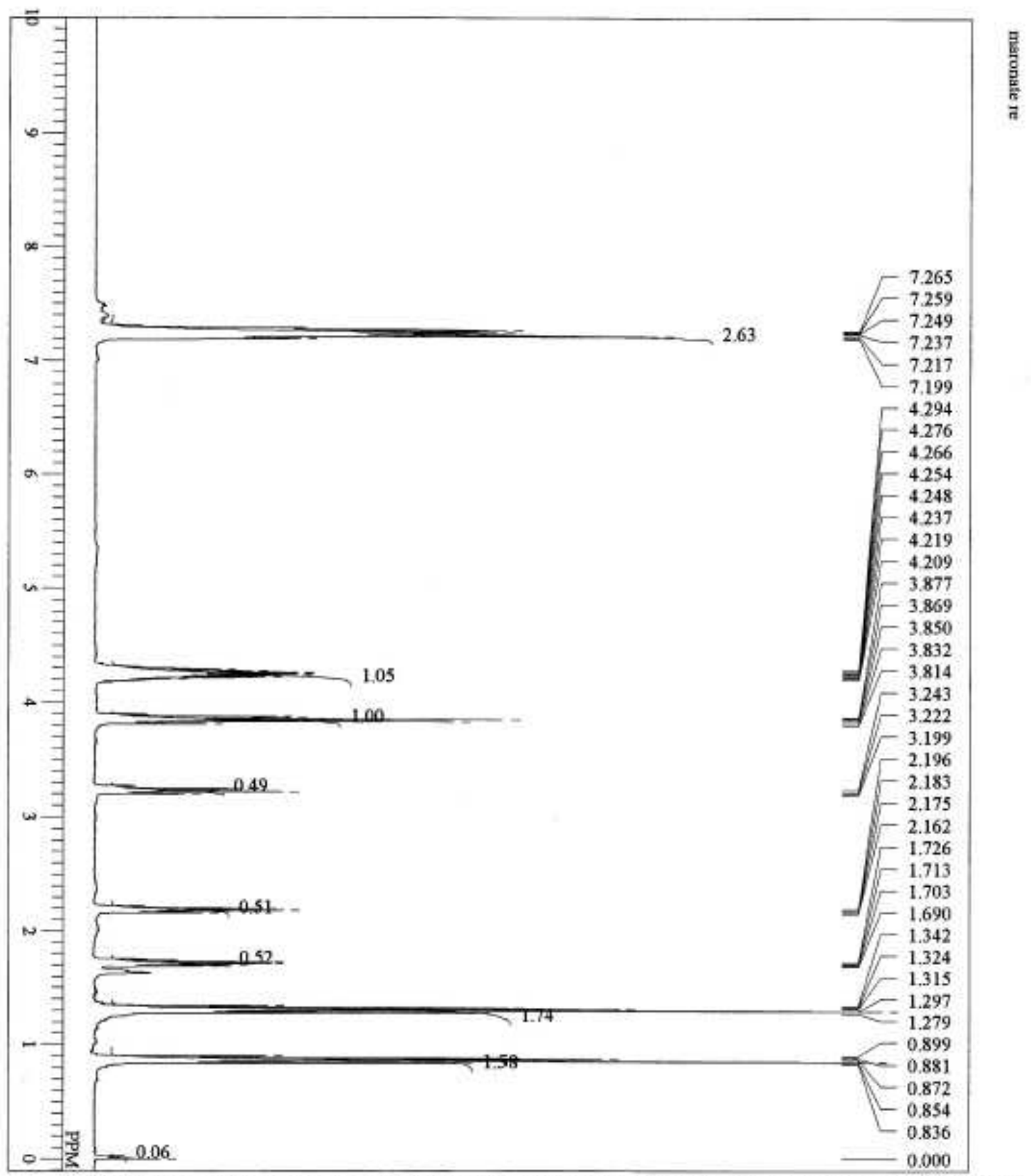

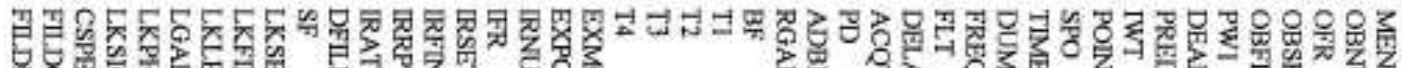

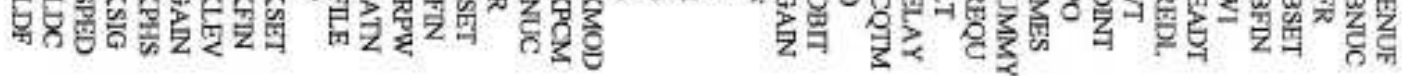

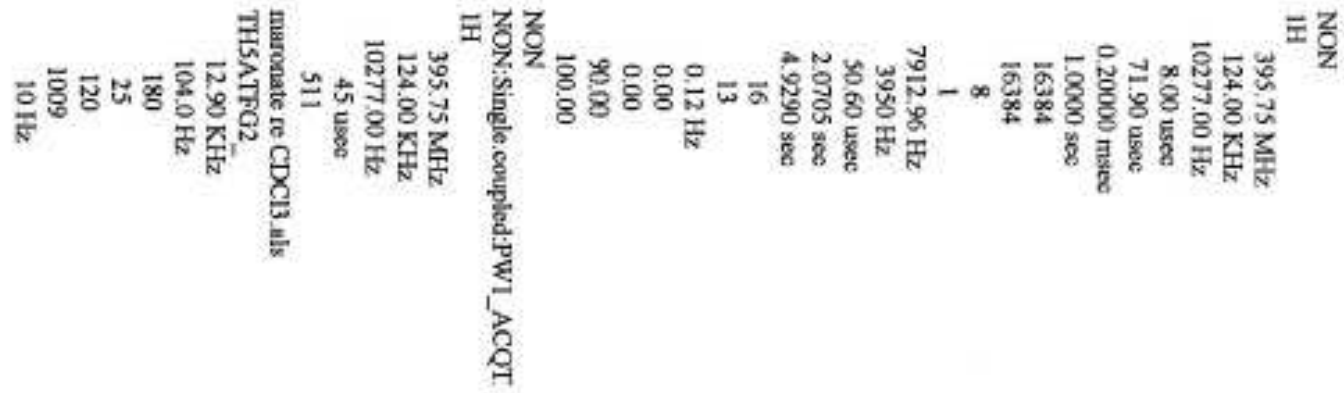




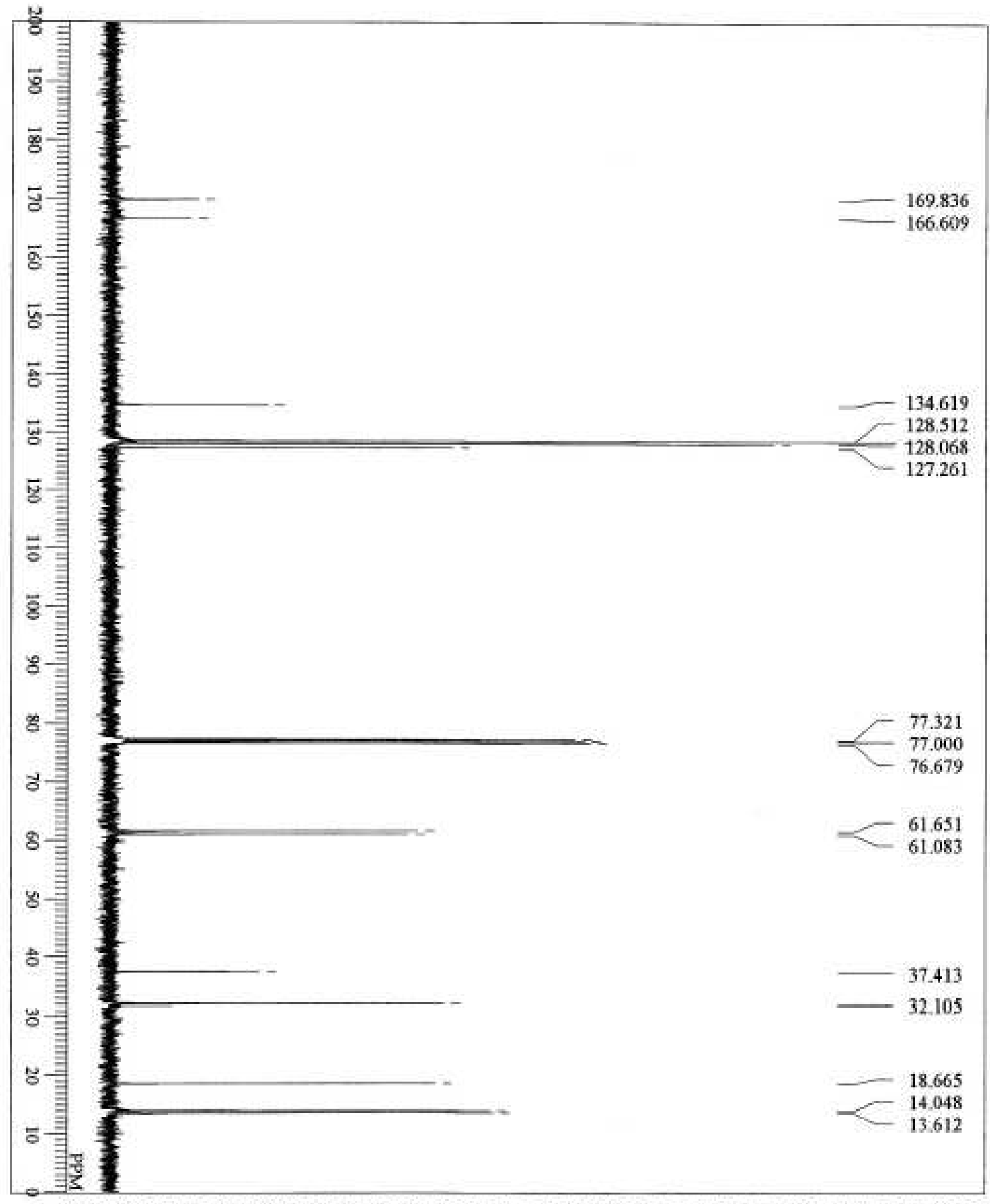

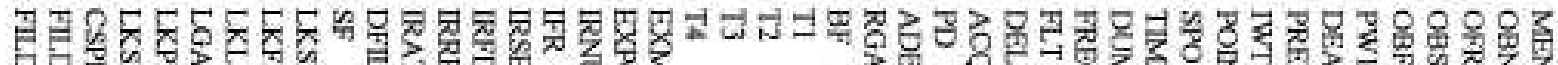

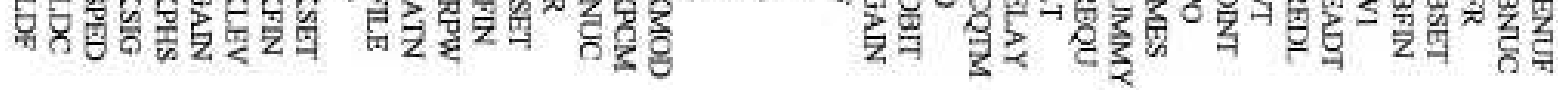

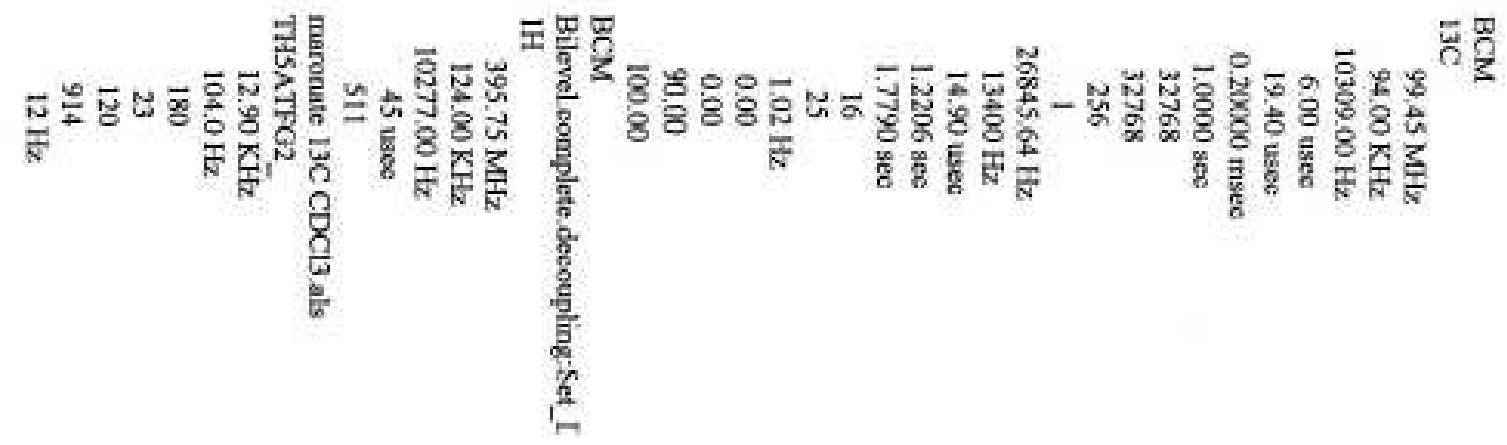

